\section{A Primer on the Conditional Mediation Analysis in PLS-SEM}

\author{
Jun-Hwa Cheah \\ Universiti Putra Malaysia
}

Christian Nitzl

Bundeswehr University Munich

José L. Roldán

Universidad de Sevilla

Gabriel Cepeda-Carrion

Universidad de Sevilla

Siegfried P. Gudergan

James Cook University

\section{Acknowledgments}

The authors would like to thank both Christian M. Ringle and Marko Sarstedt for suggesting us the idea on tackling the conditional mediation (CoMe) analysis in partial least squares structural equation modeling (PLS-SEM).

\begin{abstract}
Conditional mediation (CoMe) analysis integrates mediation and moderation analyses to examine and test hypotheses about how mediated relationships vary as a function of context, boundaries, or individual differences. Although CoMe analysis can be a crucial element of empirical studies that seek to advance theory in information systems, applications of such analysis are scarce, in general, and in partial least squares structural equation modeling (PLS-SEM), in particular. This paper clarifies conceptual fundamentals of and develops guidelines for CoMe analysis within the PLS-SEM context. Furthermore, the paper outlines the illustrative use of CoMe analysis in PLS-SEM and presents detailed step-by-step procedures to do so in the PLS-SEM setting. Overall, this paper provides researchers and practitioners with the required knowledge to properly carry out, report, and interpret CoMe analysis in PLS-SEM.
\end{abstract}

Keywords: PLS-SEM; Partial Least Squares; Structural Equation Modeling; Conditional Mediation Analysis; Conditional Mediation Model; Conditional Indirect Effect.

\section{Introduction}

Partial least squares structural equation modeling (PLS-SEM) has remained prevalent in information systems, business, and social science research (Hwang et al., 2020; Khan et al., 2019; Petter, 2018). Current advances in PLS-SEM enable a variety of nuanced analyses and encourage use of further robustness checks (Sarstedt et al., 2020b) and predictive assessments (Chin et al., 2020; Hair, 2020; Shmueli et al., 2019; Sharma et al. 2019, 2021) to better interpret PLS-SEM analysis. However, although mediation analysis (Nitzl et al., 2016; Sarstedt et al., 2020a) and moderation analysis (Becker et al., 2018) in PLS-SEM are frequently employed and well understood, the same does not apply to conditional mediation (CoMe) analysis that draws on both. Indeed, extant PLS-SEM applications of CoMe analysisoften referred to differently-vary in their execution and how they are interpreted. Hence, this paper seeks to provide guidance to proficiently conduct CoMe analysis in PLS-SEM.

Applications of CoMe analysis in a PLS-SEM context are scarce. Based on a search of papers published from 2000 to 2019 , we could identify only 27 papers that in some way considered CoMe in PLS-SEM. Eleven of them appeared to disregard the use of a continuous variable when conducting CoMe analysis in PLS-SEM (see, e.g., Lee-Rodríguez et al., 2014; Ahadzadehet et al., 2018; Koay, 2018; Park et al., 2019). Instead, they employed a separate PROCESS 
macro in their analysis. Meanwhile, 16 studies performed multigroup analysis (MGA) when conducting CoMe analysis using a categorical variable. However, the majority of these studies (14 studies) did not examine the difference in the mediated effect before comparing each group's mediated effect values (see, e.g., Calvo-Mora et al., 2016; Fu et al., 2018; Willems et al., 2019; Limaj \& Bernroider, 2019), except for two studies from Rezaei and Valaei (2017) and Hernandez-Ortega et al. (2017). Furthermore, many prior studies have remained vague in specifying whether the theoretical reasoning for their hypothesized CoMe relationship(s) concerns the rate of change (slope) or difference in strength (magnitude) of the effect(s). Clearly, the use of CoMe analysis in PLS-SEM is still in its infancy, which is of fundamental concern to researchers seeking to employ PLS-SEM when assessing theoretical arguments and deriving robust managerial recommendations that rest on relationships that are mediated but conditional on certain exogenous variables.

CoMe analysis can concern distinct types of CoMe models (see Hayes, 2018), which in turn require different analyses. However, some researchers appear to be not familiar with these requirements when conducting PLS-SEM analysis. Furthermore, many researchers still combine their PLS-SEM analyses with Hayes's (2017) PROCESS macros for SPSS or SAS. This is not surprising, because assessing whether a mediated relationship is moderated is relatively easy using a PROCESS macro. Recently, however, Sarstedt et al. (2020a) provided several reasons why this combination is not advisable. They stressed that simultaneous estimations in a nomological network, in contrast to conducting a supplementary regression analysis, are necessary to produce reliable and valid results, especially in the case of multi-item construct measurements. Notably, the sole use of PLS-SEM for estimating a CoMe model offers the following advantages: (1) it overcomes the limitations of traditional sequential approaches by enabling researchers to analyze complex interrelationships between latent variables simultaneously, (2) while accounting for the measurement error inherent in the multi-item measurements (Edwards \& Lambert, 2007; Hayes \& Scharkow, 2013; Muller et al., 2005). Additionally, in PLS-SEM applications, computing the significance of the index of moderated mediation (the CoMe index) and evaluating it remain scarce (Hair et al., 2022). The CoMe index quantifies the effect that a moderator has on a mediated relationship (Hayes, 2015). If the CoMe index is significantly different from zero, a conditional mediated effect is smaller, larger, or opposite in sign at different levels of the moderator. One reason for the infrequent consideration of the CoMe index could relate to the lack of established and adopted conceptual understanding about the nuances of CoMe analysis (i.e., distinguishing between distinct types of CoMe models), in general, and within the PLS-SEM context, in particular. Related, the issue of scarce and sometimes vague applications of CoMe analysis in PLS-SEM likely rests on lacking access to detailed step-by-step procedures for its conduct in the PLSSEM setting. Summarizing these concerns, Sarstedt et al. (2020a) recently noted that clear guidelines for conducting CoMe analysis must be urgently provided; otherwise, its use in PLS-SEM will be hindered.

Overall, this study bridges this gap by offering researchers a guide for estimating and assessing CoMe models in PLS-SEM. This paper first clarifies CoMe analysis. In doing so, the paper illuminates conceptual fundamentals and develops guidelines for CoMe analysis within the PLS-SEM context. Subsequently, the paper outlines the illustrative use of CoMe analysis in and presents detailed step-by-step procedures for the PLS-SEM setting. Together, these conceptual guidelines and practical step-by-step procedures should enable a more proficient CoMe analysis in PLS-SEM applications. In turn, results from CoMe analysis using PLS-SEM in information systems studies should provide a better basis for drawing accurate conclusions to advance theory and produce practical insights.

\section{What is Conditional Mediation Analysis?}

A mediated relationship, also referred to as an indirect relationship, is defined by the presence of one or more variables that intervene to transmit the influence of, for example, variable $X$ on variable $Y$ (Baron \& Kenny, 1986; Pirlott \& MacKinnon, 2016; Zhao et al., 2010). In particular, in mediation analysis, researchers examine whether a change in an independent construct changes a mediator, which in turn results in changes in the dependent construct in the model (Demming et al., 2017). This analysis considers three relationships: a direct path between the independent and dependent variables, a first-stage path (representing the link between an independent variable and the mediator), and a second-stage path (signifying the relationship between the mediator and the dependent variable). In contrast, a moderating effect is defined by the presence of a variable that changes the influence of variable $X$ on variable $Y$ by impacting the nature, direction, or strength of this influence, which can vary under the value of the moderator (Aguinis \& Gottfredson, 2010; Dawson, 2014). Although many guidelines for modeling mediating and moderating effects (see Aguinis et al., 2017; Gardner et al., 2017; Holland et al., 2017; Preacher \& Hayes, 2008) and some directly concerning PLS-SEM (e.g., Becker et al., 2018; Carrión et al., 2017; Nitzl et al., 2016; Sarstedt et al., 2020a) have been widely adopted, explicit 
guidelines concerning the CoMe analysis in PLS-SEM do not exist.

As already indicated, CoMe analysis combines mediation and moderation analyses to examine and test hypotheses about how mediated relationships vary because of context, boundaries, or individual differences. It occurs when a moderator interacts with one or more of the paths of the mediated effect, such that the value of the mediated effect changes depending on the value of the moderator (Hayes, 2017, 2018). This type of modeling is well suited to investigate how relationships between cause and outcomes vary depending on the characteristics of their contexts (Bachl, 2017). For instance, "Does an effect operate through different relationships for certain individuals or under certain circumstances?" or "Is the mediated effect transmitted via some mediators stronger or weaker for some individuals or in some situations than others?" More formally, in a CoMe model, the independent variable of $X$ influences the outcome variable of $Y$ through one or more mediators of $\mathrm{M}$, and the relationship is conditioned by the values of one or more moderators of W and/or Z (Hayes, 2017, 2018).

Scholars in various fields have encouraged researchers to go beyond the separate analysis of mediating and moderating effects as part of the quest to understand and predict theorized effects better (Edwards \& Lambert, 2007; Hayes, 2018; Hayes \& Scharkow, 2013). Two considerations explain why the integration of mediation and moderation can provide a more complete understanding of a phenomenon rather than focusing solely on either mediation or moderation alone as distinct processes (Karazsia et al., 2013). First, examining mediation is not always a universal process. Any bivariate or linear relationship of $X$ on $Y$ can be moderated by a third variable. Thus, researchers may be interested in understanding whether a mediated relationship is moderated by another variable such that the mediated effect value changes depending on the moderator's value (or the mediated effect value is conditional on the moderator's value). Second, the particular process(es) through which a moderating effect occurs may be of considerable interest. Thus, researchers may wish to examine whether a moderator conditions the firststage path or the second-stage path in the mediated relationship, or both. The literature has referred to this type of CoMe analysis also as conditional indirect effect analysis (Edwards \& Lambert, 2007; Hayes, 2015; Preacher et al., 2007).

A CoMe effect represents an effect which is mediated through $\mathrm{M}$, through which $\mathrm{X}$ exerts its influence on $\mathrm{Y}$, and depends on the value of a moderator $W$ (Hayes, 2017). In other words, a CoMe effect is present when the magnitude, size, or direction of the mediated effect of variable $X$ on variable $Y$ via a mediator $M$ varies according to the value of a moderator $Z$ (Preacher et al., 2007). However, many previous studies that purported to test CoMe effects lacked precision in describing how analyses were conducted or draw on different terminology. Hence, the descriptions concerning CoMe analysis vary greatly in literature, making it difficult to fully comprehend what has been done or ought to be done.

In the earlier literature, Muller et al. (2005) discussed mediated moderation in which a single variable moderates the three paths in a simple mediation model. However, this view has been criticized for some of the same concerns that have been raised about the causal-step approach discussed by Baron and Kenny (1986). Subsequently, Morgan-Lopez and MacKinnon (2006) depicted a formal test for both moderated mediation and mediated moderation that researchers could use to establish that an interaction effect on some outcome is carried through a mediator. In their view, mediated moderation refers to the effect of a mediated interaction on a dependent variable, whereas moderated mediation occurs when the strength of the mediated effect depends on the level of a moderator.

Edwards and Lambert (2007) and Preacher et al. (2007) also referred to mediated moderation, but they went far beyond the single model delineated by Muller et al. (2005). Edwards and Lambert (2007) described eight models that could be established by allowing a single variable to moderate one or more causal paths in a mediation process and how the various direct and mediated effects can be assessed. Subsequently, they considered the term mediated moderation to be a specific case of moderated mediation. Nonetheless, this concept is subject to debate. Meanwhile, Preacher et al. (2007) introduced a formal definition of conditional mediation into the lexicon of statistical mediation analysis. They demonstrated how CoMe analysis is conducted to test hypotheses via the construction of standard errors and bootstrap confidence intervals (Cls). They also illustrated how moderated mediation could be conceptualized in a model with two moderators of different paths in the causal system.

Similarly, Hayes and Preacher (2013) and Hayes (2017) challenged the relevance of the very notion of mediated moderation by Edwards and Lambert (2007) and advised against using this term because the corresponding analysis provides no additional insights into the path model effects. Specifically, they argued that the interaction term in a mediated moderation model "has no substantive grounding in the measurement or manipulation process. The product does not quantify anything. And if ... [the interaction term] has no meaning and no substantive 
interpretation, then what does the indirect effect of a product mean? The answer, in my opinion, is that it means nothing" (Hayes, 2017, p. 466). Furthermore, establishing theoretically reasonable underpinnings of mediated moderation models that can be empirically tested is often challenging. For these reasons, Hayes (2017) called to disregard the concept of mediated moderation. Instead, Hayes and Preacher (2013) and Hayes (2017) emphasize moderated (conditional) mediation. In their work, Hayes and Preacher (2013) took a covariance-based structural equation modeling approach in estimating conditional direct and mediated effects simultaneously to demonstrate the evidence of a CoMe effect. Since then, various methodological studies regarding CoMe analysis have appeared to address the means of conceptualizing and quantifying the contingencies of a mediated effect (Borau et al., 2015; Wang \& Preacher, 2015).

In this paper, we use to the term "CoMe model" when referring to the theoretical (research) model. The term "CoMe analysis" denotes the statistical assessment carried out when analyzing and estimating a CoMe model. And the term "CoMe effect" represents the value that is generated through CoMe analysis and quantifies the mediated effect conditional on a moderator.

\section{Conceptual Fundamentals of and Guidelines for Conducting Conditional Mediation Analysis in PLS-SEM}

To conduct CoMe analysis in PLS-SEM, this paper proposes a conceptual logic reflected in a decision tree (Figure 1). It captures conceptual fundamentals and encapsulates guidelines for CoMe analysis in PLS-SEM. Distinguishing between two goals that may guide the CoMe analysis, we outline two general paths of actions that ought to be followed.

\section{The Analysis Goal}

Commonly, researchers would be theory-driven, not data-driven, in their research design, especially when testing a hypothesized research model. This means that researchers would normally develop appropriate hypotheses that they seek to test drawing, in our case, on a CoMe model. Hair et al. (2022) stated that hypotheses are individual conjectures and are developed following the scientific method used to explain and predict outcomes. It allows researchers to follow a set of formal steps in conducting calculated analysis on their data. Theory-driven hypotheses can take on either of the following two forms when specifying a CoMe model, which concern either the rate of change (or slope) or difference in the strength (or magnitude) of a relationship:

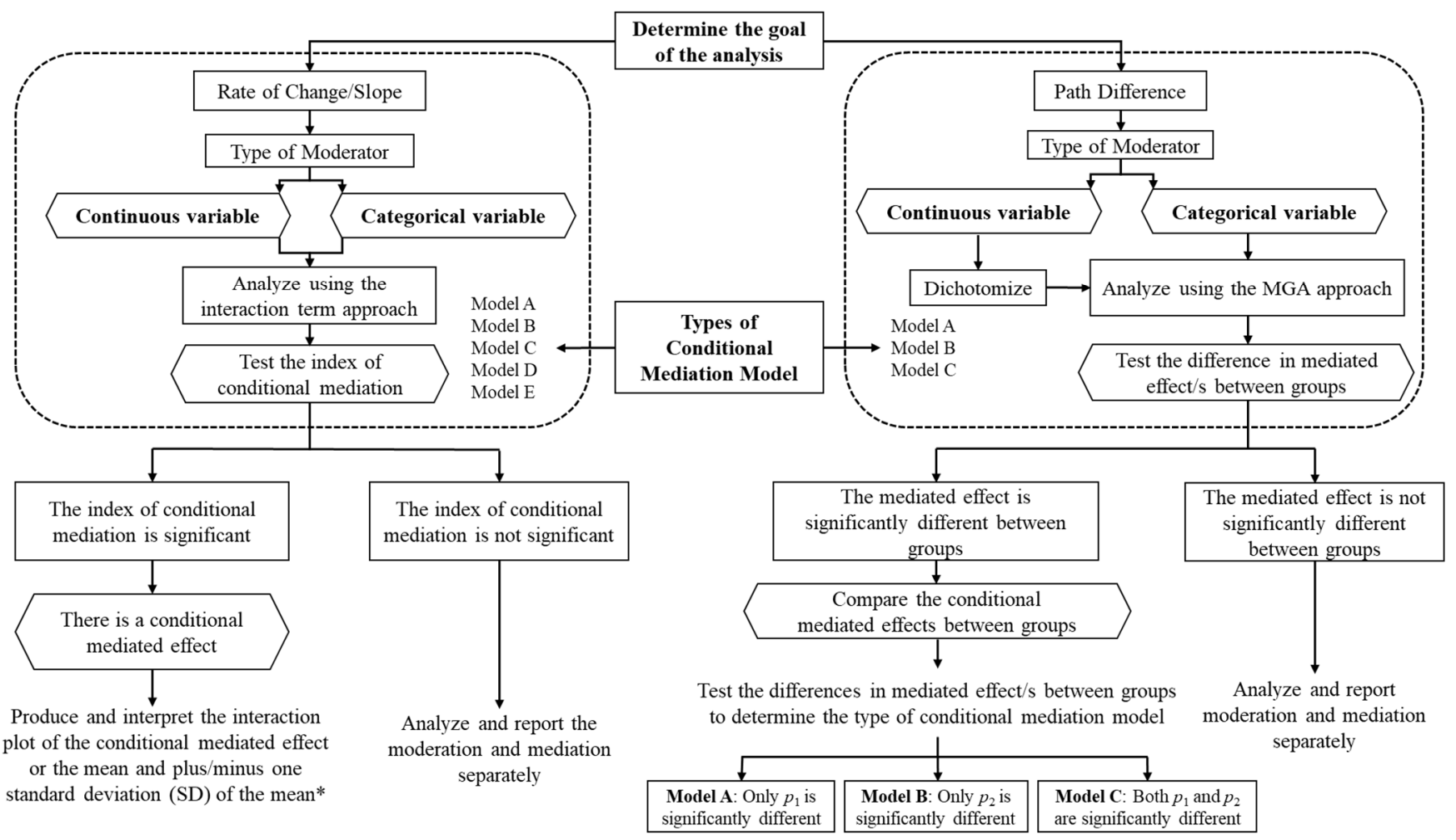

Note: * indicates that both \pm 2 SD or \pm 3 SD can be easily adopted if a researcher is interested in obtaining wider intervals of SD.

Figure 1. Decision Tree of Conditional Mediation Analysis in PLS-SEM 
1. The mediated relationship (M) between $X$ and $Y$ increases as $\mathrm{W}$ increases (vice-versa).

2. The mediated relationship (M) between $X$ and $Y$ is greater for high values of W (or Group A) than for low values of W (or Group B) (vice-versa).

Drawing from the above mentioned, any research design to test a CoMe model must be informed by underlying theory. Underlying theoretical reasoning has two crucial implications. First, it substantiates the distinct type of the CoMe model considered (please see latter sections for more details on distinct types of CoMe models). Second, the theoretical argument determines whether to assess either the rate of a slope's change or differences in the strength (i.e., magnitude) of a path. To answer this question, if researchers seek to assess the first type of hypothesis (i.e., rate of change/slope), they should assess and interpret the interaction term, whereas if researchers seek to assess the second type of hypothesis (i.e., differences in the strength/magnitude of a path), then they must compare the path values of two or more different groups. This means that the theoretical argument ought to drive the goal of the analysis; in turn, the choice of variable-regardless of continuous or categorical moderator-and the approach of estimation (interaction term or MGA), which are explained in the latter parts of this paper. Importantly, when the analysis goal is pre-determined via a hypothesis, it should enable the researcher to specify and implement a suitable research design that, in turn, guides the collection of appropriate data to fit the required estimation approach to assess the hypothesis.

\section{Type of Moderating Variable}

Two types of moderating variables can be embedded in CoMe analysis: continuous and categorical moderating variables. When a moderator is continuous (e.g., switching costs, firm resources, or privacy concerns), researchers can generate an interaction term that expresses the joint influence of the exogenous construct and moderator on the endogenous construct (see Hair et al., 2022). This approach would suit estimating the rate of change (i.e., slope) of a relationship. Alternatively, when a moderator is categorical (e.g., industry, gender, or nationality), the variable may serve as a grouping variable that divides the data into subsamples. The theoretical model is then estimated for each distinct subsample. Researchers are usually interested in understanding any significant differences between subsamples; thus, the model estimates for the subsamples are usually compared using MGA (Sarstedt et al., 2011). The latter approach would enable assessments of differences in the strength (i.e., magnitude) of one or more paths.

\section{Conditional Mediation Analysis with Continuous Moderators}

An index term analysis should be conducted when the moderator is continuous and the goal of the CoMe analysis is to assess the rate of change (or slope) of a relationship. An "index term is known as the index of moderated mediation" that can be defined as a "direct quantification of the linear association between the indirect effect and the putative moderator of that effect" (Hayes, 2015, p. 3). As indicated in an earlier section of this paper, we refer to this index of moderated mediation as the CoMe index $(\omega)$. Researchers should be clear about the relationships on which the moderator exerts its influence when estimating this index term. In this vein, Figures 2-6 illustrate the five types of CoMe models (Models A-E) that allow a mediated relationship to be moderated by a continuous type of moderator. The four most popular forms of CoMe models are Model A (the first-stage conditional process model), Model $B$ (the secondstage conditional process model), and both Models $C$ and $\mathrm{D}$ (the first- and second-stage conditional process models); Model E is less common. ${ }^{1}$ Notably, this present study does not illustrate the moderating effect on the mediated relationship between $X$ and $Y$ (also known as conditional direct effect) ${ }^{2}$ because even though a moderating effect may exist on the direct relationship of the CoMe model, the focus of the statistical estimation is only based on $\mathrm{X}$ and $\mathrm{M}$ on $\mathrm{Y}$.

In CoMe Model A (see Hayes, 2017, 2018), moderator $W$ is assumed to affect the first stage of the mediation process (Figure 2). W moderates the effect of $X$ on $M$, but the effect of $M$ on $Y$ is independent of $W$. To substantiate that the mediation is moderated, researchers traditionally note the need for evidence of a significant moderation of the path linking $X$ and $M$ (Muller et al., 2005; Preacher et al., 2007), which is equivalent to testing the significance of path $\left(p_{5}\right)$ in Figure 2. However, Hayes and Rockwood (2020) have emphasized that a nonsignificant moderation result does not necessarily imply that $W$ does not moderate the mediated effect of $X$ on $Y$. Additionally, researchers must consider the impact of the moderator on the mediated effect as a whole rather than on a single element of the mediated effect in isolation (in this case, path $p_{2}$ ) (Hayes, 2018). To formally test this effect in CoMe Model A, Hayes (2018) proposed the CoMe effect, which is defined as follows:

$$
\omega=p_{1} \cdot p_{2}+p_{2} \cdot p_{5} \cdot W_{(1)}
$$

Based on equation 1 , the path coefficients of " $p_{2} \cdot p_{5}$ " refer to the interrelationships of $M$ between the constructs of $X$ and $Y$ (Figure 2), whereas $W$ refers to the moderator. If the index " $p_{2} \cdot p_{5}$ " is significantly different from zero, the mediated effect of $X$ on $Y$ through $\mathrm{M}$ depends on moderator $\mathrm{W}$. In other words, 
" $p_{2} \cdot p_{5}$ " is the slope of the mediated effect of $\mathrm{W}$ contingent upon $\omega$, which represents the CoMe index (Hayes, 2015; Morgan-Lopez \& MacKinnon, 2006). The index is a product of path coefficients; hence, a bootstrap-based percentile confidence interval should be used for inference without any assumption about the shape of its sampling distribution. ${ }^{3}$ The CoMe index $(\omega)$ can be easily obtained based on the path coefficient output generated by any software that enables running of PLS-SEM algorithms (e.g., R, SmartPLS, WarpPLS, ADANCO, etc.).

\section{Conceptual Model}

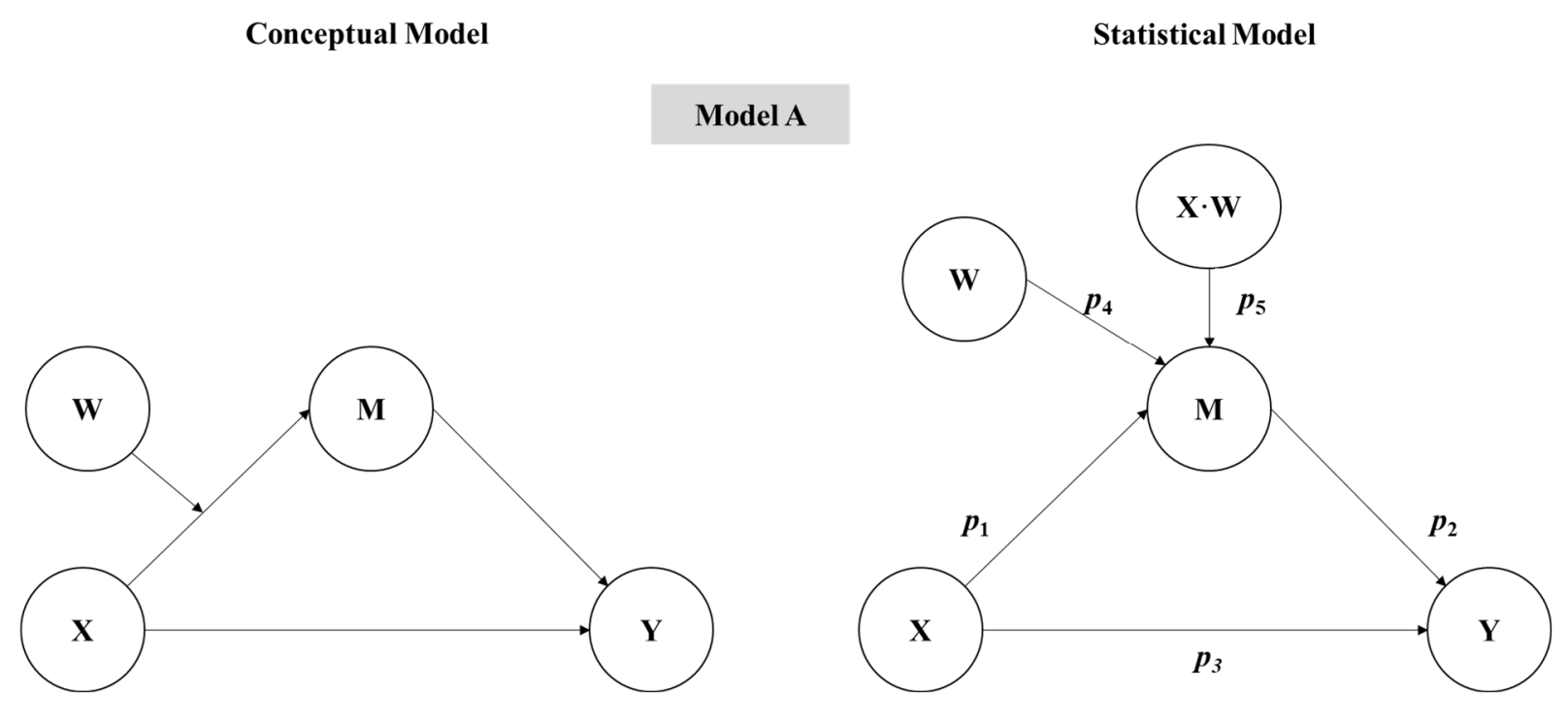

The second way to combine mediation and moderation is through the second-stage conditional process model (Figure 3). In CoMe Model B, moderator $\mathrm{W}$ affects only the second stage of the mediation process, in which the relationship between the mediator $M$ and endogenous variable $Y$ is assumed to be moderated by W. In other words, this second-stage conditional process model fixes the effect of $X$ on $M$ to be constant but allows the effect of $\mathrm{M}$ on $\mathrm{Y}$ to be moderated by $\mathrm{W}$. To assess the occurrence of this second-stage conditional process model, Hayes (2018) defined the CoMe effect as follows:

Figure 2. CoMe Model A

Conceptual Model

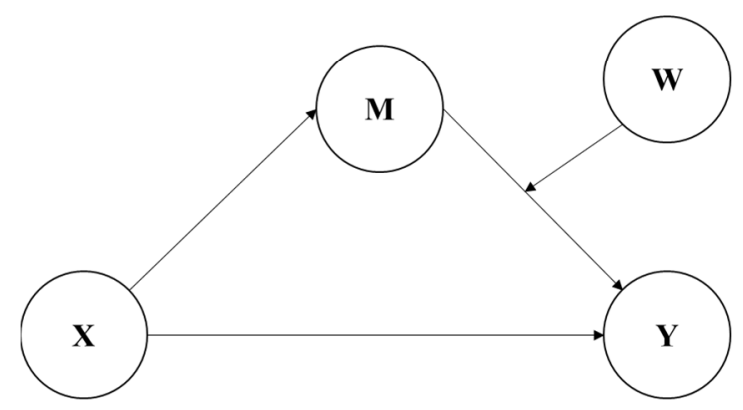

Statistical Model

Model B

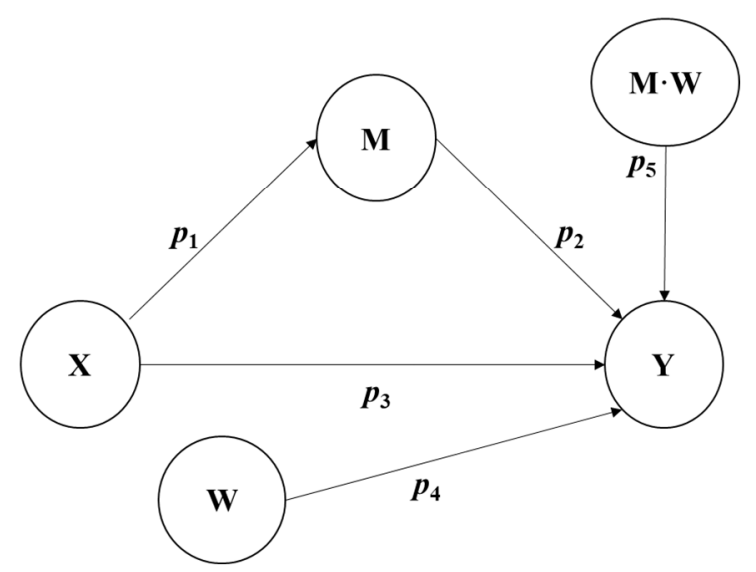

Figure 3. CoMe Model B 


$$
\omega=p_{1} \cdot p_{2}+p_{1} \cdot p_{5} \cdot W_{(2)}
$$

Based on equation 2, if the index " $p_{1} \cdot p_{5}$ " is significant, the mediated effect through $M$ is significantly moderated by $W$. The index also quantifies the effect of $W$ on the mediated effect of $X$ on $Y$ through $M$. However, at present, such an output of the CoMe index $(\omega)$ cannot yet be directly obtained from any type of PLS-SEM software (R, SmartPLS, WarpPLS, ADANCO, etc.). Hence, the path coefficient results must be examined using the bootstrap sample output. For example, a researcher can copy the bootstrap results into an Excel spreadsheet and multiply all lines (e.g., 5,000 or 10,000 subsample bootstraps) of $p_{1}$ and $\mathrm{p}_{5}$, to calculate the bootstrap Cls from the data in this new calculated column. Alternatively, using other software packages such as the $R$ software is an efficient way for generating this result (i.e., $p_{1} \cdot p_{5}$ and the bootstrap Cls) (R Core Team, 2021). The third type of CoMe model, shown in CoMe Model C, is the combination of the first- and second-stage conditional process models, in which $W$ simultaneously moderates the effects of both $X$ on $M$ and $M$ on $Y$ (Figure 4). The proposed conditional effect is specified like any mediation model $\left(p_{1} \cdot p_{2}\right)$ but includes the product for testing the moderating effect of both $\mathrm{X} \cdot \mathrm{W}$ on $M$ and $M \cdot W$ on $Y$. Hayes (2018) defined the following conditional effect equation for CoMe Model C:

$$
\omega=p_{1} \cdot p_{2}+\left(p_{2} \cdot p_{5}+p_{1} \cdot p_{7}\right) \mathrm{W}+p_{5} \cdot p_{7} \mathrm{~W}^{2}(3)
$$

In this equation, " $p_{2} \cdot p_{5}+p_{1} \cdot p_{7}$ " is the CoMe index $(\omega)$ for CoMe Model $C$. When this sum of the products is significant, the CoMe effect occurs; that is, the mediated effect of $X$ on $Y$ through $M$ is moderated by W.
The fourth type of CoMe model, shown in CoMe Model $D$ (Figure 5), is similar to CoMe Model C. The only difference is that CoMe Model $D$ has two distinct moderators influencing the mediated effect. Particularly, $W$ moderates the effect of $X$ on $M$, and $Z$ moderates the effect of $M$ on $Y$. Additionally, the proposed conditional effect is specified like any mediation model $\left(p_{1} \cdot p_{2}\right)$ but includes both the product of $X \cdot W$ and $M \cdot Z$ in the models of the effect of $X$ on $Y$ through M (Hayes, 2018). This type of CoMe effect equation is defined as follows:

$$
\omega=p_{1} \cdot p_{2}+\left(p_{2} \cdot p_{5}\right) \mathrm{W}+\left(p_{1} \cdot p_{7}\right) \mathrm{Z}+p_{5} \cdot p_{7} \mathrm{WZ}(4)
$$

In this equation, " $p_{2} \cdot p_{5}+p_{1} \cdot p_{7}$ " is the CoMe index $(\omega)$ for CoMe Model $D$. When this sum of products is significant, the CoMe effect occurs; that is, the mediated effect of $X$ on $Y$ through $M$ is moderated by $W$ and $Z$ respectively.

Finally, Figure 6 presents the fifth type of the CoMe model. In CoMe Model E, X moderates its own mediating effect on $\mathrm{Y}$ through $\mathrm{M}$ via the moderation of the effect of $M$ on $Y$ by $X$. Here, the independent variable is the moderator model, and the mediated effect of $X$ on $Y$ through $M$ is the product of the effect of $X$ on M. Hayes (2017) defined the following conditional effect equation for CoMe Model E:

$$
\omega=p_{1} \cdot p_{2}+p_{1} \cdot p_{4} \cdot X_{(5)}
$$

In this equation, " $p_{1} \cdot p_{4}$ " is the CoMe index $(\omega)$ for CoMe Model E (Hayes, 2015). It quantifies the relationship between $X$ and the mediated effect of $X$ on $Y$ through $M$. $X$ functions as a moderator of its own mediated effect.

\section{Conceptual Model}

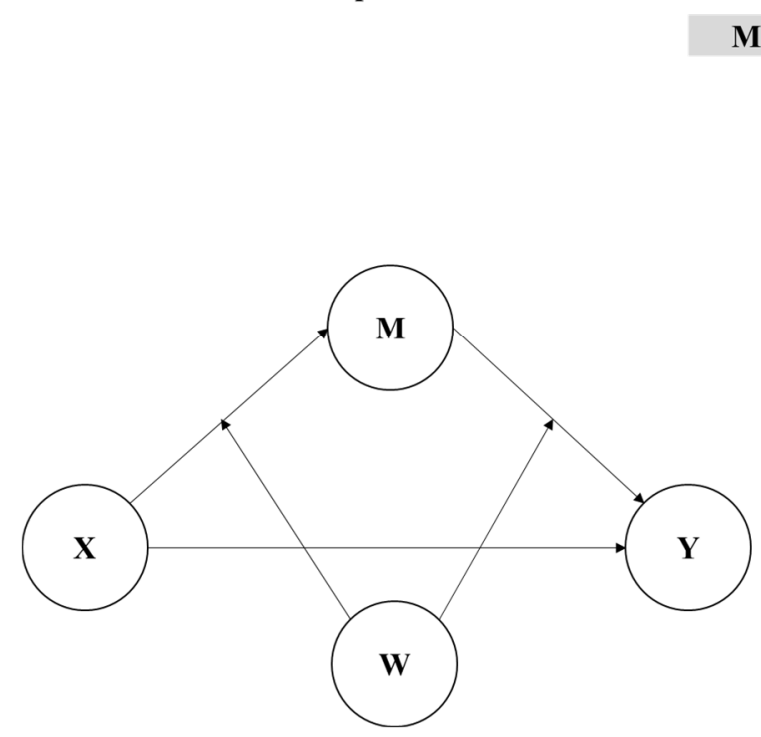

Statistical Model

Model C

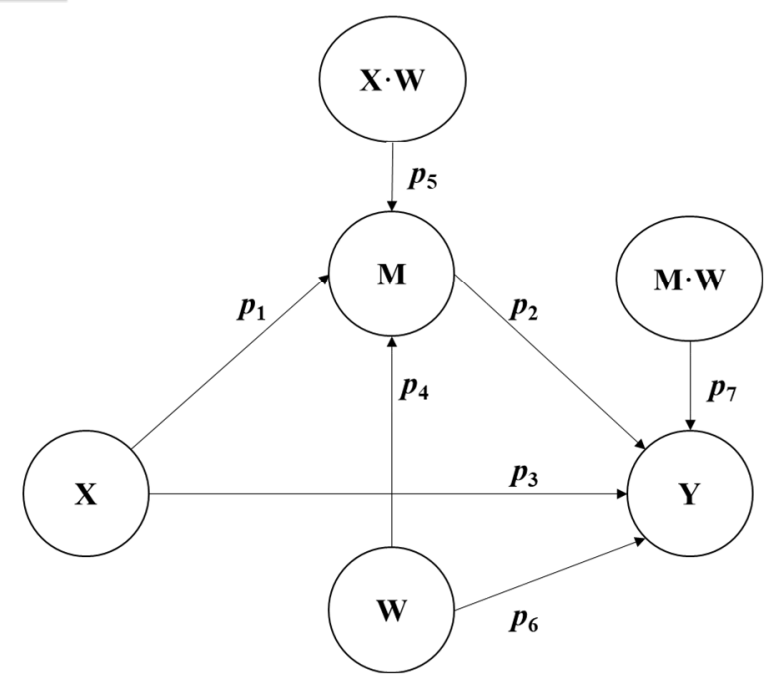

Figure 4. CoMe Model C 


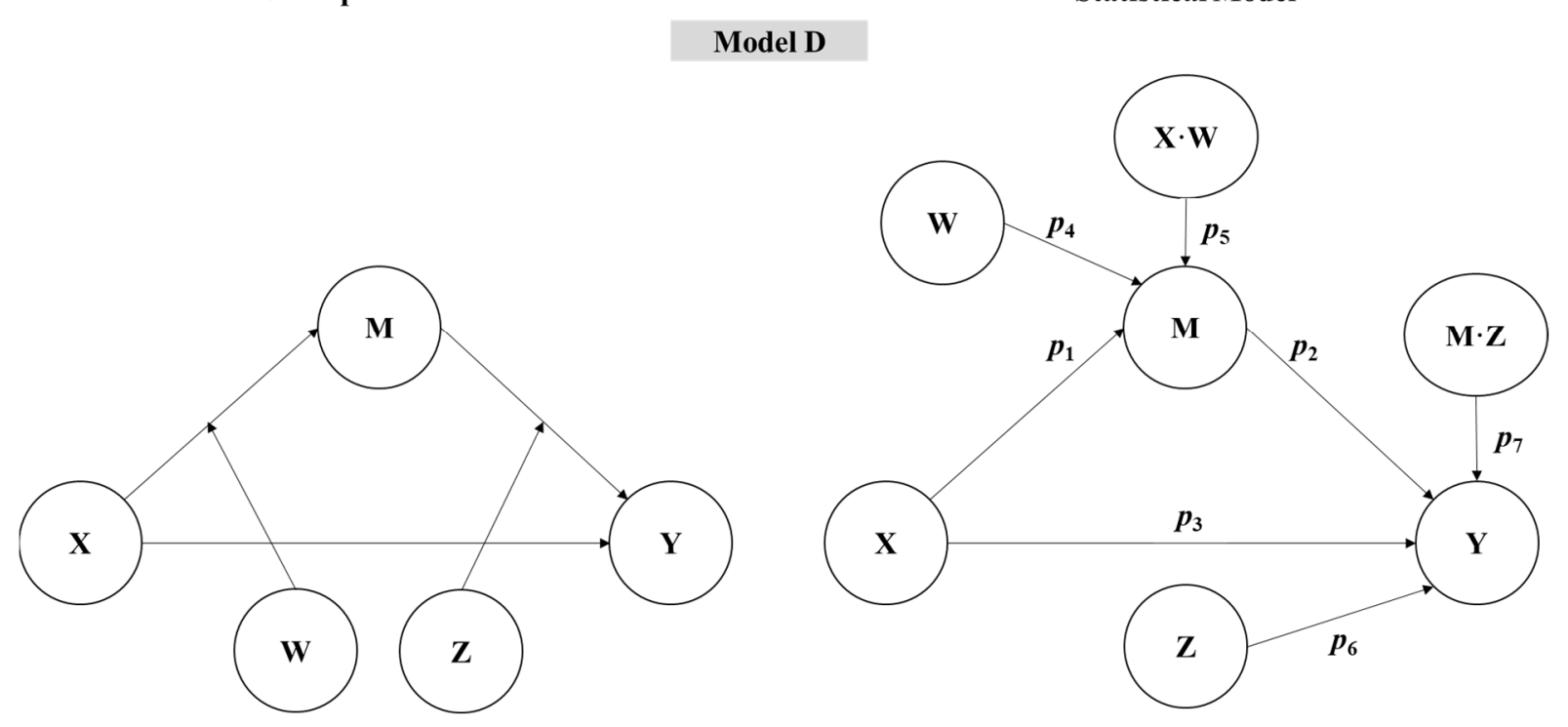

Figure 5. CoMe Model D

Conceptual Model

Statistical Model

Model E
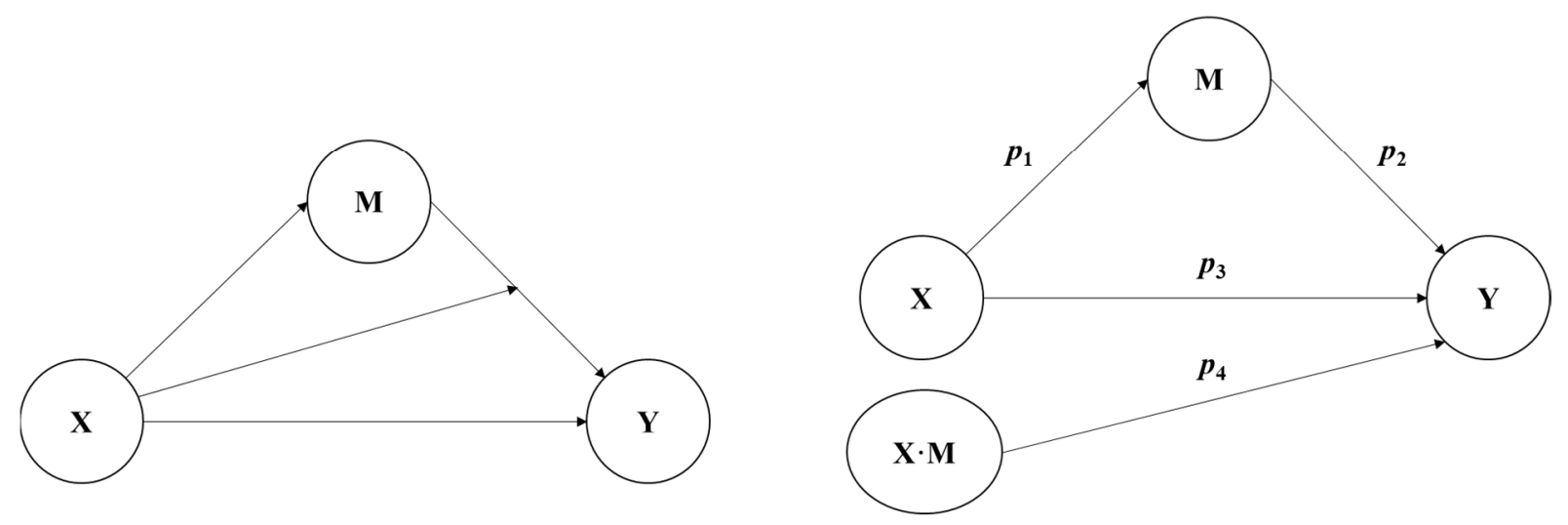

Figure 6. CoMe Model E

\section{Conditional Mediation Analysis with (Multi)Categorical Moderators}

When $\mathrm{W}$ is a categorical (or dichotomous) moderator (possibly transformed from a continuous variable ${ }^{4,5}$ ), we can conduct an interaction analysis for a CoMe model if the research goal is to study the rate of change (or slope) of a relationship. These variables are usually dummy coded (i.e., 0 and 1 ), whereby 0 represents the reference category (Henseler \& Fassott, 2010; Wooldridge, 2015). For instance, suppose $W$ is gender, then the researcher could use a variable with 0 for "Male" and 1 for "Female" (vice-versa). Meanwhile, when $\mathrm{W}$ is a dichotomous variable represented in the data with values that differ by one unit (e.g., $W=0$ and $W=1$ ), the CoMe index $(\omega)$ is the difference between the mediated effect of $X$ on $Y$ in the two groups. In referring to Equation 1 (the same applies similarly for equations $2-5$ ), when $\mathrm{W}$ acts as a categorical moderator in the CoMe model, the indexes of " $p_{2} \cdot p_{5}$ " will be tested as usual; that is, the two values of $W$ chosen to code the two groups can be used in place of $\omega$.

However, in many studies, $\mathrm{W}$ is multi-categorical, for example, (1) a control group versus two or more experimental conditions and (2) the positions on $\mathrm{W}$ could be three values on a continuous variable that are 
operationalized "high," "medium," and "low" on that variable. In such a scenario, the researcher should rely on the fact that CoMe effect differences can be estimated with a linear model by representing groups with a set of $k-1$ variable, where $k$ is the number of groups that should be transformed into as many dummy variables as there are levels (Henseler \& Fassott, 2010; Wooldridge, 2015). For example, in the case of three groups suggested by Hair et al. (2022) (e.g., for a service-type variable: short-term contract, long-term contract, and prepaid), researchers can split the moderator into two dummy variables, which are simultaneously included in the model. Particularly, both dummy variables would take the value of 0 for the reference category (e.g., prepaid). The other two categories would be indicated by the value 1 in the corresponding dummy variable: dummy 1 (prepaid: 0 vs. short-term contract: 1 ) and dummy 2 (prepaid: 0 vs. long-term contract: 1).

\section{Generating and Testing Interaction Terms in Conditional Mediation Analysis}

To generate the interaction term, Becker et al. (2018) and Fassott et al. (2016) highlighted three potential procedures, namely, the product-indicator approach (Chin et al., 2003), the orthogonalizing approach (Lance, 1988), and the two-stage approach (Henseler \& Fassott, 2010). The latter approach is more commonly considered as the more suitable one (see Hair et al., 2022). When interpreting the results of a moderation analysis, scholars are primarily interested in the significance of the interaction term. Suppose that the effect of the interaction term significantly moderates at least one path in the causal process linking $X$ to $Y$ via $M$. When the remaining unmoderated path is also significantly different from zero, the presence of a CoMe effect could exist (Muller et al., 2005). However, PLS-SEM researchers should conduct a formal test to assess the CoMe index $(\omega)$ because a mediated effect can be moderated even if one cannot show significant moderation of its causal process linking $X$ to $Y$ via $M$.

Importantly, before estimating these several types of CoMe models (Models A-E), approaches that gauge measurement error must be considered (Hair \& Sarstedt, 2019). Doing so is important to monitor the quality of the variables' measurement models, in consideration of either reflective (Mode A estimation) or formative (Mode $B$ estimation) measurement modes. ${ }^{6}$ Particularly, if a reflective measurement mode is used, researchers must ensure that the indicator loading has a value of at least 0.708 with an associated significant result (Hair et al., 2022). Then, the measurements must be considered reliable (i.e., indicator reliability, Cronbach's alpha, and composite reliability) and valid (i.e., convergent validity and discriminant validity) to detect a CoMe effect (see Hair et al., 2020). Alternatively, if we have a formative measurement mode, convergent validity (Cheah et al. 2018), variance inflation factor (Becker et al., 2015; Mason \& Perreault Jr, 1991), and the statistical significance and relevance of the indicator weights must be monitored (Hair et al., 2022; Hair et al., 2019).

Once the properties of the measurement models for the constructs embedded in the CoMe model are validated (irrespective of whether reflective or formative), the researcher can then start to examine the CoMe index $(\omega)$ using the bootstrapping technique. Particularly, to establish the presence of the CoMe index $(\omega)$, the researcher must observe 90 percent or 95 percent $\mathrm{Cl}$ of index $\omega .^{7}$ If the CoMe index $(\omega)$ is significantly different from zero (or statistically significant), researchers can conclude that the mediated effect of $X$ on $Y$ through $M$ depends on $W$. This allows establishing a CoMe effect. Vice-versa, if the CoMe index $(\omega)$ is not statistically significant, the CoMe effect is not verified. Even though a mediated effect may exist, it does not depend on the level of the moderator. The lack of significance of the CoMe effects for low, medium, and high levels of the moderator means that the moderator cannot produce a differential effect. A higher level of the moderator leads to a similar effect as the lower level of the moderator. Therefore, PLS-SEM researchers should subsequently analyze the mediation and moderation separately because the results might be individually significant in explaining a mediated or interaction effect.

\section{Conditional Mediation Analysis at Different Levels of the Moderator}

Once the existence of a CoMe effect is supported by the CoMe index $(\omega)$, one may wish to investigate the mediated effect at representative levels (or values) of the moderator (depicted as the CoMe effect) to explore further the conditions under which mediation exists (Preacher et al., 2007). Specifically, researchers must determine how the moderator at different levels (or values) influences the mediated effect, which may be stronger, weaker, or opposite in sign. For a continuous moderator, by default, the CoMe effects are tested based on the mean of the moderator and at values of one or two standard deviations (SDs) below (or low), average (or medium), and above (or high) the mean using the following equations in Table 1. 
Table 1. CoMe Effect Testing at Different Levels of the Moderator

\begin{tabular}{|c|c|}
\hline Model & Equation \\
\hline$A$ & $\begin{array}{ll}\text { - } & \text { CoMe effect }(\text { low })=\left[\left(p_{1}+\left(p_{5} \cdot-\mathrm{SD} \text { of } \mathrm{W}\right)\right) \cdot p_{2}\right](6) \\
\text { - } & \text { CoMe effect }(\text { medium })=\left[\left(p_{1}+\left(p_{5} \cdot \mathrm{SD} \text { of } 0 \text { for } \mathrm{W}\right)\right) \cdot p_{2}\right](7) \\
\text { - } & \text { CoMe effect }(\text { high })=\left[\left(p_{1}+\left(p_{5} \cdot+\mathrm{SD} \text { of } \mathrm{W}\right)\right) \cdot p_{2}\right](8)\end{array}$ \\
\hline$B$ & $\begin{array}{ll}\text { - } & \left.\text { CoMe effect }(\text { low })=\left[p_{1} \cdot\left(p_{5} \cdot-\mathrm{SD} \text { of } \mathrm{W}\right)+p_{2}\right)\right]_{(9)} \\
\text { - } & \left.\text { CoMe effect (medium })=\left[p_{1} \cdot\left(p_{5} \cdot \mathrm{SD} \text { of } 0 \text { for } \mathrm{W}\right)+p_{2}\right)\right](10) \\
\text { - } & \left.\text { CoMe effect }(\text { high })=\left[p_{1} \cdot\left(p_{5} \cdot+\mathrm{SD} \text { of } \mathrm{W}\right)+p_{2}\right)\right]_{(11)}\end{array}$ \\
\hline $\mathrm{C}$ & $\begin{array}{ll}\text { - } & \text { CoMe effect }(\text { low })=\left[\left(p_{1}+\left(p_{5} \cdot-\mathrm{SD} \text { of } \mathrm{W}\right)\right) \cdot\left(\left(p_{7} \cdot-\mathrm{SD} \text { of } \mathrm{W}\right)+p_{2}\right)\right](12) \\
\text { - } & \text { CoMe effect }(\text { medium })=\left[\left(p_{1}+\left(p_{5} \cdot \mathrm{SD} \text { of } 0 \text { for } \mathrm{W}\right)\right) \cdot\left(\left(p_{7} \cdot \mathrm{SD} \text { of } 0 \text { for } \mathrm{W}\right)+p_{2}\right)\right](13) \\
\text { - } & \text { CoMe effect }(\text { high })=\left[\left(p_{1}+\left(p_{5} \cdot+\mathrm{SD} \text { of } \mathrm{W}\right)\right) \cdot\left(\left(p_{7} \cdot+\mathrm{SD} \text { of } \mathrm{W}\right)+p_{2}\right)\right]_{(14)}\end{array}$ \\
\hline $\mathrm{D}$ & $\begin{array}{ll}\text { - } & \text { CoMe effect }(\text { low })=\left[\left(p_{1}+\left(p_{5} \cdot-\mathrm{SD} \text { of } \mathrm{W}\right)\right) \cdot\left(\left(p_{7} \cdot-\mathrm{SD} \text { of } \mathrm{Z}\right)+p_{2}\right)\right]_{(15)} \\
\text { - } & \text { CoMe effect }(\text { medium })=\left[\left(p_{1}+\left(p_{5} \cdot \mathrm{SD} \text { of } 0 \text { for } \mathrm{W}\right)\right) \cdot\left(\left(p_{7} \cdot \mathrm{SD} \text { of } 0 \text { for } \mathrm{Z}\right)+p_{2}\right)\right]_{(16)} \\
\text { - } & \text { CoMe effect }(\text { high })=\left[\left(p_{1}+\left(p_{5} \cdot+\mathrm{SD} \text { of } \mathrm{W}\right)\right) \cdot\left(\left(p_{7} \cdot+\mathrm{SD} \text { of } \mathrm{Z}\right)+p_{2}\right)\right]_{(17)}\end{array}$ \\
\hline$E$ & $\begin{array}{ll}\text { - } & \left.\text { CoMe effect }(\text { low })=\left[p_{1} \cdot\left(p_{4} \cdot-\mathrm{SD} \text { of } \mathrm{X}\right)+p_{2}\right)\right]_{(18)} \\
\text { - } & \left.\text { CoMe effect }(\text { medium })=\left[p_{1} \cdot\left(p_{4} \cdot \mathrm{SD} \text { of } 0 \text { for } \mathrm{X}\right)+p_{2}\right)\right]_{(19)} \\
\text { - } & \left.\text { CoMe effect (high) }=\left[p_{1} \cdot\left(p_{4} \cdot+\mathrm{SD} \text { of } \mathrm{X}\right)+p_{2}\right)\right]_{(20)}\end{array}$ \\
\hline
\end{tabular}

By simplifying the algebraic expressions, we can derive the equations in Table 2 from equations $1-5$ (see Hayes, 2017; Stride et al., 2015). To clarify these equations, we draw on explaining this for CoMe Model A. ${ }^{8}$ In the initial step, the researcher must run bootstrapping for each path coefficient to obtain the bootstrap subsample result (i.e., 5,000 or 10,000 subsamples) (Hair et al., 2022; Streukens \& LeroiWerelds, 2016a). Then, the researcher can take the path coefficient from the interaction term to the mediator $\left(p_{5}\right)$ and multiply it by the SD values of $\mathrm{W}$ (the moderator), which represent low (mean-1 SD of the moderator), medium (mean of the moderator), and high (mean $+1 \mathrm{SD}$ of the moderator) levels. ${ }^{9}$ Next, the researcher must take these three values and multiply them by the path coefficient from the independent variable to the mediator $\left(p_{1}\right)$. Finally, the product of these values will be multiplied by the path coefficient from the mediator to the dependent variable $\left(p_{2}\right)$ to obtain CoMe effects for low, medium, and high levels of the moderator.
Conversely, if $\mathrm{W}$ is a (multi)categorical moderator, the researcher can follow the procedures by taking the path coefficient from the interaction term to the mediator $\left(p_{5}\right)$ and multiplying it by the SD values of $\mathrm{W}$ (similar to Models B-E). The only difference is that the researcher cannot treat it as CoMe effects for low, medium, and high levels of the moderator; rather, the dummy is compared with the reference category.

Finally, the researcher can create a graphical plot as part of visualization the data (Spiller et al., 2013) to corroborate the findings of the CoMe effects, that is, to investigate the significance of each condition (CoMe effects for low, medium, and high levels of the moderator) at bounds of the CoMe index $(\omega)$ (Preacher et al., 2007). Particularly, the researcher can use the simple slope or Johnson-Neyman's plot suggested by Preacher et al. (2007) to apply the regions of significance approach or identify the ranges of the moderator for which a mediated effect is statistically significant. 


\section{Conditional Mediation Analysis Using MGA}

In most cases, where the moderator $(\mathrm{W})$ is categorical and the goal is to understand the differences in the strength (magnitude) or directionality of mediated effects, an MGA can be used to understand the CoMe effect more comprehensively. Notably, the MGA can also be applied drawing on a continuous moderator if the aim is to test the differences in the strength (magnitude) or directionality of mediated effects. In this case, the continuous variable must be dichotomized, however. Once the observations are grouped, models are estimated separately for each group of observations. The differences in the mediated effects between the data groups are interpreted as CoMe effects. Figure 7 depicts the CoMe effect based on comparing effects for two groups, in which the impact of $W$ can be estimated using a nominal variable such as Group A versus Group B or high values versus low values of a particular moderator. Researchers are usually interested in analyzing group effects related to mediated relationships. More precisely, a population parameter $p 1 \cdot p 2$ is hypothesized to be different (d) across two subpopulations (i.e., $p_{1}{ }^{(1)} \cdot p_{2}{ }^{(1)}$ and $\left.p_{1}{ }^{(2)} \cdot p_{2}{ }^{(2)}\right)$, which are expressed by different modalities in the categorical moderating variable.

Additionally, an MGA enables the researcher to test for differences between identical models estimated for different groups. According to Shrout and Bolger (2002), a group comparison can be useful for analyzing mediated effects. For instance, a CoMe effect may occur when the mediated effect of one group is significantly stronger than or even different from that of another group. Such groups can, for example, relate to observable characteristics (e.g., gender) or refer to experimental conditions (e.g., high value of $W$ versus low value of $W$ ). For instance, a researcher may assume that $X$ influences $Y$ indirectly through $M$, but the effect is stronger for men (or the high value of $W$ ) than for women (or the low value of W); alternatively, the effect is perhaps present only for men or women (or only for the high or low value of $W$ ) (Hair et al., 2018). Figure 8 shows that full mediation exists in Group A: Male (or the high value of $W$ ), whereas it does not exist in Group B: Female (or the low value of $W$ ).

To test group effects in PLS-SEM, researchers must conduct a measurement invariance test to determine whether construct measurements are similarly suitable across groups or heterogeneous data (Henseler et al., 2016). ${ }^{10}$ In other words, this test concerns the statistical property of an instrument, indicating that the same construct(s) are measured in the same way across subgroups of respondents. The reason for such differences may stem from (1) respondents embracing different cultural values who interpret a given measure in a conceptually different manner or gender, ethnic, or other individual differences that entail responding to instruments in systematically different ways, and (2) respondents who use the available options on a scale differently (or tendency to choose or not choose the extremes) (Hair et al., 2018; Vandenberg \& Lance, 2000).

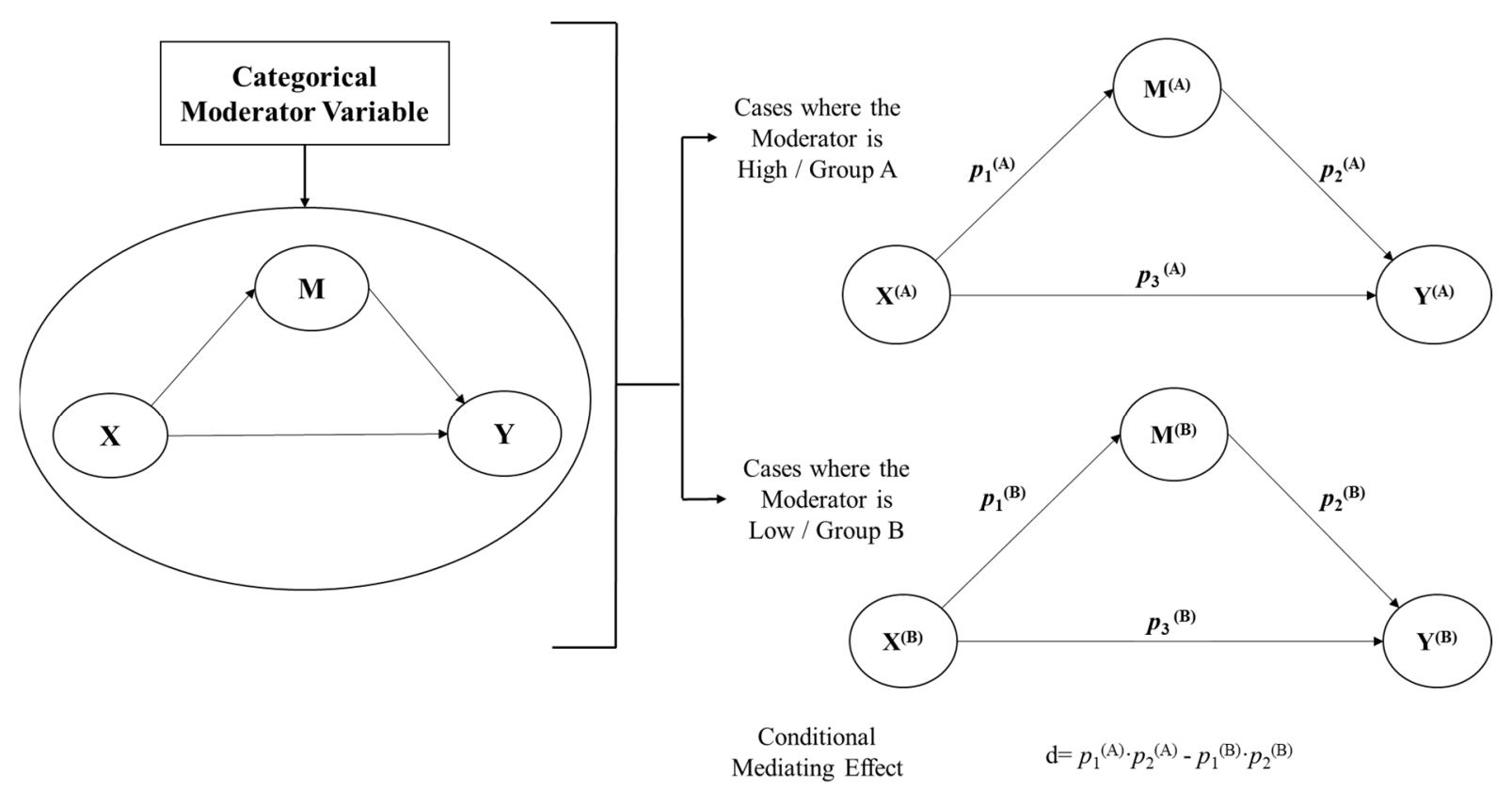

Figure 7. Detecting a Conditional Mediated Effect (d) through Group Comparisons 
Group A: Male (or high value of W)

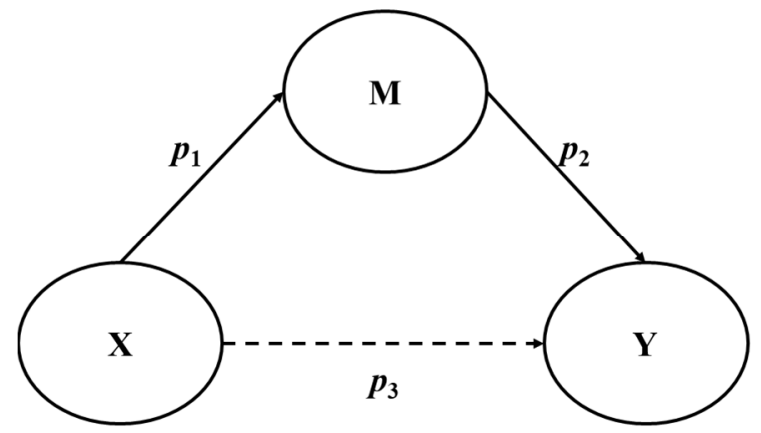

The relationship of $p_{1} \cdot p_{2}$ is significant but $p_{3}$ is insignificant
Group B: Female (or low value of W)

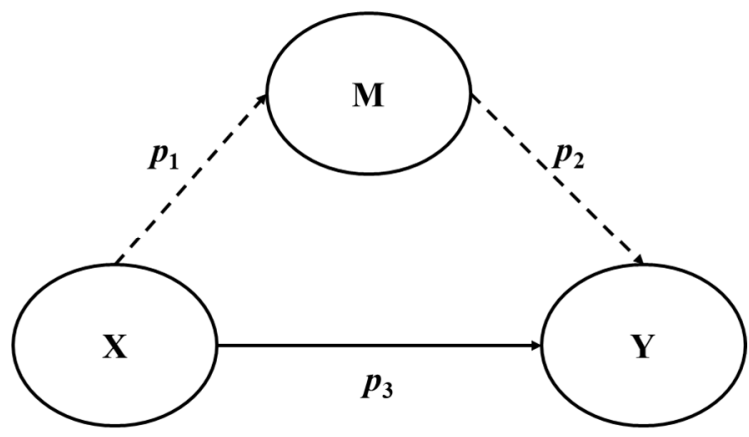

The relationship of $p_{1} \cdot p_{2}$ is nonsignificant but $p_{3}$ is significant

Figure 8. Full Mediation in Testing Group A (or the high value of W) and No Mediation in Testing Group B (or the low value of $W$ )

Once this similarity is established, a group comparison can be estimated via five methods of MGA in PLSSEM: the permutation test (Chin \& Dibbern, 2010), Henseler's bootstrap-based MGA (Henseler et al., 2009), the non-parametric distance-based test (Klesel et al., 2019), the parametric test (Keil et al., 2000), or the Welch-Satterthwaite test (Welch, 1947). Drawing on these available MGA methods allows comparing a model across different groups to determine whether the strength (i.e., magnitude) of one or more relationships in the model is different between groupbased estimations and whether any differences are statistically significant. If the test does not support a significant difference in the mediated effect between groups, the researcher should reject the existence of a CoMe effect. In other words, if the CoMe effect is not significant in the MGA estimation, the PLS-SEM researcher should subsequently analyze mediation and moderation separately.

Before performing an MGA, the researcher should ensure that measurement invariance is achieved drawing on the measurement invariance of composite models (MICOM) procedure (see Henseler et al., 2016). By establishing MICOM, the researcher can be assured that the group differences in the model estimates do not result from distinct content and meanings of measurements used for the latent variables across groups. Notably, conducting an MGA requires partial measurement invariance, which is given when configural and compositional invariance hold. If partial measurement invariance is confirmed and the composites have equal mean values that vary across the groups, full measurement invariance is confirmed, which supports the pooled data analysis. In contrast, if partial measurement invariance is not present, this absence can deflate the power of statistical testing, influence the accuracy of estimators, and provide misleading results in further analyses.
In the initial step, a significant difference must exist in the mediated effect for the groups by subtracting the mediated effect for Group A from that for Group B (i.e., $\left.d=p_{1}{ }^{(\mathrm{A})} \cdot p_{2}{ }^{(\mathrm{A})}-p_{1}{ }^{(\mathrm{B})} \cdot p_{2}{ }^{(\mathrm{B})}\right)$. This procedure can be performed using the MGA estimation approach to determine whether the mediated effect is significantly different, noting that the CoMe effect is present (Ryu \& Cheong, 2017). A p-value smaller than 0.05 suggests that the difference $d$ between the group-specific mediated effect is present. ${ }^{11}$ Thus the researcher can continue with comparing the mediated effects for each of the two groups (comparing the $\beta$ values of the mediated effect for Group A and Group B).

The comparison of the mediated effect for the two groups can be generated using any of the MGA techniques that we mentioned earlier (i.e., the permutation test, the non-parametric distance-based test, etc.). For example, if the mediated effect for the group with male individuals is large and significant $(\mathrm{Cl}$ 90 percent of the mediated effects exclude zero), whereas it is small and significant ( $\mathrm{Cl} 90$ percent of the mediated effects exclude zero) for the group with female individuals, one would then conclude that the mediated effect is significantly stronger (i.e., the magnitude of the relationship is greater) in the male group than in the female group. Alternatively, if the mediated effect for the group with male individuals is significant $(\mathrm{Cl} 90$ percent of the mediated effects exclude zero), but it is not significant for the group with female individuals, one would infer that the mediated effect only occurs in the male group but not in the female group. In other words, this approach allows the researcher to compare group differences in terms of the obtained significant mediated effect values. Finally, PLS-SEM researchers can compare the type of mediated effect such as full mediation versus partial mediation (complementary or competitive) (see Nitzl et al., 2016). 


\section{Assessment of Differences in Direct Effects to Determine the Type of Conditional Mediation Model}

CoMe analysis that draws on the interaction term approach (as per Sections 3.1-3.4) to examine hypotheses that concern the rate of change (i.e., slope) is based on an a priori specified type of CoMe model. However, the type of CoMe model must be determined a posteriori when conducting MGA to assess hypotheses that concern differences in the strength (i.e., magnitude) of relationships. Hence and as per Figure 1, it is necessary to identify whether a CoMe Model is of the $A, B$, or $C$ type, when carrying out MGA, in which the researcher analyzes whether $p_{1} \cdot p_{2}$ in Group 1 is different from that in Group 2. Based on the results and the presence of a significant difference in the two groups' mediated effects, the researcher can now observe whether $p_{1}$ is also significantly different between groups 1 and 2; thus, the researcher will find a Model $A$ type CoMe. If only $p_{2}$ is significantly different, the researcher will find a Model $B$ type CoMe. Lastly, if both $p_{1}$ and $p_{2}$ are significantly different, the result will indicate that the researcher has a Model $C$ type CoMe, where a particular categorical variable moderates both paths.

\section{Empirical Illustrations of Conditional Mediation Analyses in PLS-SEM}

In the following we describe how the conceptual fundamentals and guidelines embedded in Figure 1, and described above, are empirically applied in PLSSEM. In illustrating their application, we also refer to detailed step-by-step procedures for implementation in a PLS-SEM setting and outline these procedures in corresponding appendices to this paper.

The illustrative example of a CoMe model was extracted from the research model of Cheah et al. (2020b). The data were collected from 400 consumers who had experienced purchasing luxury products from a luxury retail store. The four main variables were modeled as composites: behavioral intention (BI), price image $(\mathrm{PI})$, perceived value $(\mathrm{PV})$, and sugrophobia (SUGRO). ${ }^{12} \mathrm{BI}$ was established as a composite estimated in Mode B, whereas PI, PV, and SUGRO were designed as a composite estimated in Mode A. Although their measurement models and their respective measurement modes were theoretically substantiated, to assess potential for any misspecification in measurement mode, confirmatory tetrad analysis (CTA-PLS) (Gudergan et al. 2008) was used (see Appendix A). The CTA-PLS assessment for $\mathrm{PI}, \mathrm{PV}$, and SUGRO revealed that all tetrads vanished, providing support for the employed reflective measurement mode. Conversely, the CTA-PLS assessment showed that several tetrads failed to vanish for $\mathrm{BI}$, implying that its measurement mode is indeed not reflective but rather formative. Importantly, the evaluation of the measurement models by using standard evaluation criteria (Hair et al., 2022) supports the measures' reliability and validity. This also holds for discriminant validity assessment using Henseler et al.'s (2015) heterotrait-monotrait (HTMT) criterion. Moreover, prior study has analyzed how PV positively mediates the relationship between $\mathrm{PI}$ and $\mathrm{BI}$. In addition, SUGRO is posited to negatively moderate the link between PI and PV (see Cheah et al., 2020).

Based on the abovementioned, we extend past findings by illustrating the CoMe analysis for three types of CoMe models (Models A, B, and C) and for two types of moderators, either using a continuous moderator or a categorical moderator that has been transformed from a continuous one. ${ }^{13}$ In regard to our empirical illustrations that draw on the transformation of a continuous moderator to a categorical moderator for CoMe analysis, we reiterate that researchers ought to be cautious in doing so because this transformation can produce a reduction in prediction power. Therefore, unless the researcher seeks to present findings in a particular way that serve a particular purpose (e.g., presentation of findings to a managerial audience to ease comprehension of actionable insights), or encounters data characterized by irregular or nonnormal distributions or outliers (DeCoster et al., 2009), estimations drawing on such transformed data ought to be avoided. Thus, the illustrations of the CoMe analysis for the three types of CoMe models (Models $\mathrm{A}, \mathrm{B}$, and $\mathrm{C}$ ) with the transformation of the moderator (SUGRO) to a categorical variable serve merely as empirical illustrations on how to establish both the CoMe index $(\omega)$ and CoMe effects (see Section 4.1) (14 $^{14}$ when explicitly desired by the researcher.

In drawing on the abovementioned research model of Cheah et al. (2020b), we first illustrate the CoMe analysis in a PLS-SEM setting to assess the rate of change (i.e., slope) in the mediated relationship(s) and focus on the following hypothesis: The indirect effect of the consumer's PI on his or her BI via PV decreases as SUGRO increases. Therefore, in Section 4.1, we illustrate CoMe analysis drawing on the interaction term approach to assess the conditional mediated relationship(s). Then, we demonstrate the CoMe analysis in a PLS-SEM setting to examine differences in strength (i.e., magnitude) in the conditional mediated relationship(s) and consider the following hypothesis: The indirect effect between PI and BI via $P V$ is more pronounced for the group of female consumers than for the group of male consumers. In doing so, we outline in Section 4.2 CoMe analysis employing the MGA approach. 


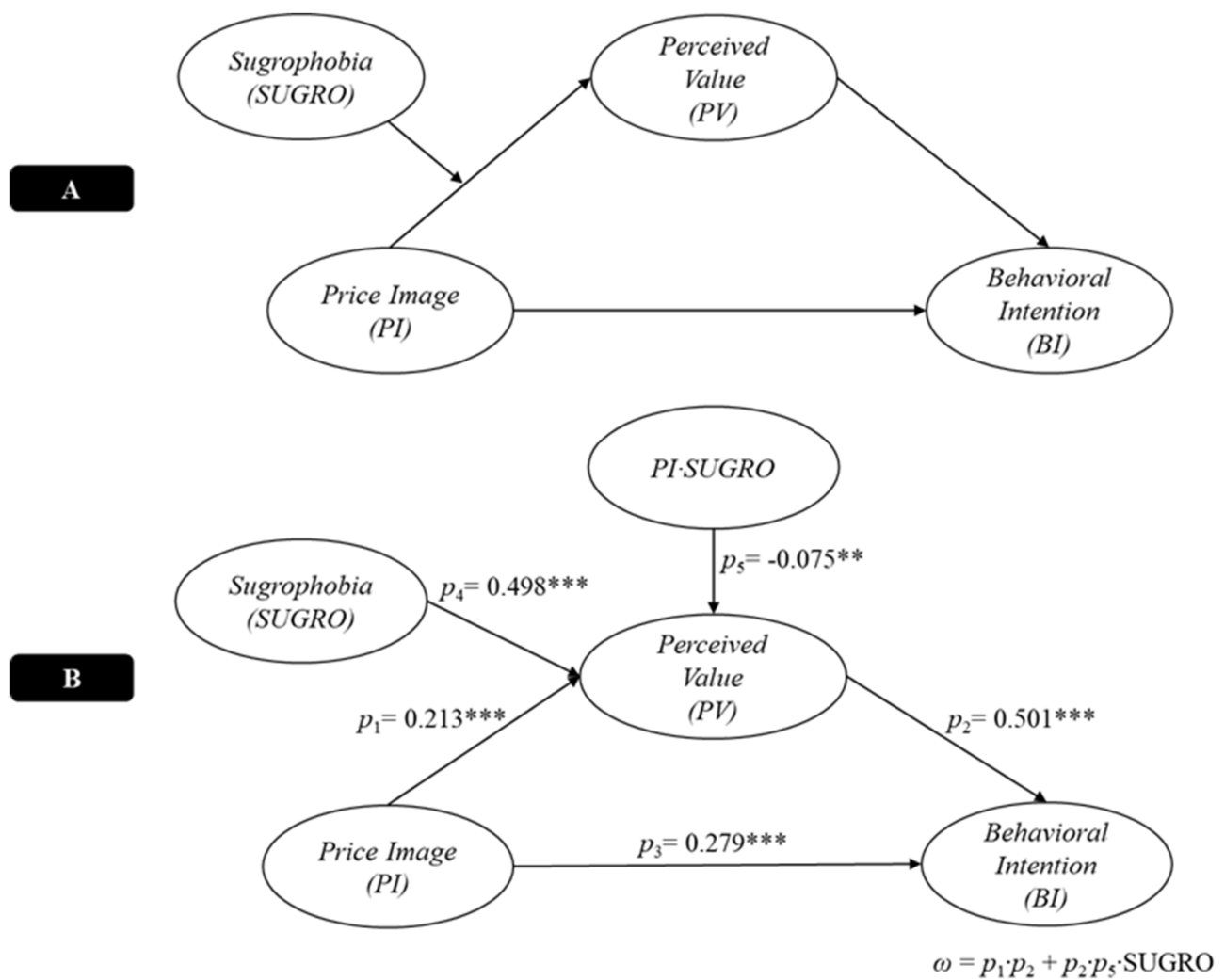

Note: SUGRO is treated as a continuous variable. $\quad * * * \mathrm{p}<0.001, * * \mathrm{p}<0.01$ (based on $\mathrm{t}(9999)$, one-tailed test)

C

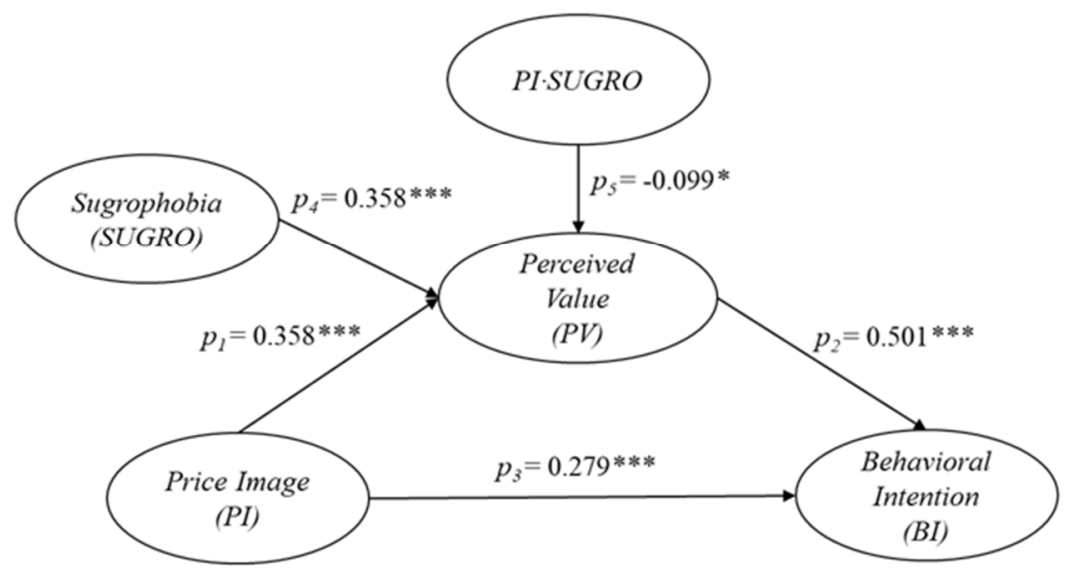

$\omega=p_{1} \cdot p_{2}+p_{2} \cdot p_{5} \cdot$ SUGRO

Note: SUGRO is treated as a categorical variable. $\quad * * * \mathrm{p}<0.001, * * \mathrm{p}<0.01$ (based on $\mathrm{t}(9999)$ ), one-tailed test)

Figure 9. Conceptual Model (Panel A), Structural Model Results with SUGRO as Continuous Variable (Panel B), and Structural Model Results with SUGRO as Categorical Variable (Panel C) for CoMe Model A

\section{Conditional Mediation Analysis- Interaction Term Approach}

\section{Conditional Mediation Analysis Model A- Continuous and Categorical Moderator}

For CoMe Model $A,{ }^{15}$ we focus on testing the influence of the moderator (SUGRO) on the mediated effect ( $p_{1}$ - $p_{2}$ ) (Figure 9). We first assess the CoMe index $(\omega)$, which is derived from the following equation: $\omega=p_{1}$. $p_{2}+p_{2} \cdot p_{5} \cdot$ SUGRO (Equation 1). Based on the estimation (Appendix B(i); see Figure B1, Panels A and $\mathrm{B}$ ), the result shows that " $p_{2} \cdot p_{5}$ " (or PI*SUGRO $>\mathrm{PV}->\mathrm{BI}$ ) for the use of the continuous moderator is supported, as the $\mathrm{Cl}$ excludes zero (90 percent $\mathrm{Cl}$ : -0.065 to -0.010 ). Similarly, the estimation (Appendix $B$ (ii); see Figure $B 7$, Panels $A$ and $B$ ) also shows that the result of " $p_{2} \cdot p_{5}$ " (or PI*SUGRO -> PV -> BI) for the 
use of the categorical moderator is supported, as the $\mathrm{Cl}$ excludes zero (90 percent $\mathrm{Cl}$ : -0.093 to -0.004 ). Since the index " $p_{2} \cdot p_{5}$ " of SUGRO is significant for both the continuous $(\beta=-0.037)$ and categorical $(\beta=$ -0.049) variable uses, we can conclude that the mediated effect of $\mathrm{PI}$ on $\mathrm{BI}$ through $\mathrm{PV}$ negatively depends on the moderator SUGRO (Table 2).

Then, we investigate the mediated effects at different levels of the moderator (Preacher et al., 2007) for both continuous and categorical moderators. Following the procedures derived from our guidelines (see Appendix $\mathrm{B}(\mathrm{i})$ ), we initially assess the SD of the moderator (SUGRO) when using the continuous moderator, as it will denote that the moderator is one SD below and one SD above the mean. ${ }^{16}$ To estimate the CoMe effects for low, medium, and high levels of the moderator, we use equations 6-8 for CoMe Model $A$ (see Appendix B(i), Figures B2-B5 for the details of the analysis). Results in Table 2 show that the path coefficients for the CoMe effects become weaker when the SUGRO effect is one SD above the mean. Additionally, the CoMe effects for low, medium, and high levels of the moderator are significant because both extremes of the $\mathrm{Cl}$ are positive. For the use of the categorical moderator, we illustrate this for the comparison between the low value (0) and the high value (1) (see Appendix B(ii), Figures B8-B11 for the details of the analysis). Moreover, the results in Table 2 show that the path coefficient for the CoMe effect for high SUGRO $(\beta=0.130)$ is weaker than for low SUGRO $(\beta=0.178)$, and both CoMe effects are

\section{A}

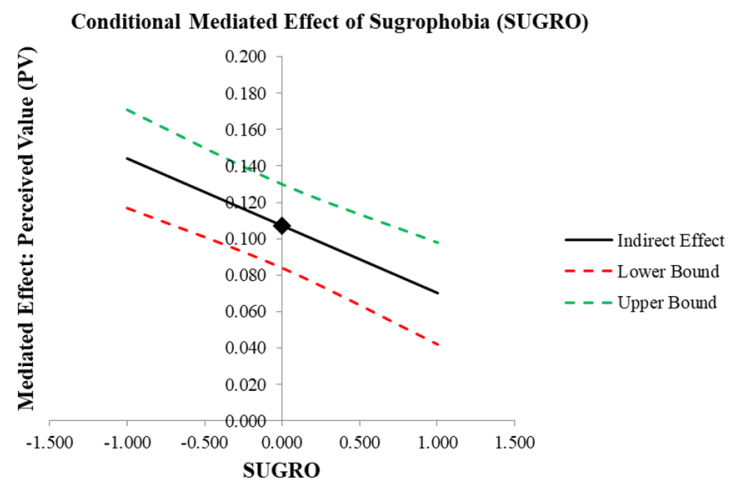

significant because both the extremes of the $\mathrm{Cl}$ are positive. Therefore, we find some evidence supporting the hypothesis in CoMe Model $A$ for both the categorical and continuous moderator use. That is, the indirect effect of $\mathrm{PI}$ on $\mathrm{BI}$ via $\mathrm{PV}$ decreases due to the negative impact of SUGRO (to corroborate this finding, also see Figure 10 that depicts the results using the Johnson-Neyman plot and simple slope for both the continuous and categorical moderators). ${ }^{17}$

\section{Conditional Mediation Analysis Model B- Continuous and Categorical Moderator}

For CoMe Model B, we focus on testing the influence of the moderator (SUGRO) on the mediated effect $\left(p_{1}\right.$ - $p_{2}$ ) (Figure 11). We first assess the CoMe index $(\omega)$, which is derived from the following equation: $\omega=p_{1} \cdot p_{2}$ $+p_{1} \cdot p_{5}$. SUGRO (Equation 2). Based on our estimations (see Appendix C(i); Figure C4), the results show that " $p_{1} \cdot p_{5}$ " for the use of the continuous moderator is not supported, as the $\mathrm{Cl}$ does include zero (90 percent $\mathrm{Cl}$ : -0.044 to 0.002). However, the estimations (see Appendix C(ii); Figure C11) show that the result of " $p_{1} \cdot p_{5}$ " for the use of the categorical moderator is supported, as the $\mathrm{Cl}$ does not include zero (90 percent $\mathrm{Cl}$ : -0.117 to -0.040$)$. The index " $p_{1}$ - $p_{5}$ " of SUGRO is significant for the categorical moderator $(\beta=-0.078)$; therefore, we can conclude that the mediated effect of $\mathrm{PI}$ on $\mathrm{BI}$ through $\mathrm{PV}$ decreases as the moderator SUGRO increases, but not when estimating with the continuous moderator ( $\beta$ $=-0.021)($ see Table 2$){ }^{18}$

\section{Figure 10. Continuous Variable (Panel A) and Categorical Variable (Panel B) for CoMe Effects Plot for}

CoMe Model A 


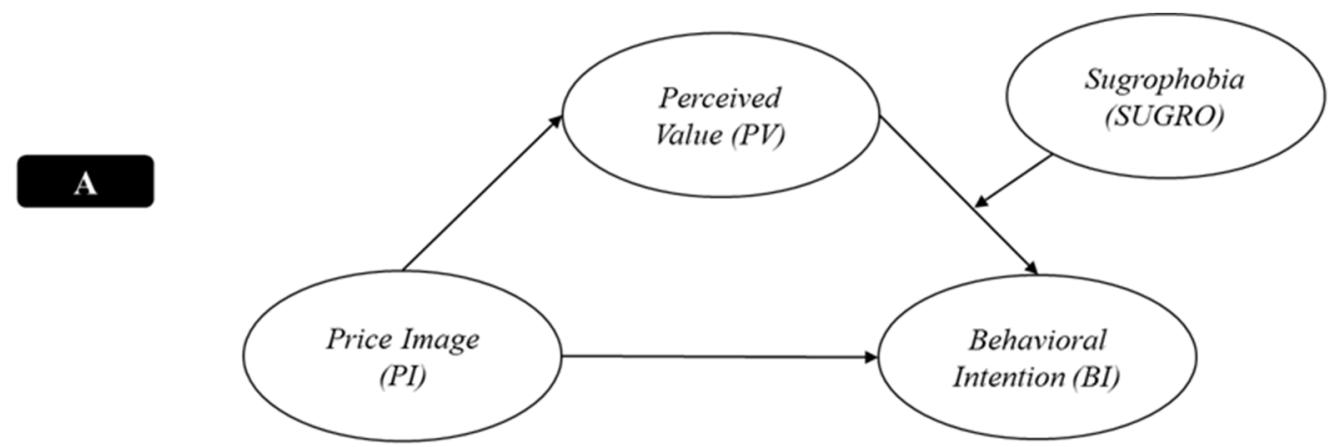

B

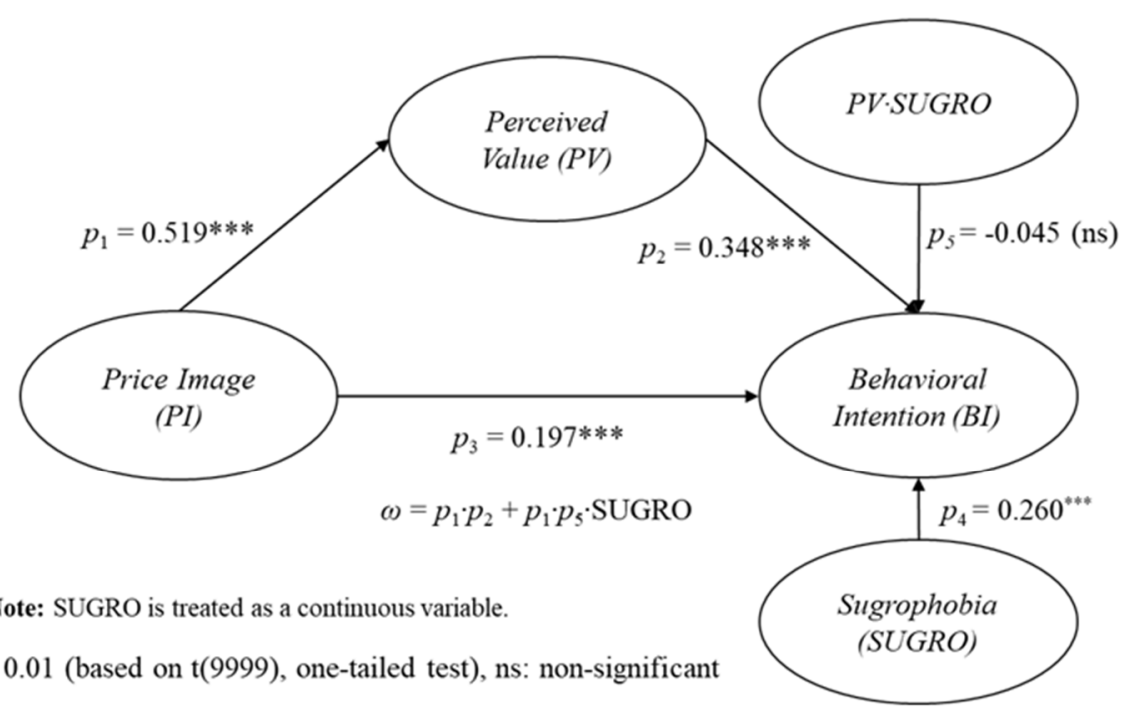

$* * * \mathrm{p}<0.001, * * \mathrm{p}<0.01$ (based on $\mathrm{t}(9999)$ ), one-tailed test), ns: non-significant

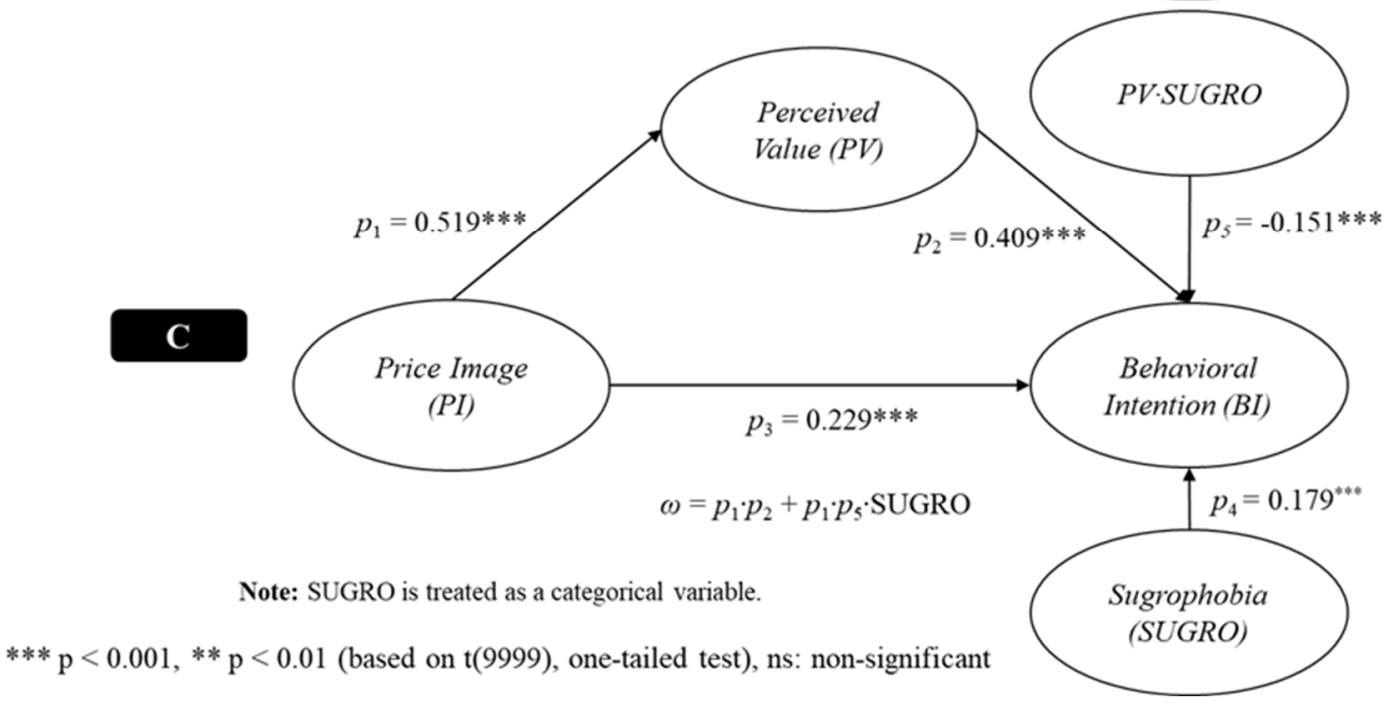

Figure 11. Conceptual Model (Panel A), Structural Model Results with SUGRO as Continuous Variable (Panel B), and Structural Model Results with SUGRO as Categorical Variable (Panel C) for CoMe Model B

Then, we investigate the mediated effects at different levels of the moderator (Preacher et al., 2007) for both the use of continuous and categorical moderators. Based on the procedures derived from our guidelines (please see details in Appendix C(i)), we initially assess the SD of the moderator (SUGRO) as continuous moderator, as it will denote that the moderator is one SD below and one SD above the mean. To estimate the CoMe effects for low, medium, and high moderator levels, we use equations 9-11 for CoMe Model $B$ (to conduct the analysis, also see Appendix C(i), Figures C5-C7). Results in Table 2 
show that the path coefficients of the CoMe effect become weaker when the SUGRO effect is one SD above the mean. ${ }^{19}$ Additionally, the CoMe effects for low, medium, and high moderator levels are significant because both extremes of the $\mathrm{Cl}$ are positive. However, we could not reveal whether a CoMe effect exists for the use of a continuous moderator because of the insignificant result of the CoMe index $(\omega)$. Meanwhile, the use as categorical moderator concerns the comparison between the low value $(0)$ and the high value (1) (for the analysis, also see Appendix C(ii), Figures C12-C14). Results in Table 2 show that the path coefficients of the CoMe effect for high SUGRO $(\beta=0.134)$ are weaker than for low SUGRO $(\beta=$ 0.212), and both CoMe effects are significant because both extremes of the $\mathrm{Cl}$ are positive. Therefore, we find some evidence supporting the hypothesis included in CoMe Model $B$ for the use of the categorical moderator only; that is, the mediated effect of $\mathrm{PI}$ on $\mathrm{BI}$ via PV reduces as SUGRO increases (to corroborate this finding, also see Figure 12 that depicts the results using the simple slope for categorical moderator).

\section{Conditional Mediation Analysis Model C- Continuous and Categorical Moderator}

Based on Figure 13 for CoMe Model C, we focus on testing the influence of the moderator (SUGRO) on the mediated effect $\left(p_{1} \cdot p_{2}\right)$. We first assess the CoMe index $(\omega)$, which is derived from the following equation: $\omega=p_{1} \cdot p_{2}+\left(p_{2} \cdot p_{5} \cdot p_{1} \cdot p_{7}\right)$ SUGRO $+p_{5} \cdot p_{7} \cdot$ SUGRO $^{2}$ (Equation 3). Based on our estimations (see Appendix $\mathrm{D}(\mathrm{i})$; Figure D4), the results show that " $p_{2} \cdot p_{5} \cdot+p_{1} \cdot p_{7}$ " for the continuous moderator is supported, as the $\mathrm{Cl}$ does not include zero (90 percent $\mathrm{Cl}$ : -0.057 to -0.013). Similarly, our estimations (see Appendix D(ii); Figure D13) show that the result of " $p_{2} \cdot p_{5} \cdot+p_{1} \cdot p_{7}$ " for the categorical moderator is also supported, as the $\mathrm{Cl}$ excludes zero (90 percent $\mathrm{Cl}$ : -0.137 to -0.049 ). ${ }^{20}$
Moreover, the index " $p_{2} \cdot p_{5} \cdot+p_{1} \cdot p_{7}$ " of SUGRO is significant for both the continuous $(\beta=-0.035)$ and categorical $(\beta=-0.093)$ moderators. Therefore, we can conclude that the mediated effect of $\mathrm{PI}$ on $\mathrm{BI}$ through PV decreases as the moderator SUGRO increases (Table 2).

Then, we investigate the mediated effects at different levels of the moderator (Preacher et al., 2007) for the use of both continuous and categorical moderators. Again, based on the procedures derived from our guidelines (see Appendix $\mathrm{D}(\mathrm{i})$ ), we initially assess the SD of the moderator (SUGRO) for continuous moderator, as it will denote that the moderator is one SD below and one SD above the mean. ${ }^{21}$ To estimate the CoMe effects for low, medium, and high moderator levels, we use equations 12-14 for Model C (see Appendix D(i), Figures D5-D10). Results in Table 2 show that the path coefficients of the CoMe effect become weaker when the SUGRO effect is one SD above the mean. Additionally, the CoMe effects for low, medium, and high levels of the moderator are significant because both extremes of the $\mathrm{Cl}$ are positive. Meanwhile, using the categorical moderator concerns the comparison between the low value (0) and the high value (1) (for the analysis, also see Appendix D(ii), Figures D14-D15). Results in Table 2 reveal that the path coefficients of the CoMe effect for high SUGRO $(\beta=0.067)$ are weaker than for low SUGRO ( $\beta=0.146)$, and both CoMe effects are significant because both extremes of the $\mathrm{Cl}$ are positive. Therefore, we find some evidence supporting the hypothesis in CoMe Model $C$ for using both the categorical and continuous moderators. That is, the mediated effect of PI on $\mathrm{BI}$ via $\mathrm{PV}$ decreases as the moderator SUGRO increases (to corroborate this finding, also see Figure 14 that depicts the results using the Johnson-Neyman plot and simple slope for the use of both the continuous and categorical moderators).

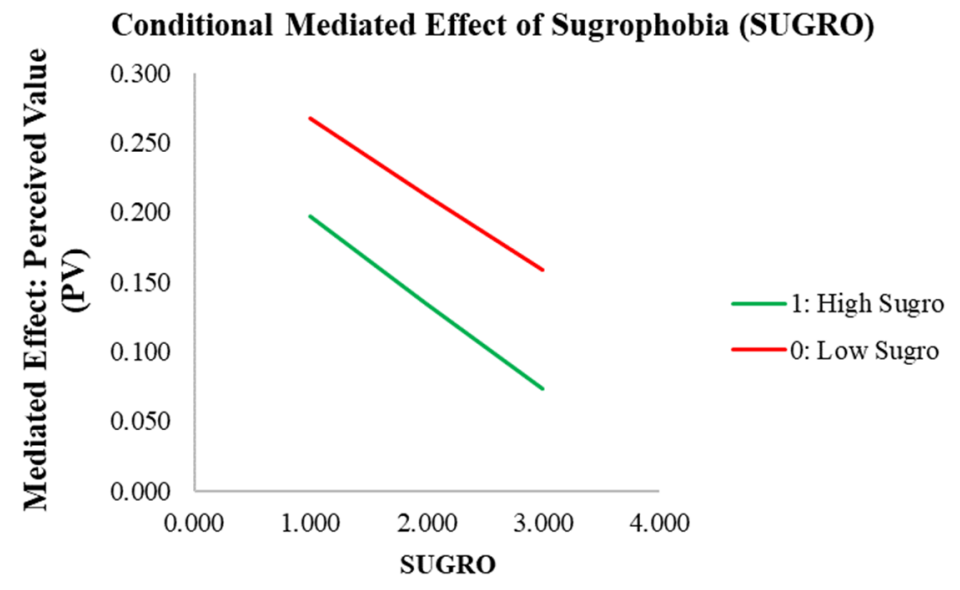

Figure 12. Categorical Variable for CoMe Effects Plot for CoMe Model B 

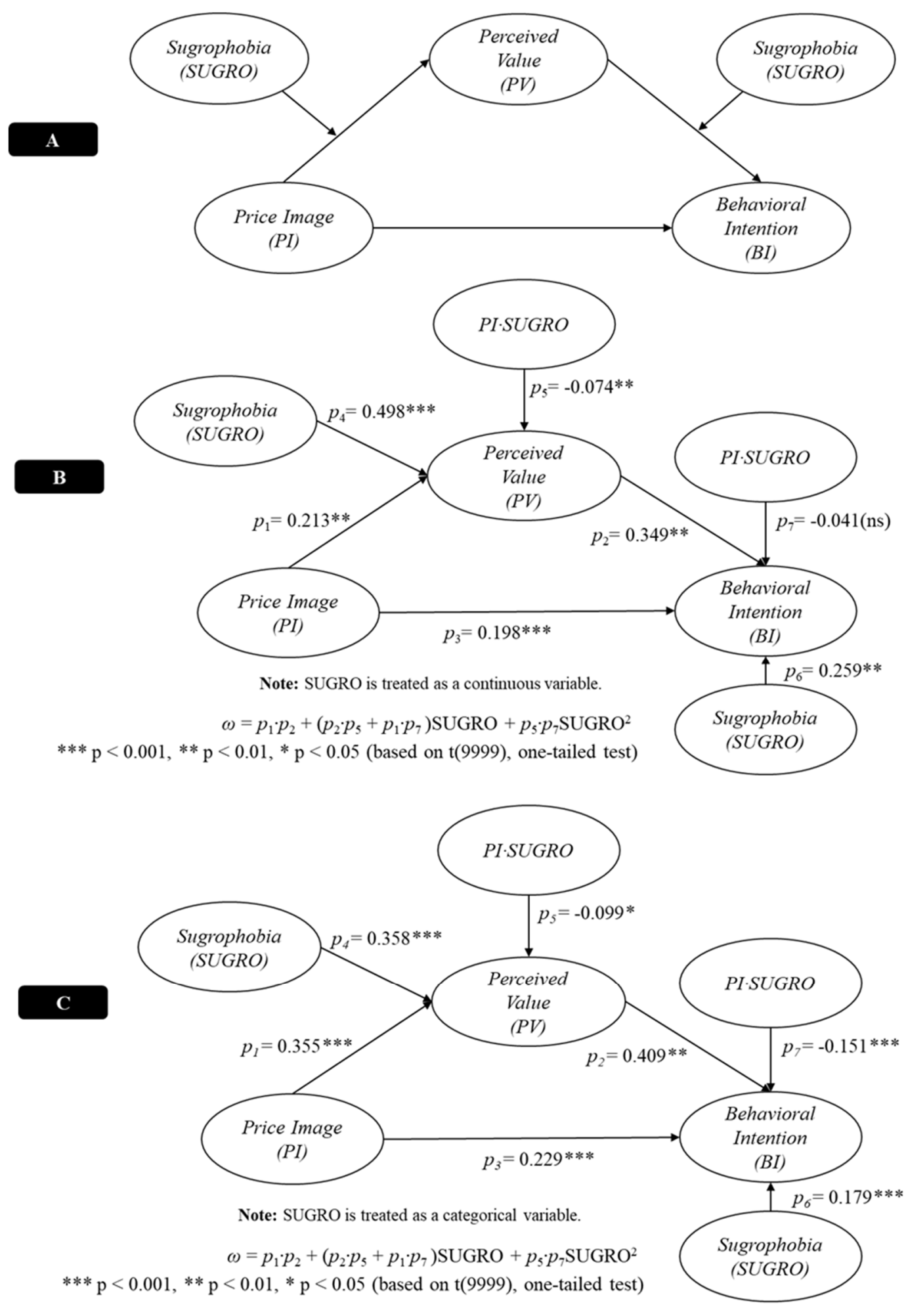

Figure 13. Conceptual Model (Panel A), Structural Model Results with SUGRO as Continuous Variable (Panel B), Structural Model Results with SUGRO as Categorical Variable (Panel C) for CoMe Model C 

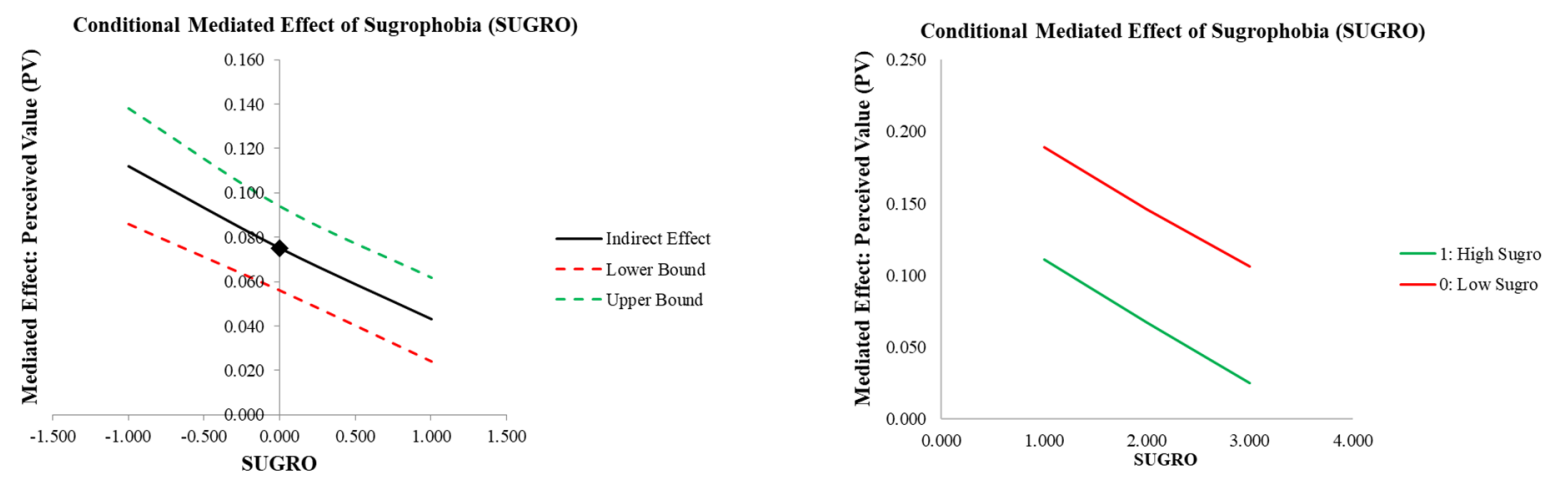

Figure 14. Continuous Variable (Panel A) and Categorical Variable (Panel B) for CoMe Effects Plot for CoMe Model C

Table 2. Conditional Mediated Effect Results for CoMe Models A-C (Continuous Data \& Transformation from Continuous Data to Median Split)

\begin{tabular}{|c|c|c|c|c|c|c|c|}
\hline Model & Moderator Type & $\begin{array}{l}\text { Conditional mediated effect } \\
\text { (CoMe effect) }\end{array}$ & Std Beta & $\begin{array}{c}\text { Lower } \\
\text { Percentile } \\
(5 \%)\end{array}$ & $\begin{array}{c}\text { Upper } \\
\text { Percentile } \\
(95 \%)\end{array}$ & SE & t-value \\
\hline \multirow[t]{9}{*}{ A } & \multirow{5}{*}{ Continuous } & Index of Conditional Mediation & -0.037 & -0.064 & -0.011 & 0.016 & 2.295 \\
\hline & & Sugrophobia (W) & & & & & \\
\hline & & Low $(-1.0013)$ & 0.144 & 0.101 & 0.189 & 0.027 & 5.378 \\
\hline & & Medium $(0.0000)$ & 0.107 & 0.071 & 0.144 & 0.023 & 4.770 \\
\hline & & High (1.0013) & 0.070 & 0.023 & 0.116 & 0.028 & 2.474 \\
\hline & \multirow[t]{4}{*}{$\begin{array}{l}\text { Dichotomous } \\
\text { (Median Split) }\end{array}$} & $\begin{array}{l}\text { Index of Conditional Mediation- } \\
\text { Dummy Variable }\end{array}$ & -0.049 & -0.093 & -0.003 & 0.027 & 1.798 \\
\hline & & Sugrophobia (W) & & & & & \\
\hline & & Low $(0)$ & 0.178 & 0.132 & 0.227 & 0.029 & 6.159 \\
\hline & & High (1) & 0.130 & 0.071 & 0.196 & 0.038 & 3.406 \\
\hline \multirow[t]{9}{*}{ B } & \multirow[t]{5}{*}{ Continuous } & Index of Conditional Mediation & -0.021 & -0.044 & 0.002 & 0.014 & 1.518 \\
\hline & & Sugrophobia (W) & & & & & \\
\hline & & Low $(-1.0011)$ & 0.201 & 0.144 & 0.261 & 0.036 & 5.657 \\
\hline & & Medium $(0.0000)$ & 0.180 & 0.128 & 0.236 & 0.033 & 5.502 \\
\hline & & High (1.0011) & 0.159 & 0.103 & 0.220 & 0.036 & 4.465 \\
\hline & \multirow[t]{4}{*}{$\begin{array}{l}\text { Dichotomous } \\
\text { (Median Split) }\end{array}$} & $\begin{array}{l}\text { Index of Conditional Mediation- } \\
\text { Dummy Variable }\end{array}$ & -0.078 & -0.117 & -0.040 & 0.024 & 3.294 \\
\hline & & Sugrophobia (W) & & & & & \\
\hline & & Low $(0)$ & 0.212 & 0.159 & 0.267 & 0.033 & 6.504 \\
\hline & & High (1) & 0.134 & 0.073 & 0.197 & 0.038 & 3.559 \\
\hline \multirow[t]{9}{*}{ C } & \multirow[t]{5}{*}{ Continuous } & Index of Conditional Mediation & -0.035 & -0.057 & -0.013 & 0.013 & 2.658 \\
\hline & & Sugrophobia (W) & & & & & \\
\hline & & Low (-1.0012) & 0.112 & 0.073 & 0.157 & 0.026 & 4.378 \\
\hline & & Medium $(0.0000)$ & 0.075 & 0.047 & 0.107 & 0.019 & 4.035 \\
\hline & & High (1.0012) & 0.043 & 0.013 & 0.076 & 0.019 & 2.227 \\
\hline & \multirow[t]{4}{*}{$\begin{array}{l}\text { Dichotomous } \\
\text { (Median Split) }\end{array}$} & $\begin{array}{l}\text { Index of Conditional Mediation- } \\
\text { Dummy Variable }\end{array}$ & -0.093 & -0.137 & -0.048 & 0.027 & 3.443 \\
\hline & & Sugrophobia (W) & & & & & \\
\hline & & Low $(0)$ & 0.146 & 0.106 & 0.189 & 0.026 & 5.658 \\
\hline & & High (1) & 0.067 & 0.031 & 0.111 & 0.025 & 2.699 \\
\hline
\end{tabular}




\section{Comparison of Prediction Ability between Continuous and Categorical Moderators}

We extend the evaluation assessment of CoMe effects drawing on the interaction term approach by comparing the result of in-sample prediction (i.e., coefficient of determination, adjusted coefficient of determination, and model selection criteria) and outof-sample prediction (i.e., blindfolding and PLS_predict). ${ }^{22}$ This allows assessment of the efficacy of various quality criteria between the use of a continuous moderator and the transformation from continuous to categorical moderator. Results in Table 3 exhibit that the majority of the criteria for in-sample and out-of-sample predictions favor the use of a continuous moderator compared with the use of transformation from continuous to the categorical moderator.

To reiterate, unless the researcher seeks to derive and present findings in a way that they might serve a particular purpose (e.g., presentation of findings to a managerial audience to ease comprehension of actionable insights) or encounters data characterized by irregular or non-normal distributions or outliers (DeCoster et al., 2009), when a continuous moderator is suitable and available in CoMe analysis, we recommend researchers to be very cautious in transforming continuous data to categorical one for CoMe analysis because it loses prediction power. In the case that the researcher chooses to work with data that are transformed to categorical one, all CoMe analyses ought to be based on sufficient statistical power to warrant producing consequential results and drawing meaningful conclusions.

\section{Conditional Mediation Analysis-MGA Approach Using Categorical Moderator}

In this section, we continue drawing on the data from Cheah et al. (2020) to illustrate the use of MGA in CoMe analysis, especially when the moderator is categorical and the research aim is testing the difference in strength (magnitude) between mediated effects. Hence, we re-estimate the model by replacing SUGRO with gender as the categorical moderator. ${ }^{23}$ As outlined above, the three constructs (PI and PV) are modeled as composites and estimated in Mode A, while $\mathrm{Bl}$ is modeled as a composite and estimated in Mode B. Additionally, since gender is used as a potential moderator, the total of 400 consumers sample is split into two groups: female $(n=206)$ and male consumers $(n=194)$ (Figure 15, Panel A).

In this illustration, we evaluate whether the mediated effect of $p_{1} \cdot p_{2}$ for the female group is significantly different from that for the male group. ${ }^{24}$ The equation for this significant difference is $d=p_{1}$ (Female) $\cdot p_{2}$ (Female) $p_{1}{ }^{\text {(Male) }} \cdot p_{2}$ (Male). Thus, an MGA is carried out. After following the MICOM procedure and concluding that partial measurement invariance is established, we apply a permutation-based test (Chin \& Dibbern, 2010), representing the most powerful nonparametric MGA testing approach (Klessel et al., 2021). ${ }^{25}$

Table 3. Causal-Prediction Criteria Values for CoMe Models A-C

\begin{tabular}{|c|c|c|c|c|c|c|c|c|c|c|c|}
\hline \multicolumn{4}{|c|}{ R2/Adj R² } & \multicolumn{3}{|c|}{ Blindfolding Q ${ }^{2}$} & \multicolumn{2}{|c|}{ PLS_predict } & \multicolumn{3}{|c|}{ Model Selection Criteria } \\
\hline $\begin{array}{l}\text { CoMe } \\
\text { Model }\end{array}$ & $\begin{array}{c}\text { Moderator } \\
\text { Type }\end{array}$ & PV & $\mathrm{BI}$ & PV & $\mathrm{BI}$ & $\begin{array}{l}\text { RMSE } \\
\text { (PV/BI) }\end{array}$ & $\begin{array}{c}\text { MAE } \\
\text { (PV/BI) }\end{array}$ & $\begin{array}{c}\mathrm{Q}^{2} \text { _predict } \\
\text { (PV/BI) }\end{array}$ & $\begin{array}{c}\text { AIC } \\
\text { (PV/BI) }\end{array}$ & $\begin{array}{c}\text { BIC } \\
\text { (PV/BI) }\end{array}$ & $\begin{array}{c}\text { GM } \\
\text { (PV/BI) }\end{array}$ \\
\hline A & Continuous & $\begin{array}{l}0.475 / \\
0.471\end{array}$ & $\begin{array}{l}0.474 / \\
0.471\end{array}$ & 0.382 & 0.424 & $\begin{array}{l}0.738 / \\
0.775\end{array}$ & $\begin{array}{l}0.588 / \\
0.595\end{array}$ & $\begin{array}{l}0.466 / \\
0.409\end{array}$ & $\begin{array}{l}-251.063 / \\
-251.895\end{array}$ & $\begin{array}{c}235.097 / \\
-239.920\end{array}$ & $\begin{array}{l}422.966 / \\
416.974\end{array}$ \\
\hline & \begin{tabular}{|c} 
Dichotomous \\
(Median \\
Split)
\end{tabular} & $\begin{array}{l}0.380 / \\
0.376\end{array}$ & $\begin{array}{l}0.474 / \\
0.471\end{array}$ & 0.305 & 0.423 & $\begin{array}{l}0.802 / \\
0.808\end{array}$ & $\begin{array}{l}0.633 / \\
0.615\end{array}$ & $\begin{array}{l}0.368 / \\
0.357\end{array}$ & $\begin{array}{c}184.344 / \\
-251.773\end{array}$ & $\begin{array}{l}-168.378 / \\
-239.799\end{array}$ & $\begin{array}{l}422.966 / \\
416.974\end{array}$ \\
\hline$B$ & Continuous & $\begin{array}{l}0.270 / \\
0.268\end{array}$ & $\begin{array}{l}0.516 / \\
0.512\end{array}$ & 0.217 & 0.458 & $\begin{array}{l}0.865 / \\
0.773\end{array}$ & $\begin{array}{l}0.682 / \\
0.594\end{array}$ & $\begin{array}{l}0.261 / \\
0.412\end{array}$ & $\begin{array}{r}122.741 / \\
-281.632 \\
\end{array}$ & $\begin{array}{l}-114.758 / \\
-261.675\end{array}$ & $\begin{array}{l}410.983 / \\
428.957\end{array}$ \\
\hline & \begin{tabular}{|c} 
Dichotomous \\
(Median \\
Split)
\end{tabular} & $\begin{array}{l}0.270 / \\
0.268\end{array}$ & $\begin{array}{l}0.508 / \\
0.503\end{array}$ & 0.217 & 0.447 & $\begin{array}{l}0.867 / \\
0.796\end{array}$ & $\begin{array}{l}0.680 / \\
0.618\end{array}$ & $\begin{array}{l}0.261 / \\
0.376\end{array}$ & $\begin{array}{l}-122.737 / \\
-274.957\end{array}$ & $\begin{array}{l}114.754 / \\
-254.999\end{array}$ & $\begin{array}{l}410.983 / \\
428.957\end{array}$ \\
\hline$C$ & Continuous & $\begin{array}{l}0.475 / \\
0.471\end{array}$ & $\begin{array}{l}0.516 / \\
0.511\end{array}$ & 0.382 & 0.458 & $\begin{array}{l}0.737 / \\
0.754\end{array}$ & $\begin{array}{l}0.588 / \\
0.569\end{array}$ & $\begin{array}{l}0.465 / \\
0.455\end{array}$ & $\begin{array}{l}-250.831 / \\
-281.444\end{array}$ & $\begin{array}{l}-234.866 / \\
-261.486\end{array}$ & $\begin{array}{l}422.966 / \\
428.957\end{array}$ \\
\hline & \begin{tabular}{|c} 
Dichotomous \\
(Median \\
Split)
\end{tabular} & $\begin{array}{l}0.380 / \\
0.375\end{array}$ & $\begin{array}{l}0.508 / \\
0.503\end{array}$ & 0.305 & 0.447 & $\begin{array}{l}0.801 / \\
0.783\end{array}$ & $\begin{array}{l}0.633 / \\
0.597\end{array}$ & $\begin{array}{l}0.367 / \\
0.397\end{array}$ & $\begin{array}{l}-184.321 / \\
-274.952\end{array}$ & $\begin{array}{l}-168.355 / \\
-254.995\end{array}$ & $\begin{array}{l}422.966 / \\
428.957\end{array}$ \\
\hline
\end{tabular}

Note: Gray shading represents the best value 


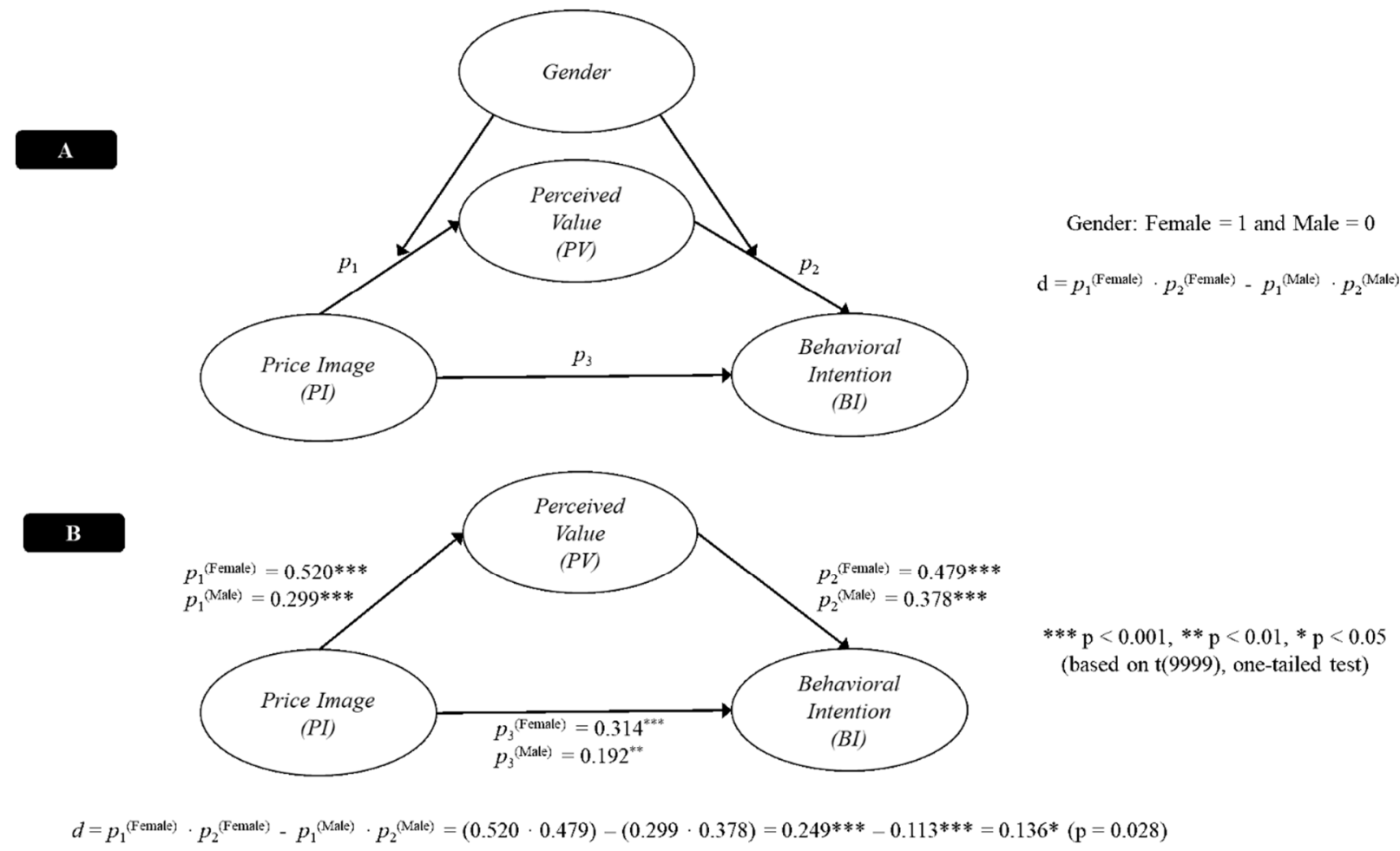

Figure 15. Conceptual Model (Panel A) and Structural Model Results (Panel B) with Categorical Moderator

Table 4. The Difference in Mediated Effects Between Female and Male Consumers

\begin{tabular}{|c|c|c|c|c|c|c|c|c|c|c|}
\hline \multirow{2}{*}{\begin{tabular}{|l} 
Gender \\
Mediated Effect \\
\end{tabular}} & \multicolumn{4}{|c|}{ Female $(n=206)$} & \multicolumn{4}{|c|}{ Male $(n=194)$} & \multicolumn{2}{|r|}{ Male-Female } \\
\hline & $\beta$ & $t$-value & $p$-value & PBCI $90 \%$ & $\beta$ & $t$-value & $p$-value & PBCI $90 \%$ & $d$ & Permutation $p$-value \\
\hline $\mathrm{PI}->\mathrm{PV}->\mathrm{BI}\left(p_{1} \cdot p_{2}\right)$ & 0.249 & 5.317 & 0.000 & {$[0.172 ; 0.328]$} & 0.113 & 3.355 & 0.000 & {$[0.062 ; 0.170]$} & 0.136 & 0.028 \\
\hline Direct Effect & $\beta$ & $t$-value & $p$-value & PBCI $90 \%$ & $\beta$ & $t$-value & $p$-value & PBCI $90 \%$ & $d$ & Permutation $p$-value \\
\hline $\mathrm{PI}$-> PV $\left(p_{1}\right)$ & 0.520 & 8.169 & 0.000 & {$[0.399 ; 0.611]$} & 0.299 & 4.305 & 0.000 & {$[0.174 ; 0.403]$} & 0.220 & 0.013 \\
\hline $\mathrm{PV}->\mathrm{BI}\left(p_{2}\right)$ & 0.479 & 6.691 & 0.000 & {$[0.357 ; 0.586]$} & 0.378 & 5.149 & 0.000 & {$[0.248 ; 0.493]$} & 0.102 & 0.164 \\
\hline $\mathrm{PI}$-> BI $\left(p_{3}\right)$ & 0.314 & 4.624 & 0.000 & {$[0.204 ; 0.426]$} & 0.192 & 2.370 & 0.009 & {$[0.044 ; 0.312]$} & 0.122 & 0.103 \\
\hline
\end{tabular}

Note: Gray shading represents significant value.

The permutation-based test shows how the difference in mediated effects between the female and male groups is significantly different from zero (Table 4). Therefore, the mediated effect of PI on BI through PV is conditional on gender as the moderator. Although we observe partial mediation in both groups (see the results in Figure 15, Panel $B$ and Table 4), the mediated effect is significantly higher for the female group $(\beta=0.249)$ than for the male group $(\beta=0.113) .{ }^{26}$ Therefore, we find support for a significant CoMe effect. ${ }^{27}$ Finally, given that the study shows a significant difference only between $p_{1}$ (Female) and $p_{1}{ }^{\text {(Male) }}$, the model described here corresponds to a CoMe Model A (Table 4).

\section{Summary and Conclusions}

CoMe analysis often opens up new avenues for analyzing new research questions. Such modeling provides deeper insights into the intricacies of processes or under which conditions they occur, which is not the case when mediation and moderation are tested independently. However, the use and application of CoMe analysis in PLS-SEM have been scarce and, in many cases, vague in their explanation. One reason could be the lack of experience and knowledge to handle such complexities in a PLS-SEM setting, especially when the estimation procedure is not straightforward. With these issues in mind, this paper illuminates conceptual fundamentals and develops guidelines for CoMe analysis within the PLSSEM context. In doing so, clear distinction between the different types of CoMe models (i.e., Models A-E) is made. Furthermore, the paper outlines the illustrative use of CoMe analysis in PLS-SEM and presents detailed step-by-step procedures to estimate and assess CoMe effects in the PLS-SEM setting (as per corresponding appendices). In doing so, we explain 
how to estimate the CoMe index $(\omega)$, and illustrate the applications of CoMe analysis in PLS-SEM for the most prominent CoMe models (i.e., Models A, B, and C). Such detailed illustrations will increase researchers' awareness of the procedures for and serve as a guide for estimating and interpreting CoMe results in PLS-SEM use. Thus, this paper responds to the recent claim by Sarstedt et al. (2020a) about the unnecessity of using PLS-SEM and PROCESS in tandem.

To recap the utility of conducting CoMe analysis in PLS-SEM, we provide three concluding recommendations concerning model specification, causal inferences, and issues regarding sample size. Although some of these recommendations apply to all research areas, they are particularly crucial for proficiently conducting CoMe analysis. First, a clear and robust theoretical basis must be established for any CoMe model (i.e., first-, second-, or first- and second-stage moderation) (Borau et al., 2015). Holland et al. (2017) have stressed the importance of theoretical rigor to substantiate the correct CoMe model and whether hypothesized arguments concern the rate of change (i.e., slope) or differences in the strength (i.e., magnitude) of mediated relationships that are conditional. The guidelines and procedures provided here, regardless of how robust and effective they are, in no way undermine the central importance of a theoretical foundation on which a CoMe model ought to rest. In other words, each moderated path in a mediation model must be founded and rooted clearly in a theoretical framework (see Holland et al., 2017). The same logic applies when multiple or serial mediation relationships are moderated in a model: each moderation must be justified. Hence, the approach to conducting CoMe analysis rests on sound theoretical substantiation and suitable data from a fitting research design.

Second, CoMe analysis implies sequential causation, which may occur simultaneously but can also happen over time, where changes in some variables affect changes in other variables over various points in time (Holland et al., 2017). Therefore, drawing on data from experimental and longitudinal research designs is often appropriate (see Mathieu et al., 2008; Roemer, 2016; Stone-Romero \& Rosopa, 2011; Streukens \& Leroi-Werelds, 2016b). However, this does not render the use of data from cross-sectional research designs useless when conducting CoMe analysis. Despite the limitations of such research designs (i.e., omitted variable problems that can cause spurious relationships), CoMe analysis resting on such research designs still allows drawing inferences about the direct and mediated effects and differences in the rate of change (i.e., slope) or in the strength (i.e., magnitude) in relationships produced by moderation.
Thus, one might use the approach provided by Hult et al. (2018) to assess and control endogeneity, particularly in the case of omitted variables (Sarstedt et al., 2020b; Spector, 2019), to conclude a causal effect from the CoMe effect estimation in PLS-SEM. Furthermore, when theoretical reasoning requires constructs being measured at certain points in time, such should be considered in a cross-sectional research design underlying the data when conducting CoMe analysis.

Third, when researchers seek to conduct MGA to detect CoMe effects, sufficient statistical power must be given to avoid type II errors (Dawson, 2014; Hayes, 2017). Researchers must ensure that the split samples for each group have close to equal and sufficient sample sizes to achieve sufficient statistical power (Aguinis et al., 2017). Likewise, if researchers choose to work with categorial data that are transformed from continuous one, all CoMe analyses ought to be based on sufficient statistical power to warrant producing consequential results. They can rely on power analyses that consider the model structure, the anticipated significance level, and the expected effect sizes (e.g., Marcoulides \& Chin, 2013). Alternatively, Hair et al. (2022) have provided power tables that indicate the required sample sizes for various measurement and structural model constellations. Finally, Kock and Hadaya (2018) suggested the inverse square root method and the gamma exponential method as two new approaches for minimum sample size calculations assess whether sufficient statistical power exists.

Overall, we hope this paper, together with the detail provided in the appendices, will increase the number of studies applying CoMe analysis in PLS-SEM proficiently. Although our paper offers guidance for carrying out CoMe analysis in PLS-SEM, future research should seek to extend the detail provided here. For example, researchers could examine CoMe analysis for different types of CoMe models such as CoMe Model D or CoMe Model E that we had excluded in this paper but also others such as one in which two exogenous moderators (rather than one) simultaneously condition either the first-stage or second-stage of the mediated relationship.

Additionally, researchers could explore the efficacy of the PLS algorithm when estimating the CoMe effect against other composite-based SEM methods, such as consistent PLS (Dijkstra \& Henseler, 2015a; 2015b), generalized structured component analysis (Hwang et al., 2010), and regularized canonical correlation analysis (Tenenhaus \& Tenenhaus, 2011), with the adaptation of sampling weight adjustments in PLSSEM (Becker \& Ismail, 2016; Cheah et al., 2020a). Moreover, researchers could explore consideration of more complex CoMe research frameworks, such as 
how a moderator influences parallel or serial mediating models simultaneously (Hayes, 2017).

In addition, a further interesting direction for future research is to examine how best to incorporate CoMe analyses into repeated-measures designs (experimental designs) given that repeated-measures data allow scholars to target statistical inference at between-subject and within-subject sources of variation (Curran \& Bauer, 2011; Streukens \& LeroiWerelds, 2016b). Similarly, assessments of CoMe models that involve intricate research designs, such as multilevel modeling (Hwang et al., 2007) and longitudinal data (Roemer, 2016), although in their early stages of development, require further advancement within the PLS-SEM context, in general, and when assessing CoMe models, in particular.

Also, whereas the approaches to CoMe analysis presented and outlined in this paper concern a priori, theoretically derived determination of CoMe models and considerations of whether moderations concern the rate of change (i.e., slope) versus differences in the strength (i.e., magnitude) of mediated relationships, the guidance presented here can be

\section{Notes}

${ }^{1}$ The illustrative uses of the CoMe index $(\omega)$ for CoMe Model A, CoMe Model and CoMe Model C are explained in Section 4.1. As for the CoMe index $(\omega)$ for CoMe Model D and CoMe Model E, the present study does not illustrate the empirical procedures but researchers could refer to Hayes (2015) or contact the corresponding author.

${ }^{2}$ A "conditional direct effect" occurs when the direct effect of $X$ on $Y$ estimates how differences in $X$ relate to differences in $Y$ holding constant the proposed mediator (Hayes, 2017). In other words, the conditional direct effect quantifies the effect of $X$ on $Y$ independent of the mediator but conditioned on a value of a moderator.

3 To obtain the bootstrap confidence intervals, in line with Aguirre-Urreta and Rönkkö (2018), "researchers should generally use the percentile method. However, when the reliability coefficient's bootstrap distribution is skewed, the bias corrected and accelerated $(\mathrm{BCa})$ bootstrap interval method" should be used (p. 9).

${ }^{4}$ If using existing data-especially continuous ones-that do not align with requirements of the analysis goal (i.e., researchers may have hypothesized that the first- and/or second-stage paths in a mediated relationship differ in terms of strengths/magnitude or directionality), researchers could subsequently transform their data into categorical data to test the CoMe effect.

${ }^{5}$ Researchers need to be very cautious when converting continuous variables to categorical variables (also known as artificially dichotomizing) for testing a CoMe model. Doing so can reduce the statistical power to detect a CoMe effect and can produce a downward bias in the size of estimated CoMe effects (Aguinis et al., 2017). Additionally, some researchers also throw up theoretical questions about what particular dividing points (i.e., mean, median, and percentile) should be used (Hayes \& Preacher, 2014). Hence, researchers expanded to also incorporate exploratory approaches that draw on the assessment of unobserved heterogeneity. For instance, approaches such as FIMIX-PLS in combination with, for example, PLSPOS, PLS-GAS, or PLS-IRRS (e.g., Ratzmann, Gudergan, \& Bouncken, 2016; Sarstedt, Ringle, \& Gudergan, 2016; Sarstedt, Ringle, \& Hair, 2017) can be applied in a CoMe analysis context, especially when assessing differences in the strength (i.e., magnitude) of certain paths.

Finally, although we advocate rigorous theoretical substantiation for any CoMe model, another direction for future research concerns developing approaches for model selection among different CoMe model types (e.g., Models A-B). Such advances can build on using the effectiveness of various quality criteria available in PLS-SEM, such as the model fit (Dijkstra \& Henseler, 2015a, 2015b), PLS_predict (Shmueli et al., 2016; Shmueli et al., 2019), cross-validated predictive ability testing (Liengaard et al., 2020), and model selection criteria (Danks et al. 2020; Sharma et al., 2019; Sharma et al., 2020).

ought to exercise caution unless converting a continuous variable to a categorical variable has strong underlying reasoning and justification. Despite these well-known problems, dichotomizing from a continuous variable is frequently used by experimental consumer researchers. Furthermore, some of the benefits surrounding the continued use of dichotomization are: (1) it produces meaningful findings that are easy to understand for a wider, possibly less technically knowable, audience (i.e., practitioners), and (2) it allows dealing with irregular or nonnormal distributions, or the presence of outliers (DeCoster et al., 2009).

6 By default, the estimation of reflectively specified constructs draws on Mode A, whereas PLS-SEM uses Mode B for formatively specified constructs (Hair et al., 2022). When using Mode A (i.e., correlation weights), the bivariate correlation between each indicator and the construct determine the outer weights. In contrast, Mode B (i.e., regression weights) computes indicator weights by regressing each construct on its associated indicators.

7 To test the significant of $\omega$, the $95 \%$ percentile is often used for testing a two-sided hypothesis, in which the t-value must be greater than 1.96. However, if researchers are interested in testing a one-sided hypothesis, they can use the $90 \%$ percentile (Kock, 2015).

${ }^{8}$ To obtain further clarification of these equations for CoMe Models B-E in Table 2, researchers can contact the corresponding author.

${ }^{9}$ Hair et al. (2022) and Henseler and Fassot (2010) pointed out that when estimating a basic moderating effect, often researchers encounter the collinearity problem, especially when creating the interaction term from an exogenous construct and moderator. Thus, they typically standardize the indicators of the moderator before creating the interaction term. The standardization converts each variable 
to a mean of 0 and a standard deviation of 1 , which reduces collinearity introduced by indicator reuse. Furthermore, this standardization procedure helps facilitate the interpretation of the moderating effect. Based on these reasons, $\pm 1 S D$ is used in estimating the CoMe effect. However, researchers can easily adopt the \pm 2 SD or \pm 3 SD because using a wider interval of SD will help researchers show how the data are scattered.

${ }^{10}$ Before performing an MGA, the researcher should ensure that measurement invariance is achieved drawing on the measurement invariance of composite models (MICOM) procedure (see Henseler et al., 2016). By establishing MICOM, the researcher can be assured that the group differences in the model estimates do not result from distinct content and meanings of measurements used for the latent variables across groups. Notably, conducting an MGA requires partial measurement invariance, which is given when configural and compositional invariance hold. If partial measurement invariance is confirmed and the composites have equal mean values that vary across the groups, full measurement invariance is confirmed, which supports the pooled data analysis. In contrast, if partial measurement invariance is not present, this absence can deflate the power of statistical testing, influence the accuracy of estimators, and provide misleading results in further analyses.

${ }^{11}$ To test the significant difference in the mediated effect, the $95 \%$ percentile is often used for testing a two-sided hypothesis, in which the t-value must be greater than 1.96. For instance, the CoMe effect of $A$ on $B$ via $M$ will be different in groups 1 and 2. However, if researchers are interested in testing a one-sided hypothesis, they can use the $90 \%$ percentile. For instance, the CoMe effect of $A$ on $B$ via $M$ will be higher in group 1 than in group 2. Furthermore, if researchers are interested in examining the differences in the mediated effect of more than two groups, either Bonferroni correction or the Šidák procedure are the standard approach for controlling for the familywise error rate (Hair et al., 2018, p. 282).

12 The term "sugrophobia" indicates consumers' fear of being taken advantage of, duped, and exploited by salespeople, especially when they are less savvy or experienced regarding a purchase (Vohs et al., 2007). Consumers experience sugrophobia when they are concerned about whether the salespeople judge them as less savvy or less experienced about a purchase. Moreover, consumers who are highly sugrophobic may show signs of paranoia and distrust in uncertainty during the purchasing, thus the effect could weaken the decision-making process from price image to behavioral intention (Cheah et al., 2020; Babin et al., 2016).

${ }^{13}$ For the transformation of continuous data, we used the median split. Additionally, we have conducted a mean split, and the estimated CoMe results for Models A-C are similar to the median split (see Table 2).

14 The descriptions of the procedures employed to assess the CoMe index $(\omega)$ and CoMe effects for CoMe Model A, CoMe Model B, and CoMe Model C (using Excel) are available from the corresponding author (or see Online Supplement OS.1).

15 The interaction effect $(\mathrm{PI} \times$ SUGRO) was computed by applying the two-stage approach (Becker et al., 2018).
Bootstrapping based on 10,000 subsamples was used to test the significance of the parameters. The hypothesized effects were assessed by applying a one-tailed test for a tdistribution $(90 \% \mathrm{Cl})$.

16 The standard deviation of SUGRO is 1.0013 .

17 If a continuous moderator is used in determining the different level of the CoMe effect, Preacher et al. (2007) suggest to use the Johnson-Neyman's plot rather than the simple slope that we use for a categorical moderator. Often, it is interpreted by examining the middle line (black color) and the upper and lower bound values. The middle line represents the mediated effect, in which the top point of the middle line represents a low conditional mediated effect and the bottom point of the middle line represents a high conditional mediated effect. Looking at Figure 10 (Panel A), the mediated effect becomes weaker based on the conditional effect of SUGRO. If a categorical moderator is used in determining the CoMe effect, one can use the simple slope suggested by Preacher et al. (2017), in which the researcher can compare the direction and steepness (or gradient) of the CoMe effects for low and high values of the moderator (in our case this is Low Sugro and High Sugro) (see Figure 10, Panel B).

18 If the CoMe index $(\omega)$ is nonsignificant, researchers should stop assessing the CoMe effects for different levels of the moderator; rather, they should analyze moderation and mediation separately (see Figure 1). In our case, we continue with the empirical illustration by showing the steps on how to obtain the results of the CoMe effects for different levels of the moderator.

19 The standard deviation of SUGRO is 1.0011

20 When the analysis aims to examine differences in the strength (magnitude) of the mediated effects, the researcher could transform the data into categorical data for $\mathrm{CoMe}$ Model $C$ and perform an MGA test. For the details of the steps for doing this, researchers can refer to Section 4.2.

21 The standard deviation of SUGRO is 1.0012 .

${ }^{22}$ For further explanations on in-sample and out-of-sample predictions, please refer to Chin et al. (2020).

${ }^{23}$ Many empirical studies have demonstrated differences between men and women's behavior on their information processing and purchase decision-making, such as in social media (Pivec \& Maček, 2019), consumer purchase behavior (Lim et al., 2021), and in other m-commerce settings (Faqih \& Jaradat, 2015). Therefore, examining gender as the potential moderator variable for any mediated effect is warranted.

24 See Appendix E.

25 This analysis was carried out using SmartPLS 3.3.3 (Ringle et al., 2015).

${ }^{26}$ The path model was re-estimated using a bootstrapping routine based on 10,000 samples to test the significance of the parameters. Regarding the assessment of the CoMe effect, because we provide a one-sided hypothesis, we applied a one-tailed test $(90 \% \mathrm{Cl})$.

27 However, when the analysis aims to examine the rate of change (or slope) of the mediated relationship, researcher could refer to the steps in Section 4.1 to assess both the CoMe index $(\omega)$ and at different moderator levels for the CoMe effects. 


\section{References}

Aguinis, H., Edwards, J. R., \& Bradley, K. J. (2017). Improving our understanding of moderation and mediation in strategic management research. Organizational Research Methods, 20(4), 665685.

Aguinis, H., \& Gottfredson, R. K. (2010). Best-practice recommendations for estimating interaction effects using moderated multiple regression. Journal of Organizational Behavior, 31(6), 776786.

Aguirre-Urreta, M. I., \& Rönkkö, M. (2018). Statistical inference with PLSc using bootstrap confidence intervals. MIS Quarterly, 42(3), 1001-1020.

Ahadzadeh, A. S., Pahlevan Sharif, S., \& Sim Ong, F. (2018). Online health information seeking among women: The moderating role of health consciousness. Online Information Review, 42(1), 58-72.

Babin, B. J., Borges, A., \& James, K. (2016). The role of retail price image in a multi-country context: France and the USA. Journal of Business Research, 69(3), 1074-1081.

Bachl, M. (2017). Conditional process modeling (mediation analysis, moderated mediation analysis, moderation analysis, and mediated moderation analysis). The International Encyclopedia of Communication Research Methods, 1-26.

Baron, R. M., \& Kenny, D. A. (1986). The moderatormediator variable distinction in social psychological research: Conceptual, strategic, and statistical considerations. Journal of Personality and Social Psychology, 51(6), 11731182.

Becker, J.-M., \& Ismail, I. R. (2016). Accounting for sampling weights in PLS path modeling: Simulations and empirical examples. European Management Journal, 34(6), 606-617.

Becker, J.-M., Ringle, C. M., \& Sarstedt, M. (2018). Estimating moderating effects in PLS-SEM and PLSc-SEM: Interaction term generation*data treatment. Journal of Applied Structural Equation Modeling, 2(2), 1-21.

Becker, J.-M., Ringle, C. M., Sarstedt, M., \& Völckner, F. (2015). How collinearity affects mixture regression results. Marketing Letters, 26(4), 643659.

Borau, S., El Akremi, A., Elgaaied-Gambier, L., Hamdi-Kidar, L., \& Ranchoux, C. (2015). Analysing moderated mediation effects: Marketing applications. Recherche et Applications en Marketing (English Edition), 30(4), 88-128.
Calvo-Mora, A., Navarro-García, A., Rey-Moreno, M., \& Periañez-Cristobal, R. (2016). Excellence management practices, knowledge management and key business results in large organisations and SMEs: A multi-group analysis. European Management Journal, 34(6), 661-673.

Carrión, G. C., Nitzl, C., \& Roldán, J. L. (2017). Mediation analyses in partial least squares structural equation modeling: Guidelines and empirical examples. In H. Latan \& R. Noonan (Eds.), Partial Least Squares Path Modeling (pp. 173-195). Heidelberg: Springer.

Cheah, J.-H., Roldán, J. L., Ciavolino, E., Ting, H., \& Ramayah, T. (2020a). Sampling weight adjustments in partial least squares structural equation modeling: Guidelines and illustrations. Total Quality Management and Business Excellence. Forthcoming.

Cheah, J.-H., Sarstedt, M., Ringle, C. M., Ramayah, T., \& Ting, H. (2018). Convergent validity assessment of formatively measured constructs in PLS-SEM: On using single-item versus multiitem measures in redundancy analyses. International Journal of Contemporary Hospitality Management, 30(11), 3192-3210.

Cheah, J.-H., Waller, D., Thaichon, P., Ting, H., \& Lim, X.-J. (2020b). Price image and the sugrophobia effect on luxury retail purchase intention. Journal of Retailing and Consumer Services, 57, 102188.

Chin, W., Cheah, J.-H., Liu, Y., Ting, H., Lim, X.-J., \& Cham, T. H. (2020). Demystifying the role of causal-predictive modeling using partial least squares structural equation modeling in information systems research. Industrial Management and Data Systems, 120(12), 21612209.

Chin, W. W., \& Dibbern, J. (2010). A permutation based procedure for multi-group PLS analysis: Results of tests of differences on simulated data and a cross cultural analysis of the sourcing of information system services between Germany and the USA. In V. Esposito Vinzi, W. W. Chin, J. Henseler, \& H. Wang (Eds.), Handbook of Partial Least Squares: Concepts, Methods and Applications (Springer Handbooks of Computational Statistics Series, vol. II) (pp. 171193). Heidelberg, Dordrecht, London, New York: Springer.

Chin, W. W., Marcolin, B. L., \& Newsted, P. R. (2003). A partial least squares latent variable modeling approach for measuring interaction effects: Results from a Monte Carlo simulation study and an electronic-mail emotion/adoption study. Information Systems Research, 14(2), 189-217. 
Curran, P. J., \& Bauer, D. J. (2011). The disaggregation of within-person and betweenperson effects in longitudinal models of change. Annual Review of Psychology, 62(1), 583-619.

Danks, N. P., Sharma, P. N., \& Sarstedt, M. (2020). Model selection uncertainty and multimodel inference in partial least squares structural equation modeling (PLS-SEM). Journal of Business Research, 113, 13-24.

Dawson, J. F. (2014). Moderation in management research: What, why, when, and how. Journal of Business and Psychology, 29(1), 1-19.

DeCoster, J., Iselin, A., \& Gallucci, M. (2009). A conceptual and empirical examination of justifications for dichotomization. Psychological Methods, 14(4), 349-366.

Demming, C. L., Jahn, S., \& Boztug, Y. (2017). Conducting mediation analysis in marketing research. Marketing ZFP, 39(3), 76-98.

Dijkstra, T. K., \& Henseler, J. (2015a). Consistent and asymptotically normal PLS estimators for linear structural equations. Computational Statistics and Data Analysis, 81, 10-23.

Dijkstra, T. K., \& Henseler, J. (2015b). Consistent partial least squares path modeling. MIS Quarterly, 39(2), 297-316.

Edwards, J. R., \& Lambert, L. S. (2007). Methods for integrating moderation and mediation: A general analytical framework using moderated path analysis. Psychological Methods, 12(1), 1-22.

Faqih, K. M. S., \& Jaradat, M. R. (2015). Mobile healthcare adoption among patients in a developing country environment: Exploring the influence of age and gender differences. International Business Research, 8(9), 142.

Fassott, G., Henseler, J., \& Coelho, P. S. (2016). Testing moderating effects in PLS path models with composite variables. Industrial Management and Data Systems, 116(9), 1887-1900.

Fu, Y., Liu, X., Wang, Y., \& Chao, R.-F. (2018). How experiential consumption moderates the effects of souvenir authenticity on behavioral intention through perceived value. Tourism Management, 69, 356-367.

Gardner, R. G., Harris, T. B., Li, N., Kirkman, B. L., \& Mathieu, J. E. (2017). Understanding "it depends" in organizational research: A theory-based taxonomy, review, and future research agenda concerning interactive and quadratic relationships. Organizational Research Methods, 20(4), 610-638.

Gudergan, S. P., Ringle, C. M., Wende, S., \& Will, A. (2008). Confirmatory tetrad analysis in PLS path modeling. Journal of Business Research, 61(12), 1238-1249.
Hair, J. F., Hult, G. T. M., Ringle, C., \& Sarstedt, M. (2022). A primer on partial least squares structural equation modeling (PLS-SEM) ( $3^{\text {rd }}$ ed.). Sage Publications.

Hair, J. F., Risher, J. J., Sarstedt, M., \& Ringle, C. M. (2019). When to use and how to report the results of PLS-SEM. European Business Review, 31(1), 2-24.

Hair, J. F., \& Sarstedt, M. (2019). Factors versus composites: Guidelines for choosing the right structural equation modeling method. Project Management Journal, 50(6), 619-624.

Hair, J. F., Sarstedt, M., Ringle, C. M., \& Gudergan, S. P. (2018). Advanced issues in partial least squares structural equation modeling (PLS-SEM). Thousand Oaks, CA: SAGE.

Hair Jr., J. F. (2020). Next-generation prediction metrics for composite-based PLS-SEM. Industrial Management and Data Systems, 121(1), 5-11.

Hair Jr., J. F., Howard, M. C., \& Nitzl, C. (2020). Assessing measurement model quality in PLSSEM using confirmatory composite analysis. Journal of Business Research, 109, 101-110.

Hayes, A. F. (2015). An index and test of linear moderated mediation. Multivariate Behavioral Research, 50(1), 1-22.

Hayes, A. F. (2017). Introduction to mediation, moderation, and conditional process analysis: $A$ regression-based approach ( $2^{\text {nd }}$ ed.). Guilford Publications.

Hayes, A. F. (2018). Partial, conditional, and moderated mediation: Quantification, inference, and interpretation. Communication Monographs, 85(1), 4-40.

Hayes, A. F., \& Preacher, K. J. (2013). Conditional process modeling: Using structural equation modeling to examine contingent causal processes. In G. R. Hancock \& R. O. Mueller (Eds.), Structural equation modeling: A second course (pp. 219-266). IAP Information Age Publishing.

Hayes, A. F., \& Preacher, K. J. (2014). Statistical mediation analysis with a multicategorical independent variable. British Journal of Mathematical and Statistical Psychology, 67(3), 451-470.

Hayes, A. F., \& Rockwood, N. J. (2020). Conditional process analysis: Concepts, computation, and advances in the modeling of the contingencies of mechanisms. American Behavioral Scientist, 64(1), 19-54.

Hayes, A. F., \& Scharkow, M. (2013). The relative trustworthiness of inferential tests of the indirect effect in statistical mediation analysis: Does method really matter? Psychological Science, 24(10), 1918-1927. 
Henseler, J., \& Fassott, G. (2010). Testing moderating effects in PLS path models: An illustration of available procedures. In V. Esposito Vinzi, W. W. Chin, J. Henseler, \& H. Wang (Eds.), Handbook of partial least squares: Concepts, methods and applications (pp. 713-735). Berlin, Heidelberg: Springer Berlin Heidelberg.

Henseler, J., Ringle, C. M., \& Sarstedt, M. (2015). A new criterion for assessing discriminant validity in variance-based structural equation modeling. Journal of the Academy of Marketing Science, 43(1), 115-135.

Henseler, J., Ringle, C. M., \& Sarstedt, M. (2016). Testing measurement invariance of composites using partial least squares. International Marketing Review, 33(3), 405-431.

Henseler, J., Ringle, C. M., \& Sinkovics, R. R. (2009). The use of partial least squares path modeling in international marketing. In Advances in international marketing (pp. 277-319). Emerald Group Publishing Limited.

Hernandez-Ortega, B., Aldas-Manzano, J., Ruiz-Mafe, C., \& Sanz-Blas, S. (2017). Perceived value of advanced mobile messaging services: A crosscultural comparison of Greek and Spanish users. Information Technology and People, 30(2), 324355.

Holland, S. J., Shore, D. B., \& Cortina, J. M. (2017). Review and recommendations for integrating mediation and moderation. Organizational Research Methods, 20(4), 686-720.

Hult, G. T. M., Hair, J. F., Proksch, D., Sarstedt, M., Pinkwart, A., \& Ringle, C. M. (2018). Addressing endogeneity in international marketing applications of partial least squares structural equation modeling. Journal of International Marketing, 26(3), 1-21.

Hwang, H., Desarbo, W. S., \& Takane, Y. (2007). Fuzzy clusterwise generalized structured component analysis. Psychometrika, 72(2), 181198.

Hwang, H., Ho, M.-H. R., \& Lee, J. (2010). Generalized structured component analysis with latent interactions. Psychometrika, 75(2), 228242.

Hwang, H., Sarstedt, M., Cheah, J. H., \& Ringle, C. M. (2020). A concept analysis of methodological research on composite-based structural equation modeling: Bridging PLSPM and GSCA. Behaviormetrika, 47(1), 219-241.

Karazsia, B. T., van Dulmen, M. H., Wong, K., \& Crowther, J. H. (2013). Thinking metatheoretically about the role of internalization in the development of body dissatisfaction and body change behaviors. Body Image, 10(4), 433-441.
Keil, M., Tan, B. C. Y., Wei, K.-K., Saarinen, T., Tuunainen, V., \& Wassenaar, A. (2000). A crosscultural study on escalation of commitment behavior in software projects. MIS Quarterly, 24(2), 299-325.

Khan, G. F., Sarstedt, M., Shiau, W.-L., Hair, J. F., Ringle, C. M., \& Fritze, M. P. (2019). Methodological research on partial least squares structural equation modeling (PLS-SEM): An analysis based on social network approaches. Internet Research, 29(3), 407-429.

Klesel, M., Schuberth, F., Henseler, J., \& Niehaves, B. (2019). A test for multigroup comparison using partial least squares path modeling. Internet Research, 29(3), 464-477.

Klesel, M., Schuberth, F., Niehaves, B., \& Henseler, J. (2021). Multigroup analysis in information systems research using PLS-PM: A systematic investigation of approaches. The DATA BASE for Advances in Information Systems, forthcoming.

Koay, K. Y. (2018). Workplace ostracism and cyberloafing: A moderated-mediation model. Internet Research, 28(4), 1122-1141.

Kock, N. (2015). One-tailed or two-tailed $P$ values in PLS-SEM?. International Journal of $e$ Collaboration (IJeC), 11(2), 1-7.

Kock, N., \& Hadaya, P. (2018). Minimum sample size estimation in PLS-SEM: The inverse square root and gamma-exponential methods. Information Systems Journal, 28(1), 227-261.

Lance, C. E. (1988). Residual centering, exploratory and confirmatory moderator analysis, and decomposition of effects in path models containing interactions. Applied Psychological Measurement, 12(2), 163-175.

Leal-Rodríguez, A. L., Ariza-Montes, J. A., Roldán, J. L., \& Leal-Millán, A. G. (2014). Absorptive capacity, innovation and cultural barriers: A conditional mediation model. Journal of Business Research, 67(5), 763-768.

Liengaard, B. D., Sharma, P. N., Hult, G. T. M., Jensen, M. B., Sarstedt, M., Hair, J. F., \& Ringle, C. M. (2021). Prediction: Coveted, yet forsaken? Introducing a cross-validated predictive ability test in partial least squares path modeling. Decision Sciences, 52(2), 362-392.

Lim, X. J., Cheah, J. H., Ng, S. I., Basha, N. K., \& Liu, Y. (2021). Are men from Mars, women from Venus? Examining gender differences towards continuous use intention of branded apps. Journal of Retailing and Consumer Services, 60, 102422.

Limaj, E., \& Bernroider, E. W. N. (2019). The roles of absorptive capacity and cultural balance for exploratory and exploitative innovation in SMEs. Journal of Business Research, 94, 137-153. 
Marcoulides, G. A., \& Chin, W. W. (2013). You write, but others read: Common methodological misunderstandings in PLS and related methods. In Springer Proceedings in Mathematics and Statistics (pp. 31-64). Springer.

Mason, C. H., \& Perreault Jr, W. D. (1991). Collinearity, power, and interpretation of multiple regression analysis. Journal of Marketing Research, 28(3), 268-280.

Mathieu, J. E., DeShon, R. P., \& Bergh, D. D. (2008). Mediational inferences in organizational research: Then, now, and beyond. Organizational Research Methods, 11(2), 203-223.

Morgan-Lopez, A. A., \& MacKinnon, D. P. (2006). Demonstration and evaluation of a method for assessing mediated moderation. Behavior Research Methods, 38(1), 77-87.

Muller, D., Judd, C. M., \& Yzerbyt, V. Y. (2005). When moderation is mediated and mediation is moderated. Journal of Personality and Social Psychology, 89(6), 852-863.

Nitzl, C., Roldán, J. L., \& Cepeda Carrión, G. (2016). Mediation analysis in partial least squares path modeling: Helping researchers discuss more sophisticated models. Industrial Management and Data Systems, 116(9), 1849-1864.

Park, H., Yoo, J.-Y., Moon, S.-H., Yoo, H.-S., Lee, H.S., Kwon, T.-H., \& Hahn, H. (2019). Effect of technology and market dynamism on the business performances of SMEs by supporting services. Science, Technology and Society, 24(1), 144-160.

Petter, S. (2018). 'Haters gonna hate': PLS and information systems research. The DATABASE for Advances in Information Systems, 49(2), 1013.

Pirlott, A. G., \& MacKinnon, D. P. (2016). Design approaches to experimental mediation. Journal of Experimental Social Psychology, 66, 29-38.

Pivec, M., \& Maček, A. (2019). Employment background influence on social media usage in the field of European project management and communication. Journal of Business Research, 94, 280-289.

Preacher, K. J., \& Hayes, A. F. (2008). Asymptotic and resampling strategies for assessing and comparing indirect effects in multiple mediator models. Behavior Research Methods, 40(3), 879891.

Preacher, K. J., Rucker, D. D., \& Hayes, A. F. (2007). Addressing moderated mediation hypotheses: Theory, methods, and prescriptions. Multivariate Behavioral Research, 42(1), 185-227.

$\mathrm{R}$ Core Team (2021). R: A language and environment for statistical computing. Vienna, Austria: $\mathrm{R}$ Foundation for Statistical Computing, available at https:. Retrieved from www.R-project.org.
Ratzmann, M., Gudergan, S., \& Bouncken, R. (2016). Capturing heterogeneity and PLS-SEM prediction ability: Alliance governance and innovation. Journal of Business Research, 69, 4593-4603.

Rezaei, S., \& Valaei, N. (2017). Branding in a multichannel retail environment: Online stores vs app stores and the effect of product type. Information Technology and People, 30(4), 853886.

Ringle, C. M., Wende, S., \& Becker, J.-M. (2015). SmartPLS 3. Bönningstedt: SmartPLS. Retrieved from http://www.smartpls.com.

Roemer, E. (2016). A tutorial on the use of PLS path modeling in longitudinal studies. Industrial Management and Data Systems, 116(9), 19011921.

Ryu, E., \& Cheong, J. (2017). Comparing indirect effects in different groups in single-group and multi-group structural equation models. Frontiers in Psychology, 8, 747.

Sarstedt, M., Hair Jr., J. F., Nitzl, C., Ringle, C. M., \& Howard, M. C. (2020a). Beyond a tandem analysis of SEM and PROCESS: Use of PLSSEM for mediation analyses! International Journal of Market Research, 62(3), 288-299.

Sarstedt, M., Henseler, J., \& Ringle, C. M. (2011). Multigroup analysis in partial least squares (PLS) path modeling: Alternative methods and empirical results. In Advances in international marketing (pp. 195-218). Emerald Group Publishing Limited.

Sarstedt, M., Ringle, C. M., Cheah, J.-H., Ting, H., Moisescu, O. I., \& Radomir, L. (2020b). Structural model robustness checks in PLS-SEM. Tourism Economics, 26(4), 531-554.

Sarstedt, M., Ringle, C., \& Gudergan, S. (2016). Guidelines for treating unobserved heterogeneity in tourism research: A comment on Marques and Reis (2015). Annals of Tourism Research, 57, 279-284.

Sarstedt, M., Ringle, C. M., \& Hair, J. F. (2017). Treating unobserved heterogeneity in PLS-SEM: A multi-method approach. In $\mathrm{H}$. Latan \& $\mathrm{R}$. Noonan (Eds.), Partial least squares path modeling (pp. 197-217). Springer, Cham.

Sharma, P. N., Sarstedt, M., Shmueli, G., Kim, K. H., \& Thiele, K. O. (2019). PLS-based model selection: The role of alternative explanations in information systems research. Journal of the Association for Information Systems, 20(4), 346397.

Sharma, P. N., Sarstedt, M., Shmueli, G., Kim, K. H., \& Thiele, K. O. (2019). PLS-based model selection: The role of alternative explanations in information systems research. Journal of the Association of Information Systems, 20(4), 346397. 
Sharma, P. N., Shmueli, G., Sarstedt, M., Danks, N., \& Ray, S. (2021). Prediction-oriented model selection in partial least squares path modeling. Decision Sciences, 52(3), 567-607.

Shmueli, G., Ray, S., Velasquez Estrada, J. M., \& Chatla, S. B. (2016). The elephant in the room: Evaluating the predictive performance of PLS models. Journal of Business Research, 69(10), 4552-4564.

Shmueli, G., Sarstedt, M., Hair, J. F., Cheah, J.-H., Ting, H., Vaithilingam, S., \& Ringle, C. M. (2019). Predictive model assessment in PLS-SEM: Guidelines for using PLSpredict. European Journal of Marketing, 53(11), 2322-2347.

Shrout, P. E., \& Bolger, N. (2002). Mediation in experimental and nonexperimental studies: New procedures and recommendations. Psychological Methods, 7(4), 422-445.

Spector, P. E. (2019). Do not cross me: Optimizing the use of cross-sectional designs. Journal of Business and Psychology, 34(2), 125-137.

Spiller, S. A., Fitzsimons, G. J., Lynch Jr., J. G., \& McClelland, G. H. (2013). Spotlights, floodlights, and the magic number zero: Simple effects tests in moderated regression. Journal of Marketing Research, 50(2), 277-288.

Stone-Romero, E. F., \& Rosopa, P. J. (2011). Experimental tests of mediation models: Prospects, problems, and some solutions. Organizational Research Methods, 14(4), 631646.

Streukens, S., \& Leroi-Werelds, S. (2016a). Bootstrapping and PLS-SEM: A step-by-step guide to get more out of your bootstrap results. European Management Journal, 34(6), 618-632.

Streukens, S., \& Leroi-Werelds, S. (2016b). PLS FACSEM: An illustrated step-by-step guideline to obtain a unique insight in factorial data. Industrial Management and Data Systems, 116(9), 19221945.

Stride, C. B., Gardner, S., Catley, N., \& Thomas, F. (2015). Mplus code for mediation, moderation, and moderated mediation models. Retrieved from http://www.offbeat.group.shef.ac.uk/FIO/mplusm edmod.htm

Tenenhaus, A., \& Tenenhaus, M. (2011). Regularized generalized canonical correlation analysis. Psychometrika, 76(2), 257-284.

Vandenberg, R. J., \& Lance, C. E. (2000). A review and synthesis of the measurement invariance literature: Suggestions, practices, and recommendations for organizational research. Organizational Research Methods, 3(1), 4-70.
Vohs, K. D., Baumeister, R. F., \& Chin, J. (2007). Feeling duped: Emotional, motivational, and cognitive aspects of being exploited by others. Review of General Psychology, 11(2), 127-141.

Wang, L., \& Preacher, K. J. (2015). Moderated mediation analysis using Bayesian methods. Structural Equation Modeling: A Multidisciplinary Journal, 22(2), 249-263.

Welch, B. L. (1947). The generalization of "Student's" problem when several different population variances are involved. Biometrika, 34(1/2), 2835.

Wooldridge, J. M. (2015). Introductory econometrics: A modern approach. Cengage learning, USA.

Willems, K., Brengman, M., \& Van Kerrebroeck, H. (2019). The impact of representation media on customer engagement in tourism marketing among millennials. European Journal of Marketing, 53(9), 1988-2017.

Zhao, X., Lynch Jr, J. G., \& Chen, Q. (2010). Reconsidering Baron and Kenny: Myths and truths about mediation analysis. Journal of Consumer Research, 37(2), 197-206.

\section{About the Authors}

Jun-Hwa Cheah (Jacky) is a Senior Lecturer in School of Business and Economics, Universiti Putra Malaysia (UPM) as well as an external member of relationship marketing for impact cluster at Griffith University. His areas of interest include consumer behavior, quantitative research, and methodological issue. His publications appear in journals such as European Journal of Marketing, Journal of Retailing and Consumer Services, Marketing Intelligence and Planning, Asia Pacific Journal Marketing and Logistics, Technological Forecasting and Social Change, Total Quality Management and Business Excellent, Management Decision, Internet Research, Information Systems Management, Industrial Management and Data Systems, Tourism Management, Tourism Economics, International Journal of Contemporary Hospitality Management, and other. $\mathrm{He}$ has also received several research awards, regularly coaches researchers and practitioners, and is a frequently invited speaker.

Christian Nitzl is Senior Researcher at the Center for Intelligence and Security Studies (CISS). His interests include the development and application of path models, the application of artificial intelligence/big data in intelligence and security related areas, and human decision behavior. Christian Nitzl has published in Acta Astronautica, Business Research, Financial Accountability \& Management, Hospital Pharmacy, Industrial Management \& Data Systems, Journal of Accounting Literature, Journal of Accounting \& Organizational Change, Journal of Banking and 
Finance, Journal of Business Research, Journal of Health Organization and Management, Journal of Management Control, Journal of Public and Nonprofit Management, Public Management Review, and Die Betriebswirtschaft, among others. So far, he has worked several times as a reviewer for top-class professional journals. Christian Nitzl has received several awards. In addition, he regularly coaches researchers and practitioners and is a frequently invited speaker.

José L. Roldán, PhD, is a Professor of Management in the Department of Business Administration and Marketing, Universidad de Sevilla, Spain. His research interests include technology acceptance models, knowledge management, organizational culture, organizational agility, and partial least squares (PLS). His recent contributions have been published in Decision Sciences, Internet Research, Journal of Travel Research, European Journal of Operational Research, International Journal of Project Management, British Journal of Management, Journal of Business Research, European Journal of Information Systems, Computers in Human Behavior, and Industrial Marketing Management, among others. $\mathrm{He}$ is currently on the editorial board of The Data Base for Advances in Information Systems. He has also served as Guest Editor of the European Journal of Information Systems, Journal of Business Research, European Management Journal, and International Journal of Sports Marketing and Sponsorship. In addition, Dr. Roldan has been conference co-chair of the 2nd International Symposium on Partial Least Squares Path Modeling-The Conference for PLS Users (June 16-19, Seville, Spain) and the 9th International Conference on PLS and Related Methods (PLS'17) (June 17-19, 2017, Macau, China).

Gabriel Cepeda-Carrion is a Professor of Management in the Department of Management and Marketing, at Universidad de Sevilla, Spain. His research interests include dynamic capabilities, knowledge management, leadership, sport management, and partial least squares structural equation modeling (PLS-SEM). His recent contributions have been published in Journal of Knowledge Management, Internet Research, Industrial Management \& Data Systems, European Management Journal, Management Decision, British Journal of Management, Journal of Business Research, Journal of Organizational Change Management, Journal of Services Marketing, and Total Quality Management \& Business Excellence, among others. He is currently on the editorial board of the International Journal of Sports Marketing and Sponsorship. He has also served as Guest Editor of the Journal of Business Research, European Management Journal, and International Journal of Sports Marketing and Sponsorship. In addition, Dr. Cepeda has been conference co-chair of the 2nd International Symposium on Partial Least Squares Path Modeling-The Conference for PLS Users (June 16-19, Seville, Spain) and the 9th International Conference on PLS and Related Methods (PLS'17) (June 17-19, 2017, Macau, China).

Siegfried P. Gudergan is Professor of Strategy within the College of Business, Law \& Governance at James Cook University Australia. He has earned a PhD from the former Australian Graduate School of Management that was awarded by both the University of Sydney and UNSW. His research spans the areas of business strategy and organizational innovation (e.g., dynamic capabilities), managerial decision making (e.g., heuristics), customer experiences (e.g., customer engagement), and partial least squares structural equation modelling (PLS-SEM). His publications have appeared in, for example, the Journal of the Academy of Marketing Science, International Journal of Research in Marketing, Long Range Planning, Journal of International Management, Journal of Business Research, Tourism Management, and Journal of Product Innovation Management, among others. 


\section{Appendix A. Assessment of Confirmatory Tetrad Analysis}

\begin{tabular}{|l|l|c|c|c|c|c|c|}
\hline & & & & & \multicolumn{2}{|c|}{ Cl adj $^{*}$} & \\
\hline Construct & $\begin{array}{l}\text { Model Implied Non-Redundant } \\
\text { Vanishing Tetrad }\end{array}$ & $\begin{array}{c}\text { Tetrad } \\
\text { Value }\end{array}$ & $\begin{array}{c}\text { Standard } \\
\text { Error }\end{array}$ & t-value & $\begin{array}{c}\text { Lower } \\
\text { Bound }\end{array}$ & $\begin{array}{c}\text { Upper } \\
\text { Bound }\end{array}$ & $\begin{array}{c}\text { Supported } \\
\text { Reflective }\end{array}$ \\
\hline $\begin{array}{l}\text { Behavioral } \\
\text { Intention }\end{array}$ & 1: Attitude2, Attitude3, BI1, BI2 & 0.317 & 0.055 & 5.736 & 0.210 & 0.428 & No \\
\hline Price Image & 2: Attitude2, Attitude3, BI2, BI1 & 0.293 & 0.055 & 5.300 & 0.186 & 0.402 & \\
\hline 1: PI1, PI2, PI3, PI4 & 0.103 & 0.066 & 1.561 & -0.005 & 0.216 & Yes \\
\hline $\begin{array}{l}\text { Perceived } \\
\text { Value }\end{array}$ & 2: PI1, PI2, PI4, PI3 & -0.191 & 0.125 & 1.528 & -0.411 & 0.040 & \\
\hline & 1: PV1, PV2, PV3, Attitude1 & -0.016 & 0.059 & 0.270 & -0.129 & 0.102 & Yes \\
\hline Sugrophobia & $\begin{array}{l}\text { 2: PV1, PV2, Attitude 2, PV3 } \\
\text { Sugro3 }\end{array}$ & -0.082 & 0.063 & 1.288 & -0.201 & 0.048 & \\
\hline & $\begin{array}{l}\text { 2: Inertia1, Sugro1, Sugro3, } \\
\text { Sugro2 }\end{array}$ & 0.082 & 0.093 & 0.376 & -0.107 & 0.257 & \\
\hline
\end{tabular}

Note: (i) * means that $\mathrm{Cl}$ adj $=90 \%$ bias corrected and Bonferroni-adjusted bootstrap confidence interval and (ii) Since confirmatory tetrad analysis (CTA) does not function for latent variables with less than four items - in our case behavioral intention, perceived value, and sugrophobia - therefore, this study borrows other somewhat related items from attitude and inertia constructs with the highest correlation and highest loading (see Gudergan et al., 2008). 


\section{Appendix B(i). Step-by-Step Procedure for Conditional Mediation Analysis of CoMe Model A Using a Continuous Moderator}

To obtain the index of " $p_{2} \cdot p_{5}$ ", this result can be directly obtained from any type of PLS-SEM software (e.g., SmartPLS). Specifically, the researcher needs to estimate the path coefficients using the bootstrapping output of the specific mediated effect. After the convergence of the bootstrapping result, the researcher needs to examine the significance of "PI*SUGRO $\rightarrow P V \rightarrow B l^{*}$ ' from the specific mediated effect output (see Figure B1, Panel A and Panel B).

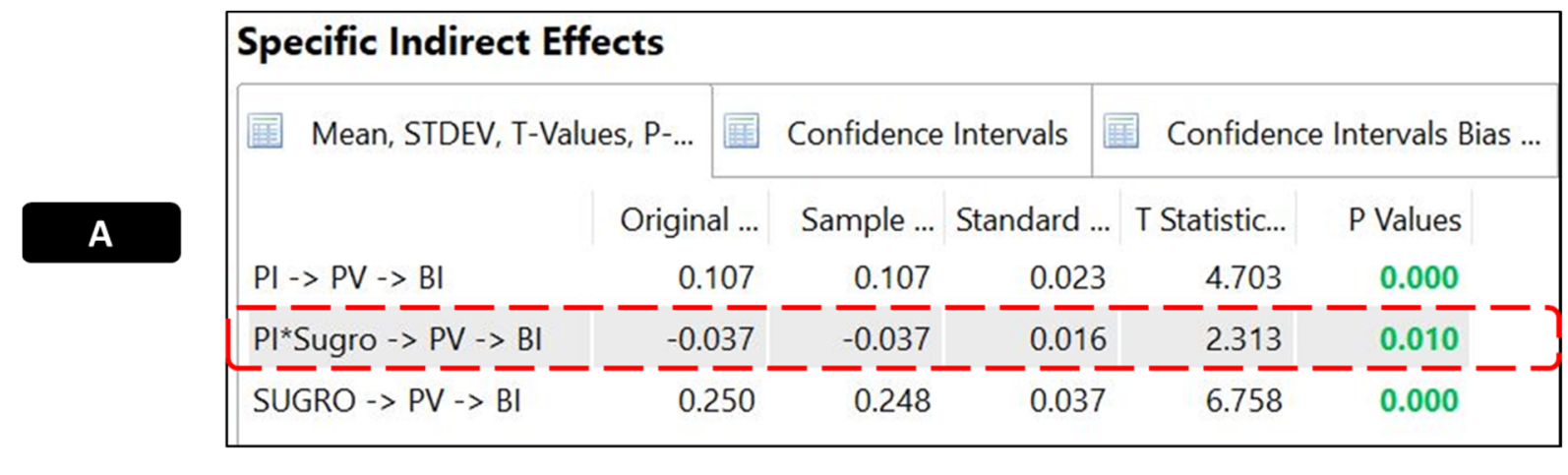

\begin{tabular}{|c|c|c|c|c|c|c|}
\hline & \multicolumn{6}{|c|}{ Specific Indirect Effects } \\
\hline & Mean, STDEV, T-V & es, P-... 贯 & Confidence & rvals & 囯 & Confidence Intervals Bias ... \\
\hline \multirow[t]{4}{*}{ B } & & Original ... & Sample ... & $5.0 \%$ & & $95.0 \%$ \\
\hline & $\mathrm{PI}->\mathrm{PV}->\mathrm{BI}$ & 0.107 & 0.107 & 0.070 & & 0.145 \\
\hline & $\mathrm{Pl}^{*}$ Sugro $\rightarrow \mathrm{PV} \rightarrow \mathrm{BI}$ & -0.037 & $-\overline{-0.037}$ & $-0 . \overline{065}$ & & -0.010 \\
\hline & SUGRO $->\mathrm{PV}>\overline{\mathrm{BI}}$ & $\overline{0.250}$ & $0 . \overline{248}$ & 0.188 & & $\overline{0.310}$ \\
\hline
\end{tabular}

Figure B1. (Panel A) The p-value Result for the Index of " $p_{2} \cdot p_{5}$ " and (Panel B) the Percentile-Corrected Result for the Index of " $p_{2} \cdot p_{5}$ "

Next, we examine the mediated effect at different levels of the moderator. To formally test this for CoMe Model A, we initially assess the standard deviation of the moderator (SUGRO), as it will denote that the moderator is one standard deviation below and one standard deviation above the mean. The standard deviation for SUGRO is 1.0013. This standard deviation value can be obtained, for example, via the SPSS software or the use of an Excel spreadsheet. Subsequently, using a spreadsheet application (i.e., MS Excel), we suggest carrying out the following steps:

1) Take the 10,000 path coefficients from all direct effects created by the bootstrap sampling and copy and paste them into a spreadsheet (see Figure B2). 


\begin{tabular}{|c|c|c|c|c|c|c|}
\hline$\angle$ & A & B & C & D & $\mathrm{E}$ & $\mathrm{F}$ \\
\hline \multicolumn{7}{|c|}{1} \\
\hline \multicolumn{7}{|l|}{2} \\
\hline \multicolumn{7}{|l|}{3} \\
\hline \multicolumn{7}{|l|}{4} \\
\hline \multicolumn{7}{|l|}{5} \\
\hline \multicolumn{7}{|l|}{6} \\
\hline 7 & & p1 & p2 & p3 & $\mathrm{p} 4$ & p5 \\
\hline 8 & & \multicolumn{2}{|c|}{$\mathrm{PI}->\mathrm{PV} P V \rightarrow \mathrm{BI}$} & $\mathrm{PI} \rightarrow \mathrm{BI}$ & SUGRO $\rightarrow$ PV & PI*SUGRO -> PV \\
\hline 9 & Sample 0 & 0.179 & 0.533 & 0.289 & 0.547 & -0.076 \\
\hline 10 & Sample 1 & 0.249 & 0.503 & 0.295 & 0.472 & -0.086 \\
\hline 11 & Sample 2 & 0.229 & 0.469 & 0.333 & 0.512 & -0.056 \\
\hline 12 & Sample 3 & 0.221 & 0.513 & 0.263 & 0.469 & -0.037 \\
\hline 13 & Sample 4 & 0.192 & 0.513 & 0.289 & 0.418 & -0.151 \\
\hline 14 & Sample 5 & 0.216 & 0.397 & 0.370 & 0.484 & -0.081 \\
\hline
\end{tabular}

Figure B2. Step 1 Illustrative Example for Use of Continuous Moderator (Model A)

2) Based on columns $B$ to $F$ in Figure $B 2$, create a new column for the CoMe effects at low, medium, and high levels of the moderator using the step-by-step illustrated equations suggested in Figure B3.

Create each of these columns using the illustrated equations 6 to 8 in Table 1 (see main body of paper).

Drawing on these equations enables obtaining the subsample results of the CoMe effect at low moderator level, CoMe effect at medium moderator level, and CoMe effect at high moderator level.

\begin{tabular}{|c|c|c|c|c|c|c|c|c|c|c|}
\hline$\Delta$ & 1 & J & $\mathrm{K}$ & L & M & $\mathrm{N}$ & 0 & $\mathrm{P}$ & Q & $\mathrm{R}$ \\
\hline 1 & Original (0) & & & & & & & & & \\
\hline 2 & Lower Percentile (5\%) & & & & & & & & & \\
\hline 3 & Upper Percentile (95\%) & & & & & & & & & \\
\hline 4 & Standard Error & & & & & & & & & \\
\hline 5 & t-value & & & & & & & & & \\
\hline 6 & & rா-ーーーーー & $--\infty$ & ーーーーーーーー & -ーーーーーーーー & -ーーーーー & ローーーーーーーーー & ーーーーーーーーーーーー & -ーtーーーーーー & ーーーーーーーーーーーーー \\
\hline 7 & & & & & & & & Low CoMe & Medium CoMe & High CoMe \\
\hline 8 & Formula & $p 5 *(0-1.0013)$ & $p 5 *(0)$ & $p 5 *(0+1.0013)$ & $p 1+p 5^{*}(0-1.0013)$ & $p 1+p 5 *(0)$ & $\mathrm{p} 1+\mathrm{p} 5 *(0+1.0013)$ & $\left(p 1+p 5^{*}(0-1.0013)\right)^{*} p 2$ & $\left(p 1+p 5^{*}(0)\right)^{*} p 2$ & $(p 1+p 5 *(0+1.0013))^{*} p 2$ \\
\hline 9 & & 0.076 & 0.000 & -0.076 & 0.255 & 0.179 & 0.103 & 0.136 & 0.095 & 0.055 \\
\hline 10 & & 0.086 & 0.000 & -0.086 & 0.335 & 0.249 & 0.163 & 0.169 & 0.125 & 0.082 \\
\hline 11 & & 0.056 & 0.000 & -0.056 & 0.285 & 0.229 & 0.173 & 0.134 & 0.107 & 0.081 \\
\hline 12 & & 0.037 & 0.000 & -0.037 & 0.258 & 0.221 & 0.184 & 0.132 & 0.113 & 0.094 \\
\hline 13 & & L__ 0.151 & 0.000 & $-0.0 .151=$ & 0.343 & 0.192 & - 0.041 & 0.176 & _o. 0.098 & _o. 0.021 \\
\hline
\end{tabular}

Figure B3. Step 2 Illustrative Example for Use of a Continuous Moderator (CoMe Model A)

3) Calculate the original values $(\mathrm{O})$ as provided by the PLS-SEM path coefficients for each subsample (see Figure B4). The results show that the path coefficient effects of the CoMe effects are weaker when the SUGRO effect is one standard deviation above the mean. 
Calculate the original values $(0)$ provided by PLS for path coefficients.

The details are: $=$ AVERAGE(P9:P10008), =AVERAGE(Q9:Q10008), and

$=$ AVERAGE(R9:R10008)

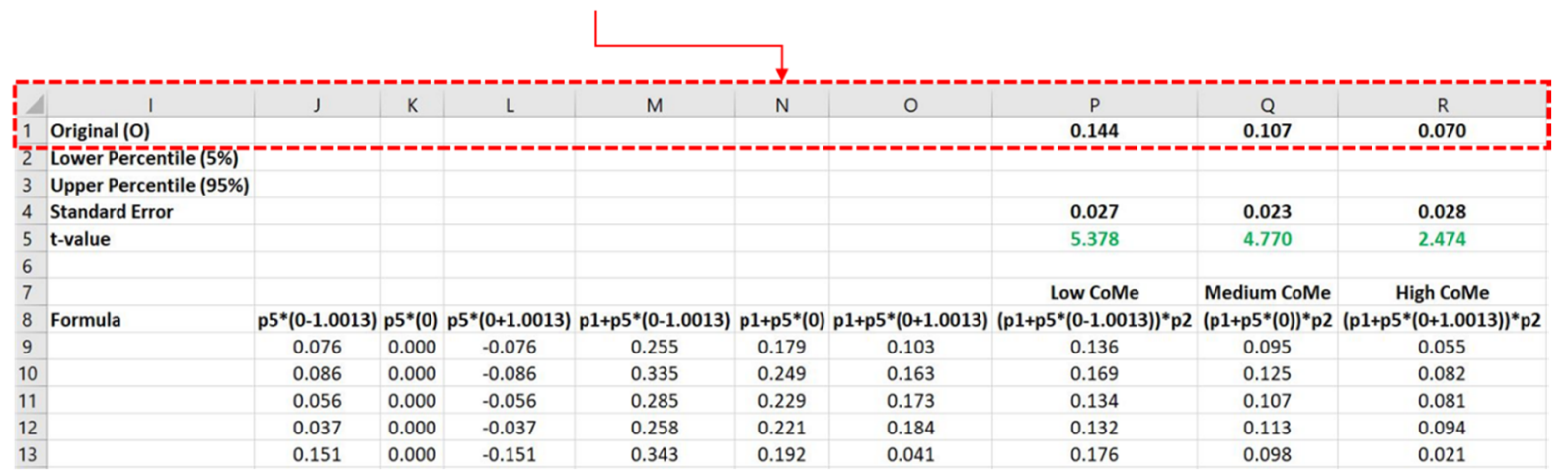

Figure B4. Step 3 Illustrative Example of for Use of Continuous Moderator (CoMe Model A)

4) Estimate the percentile bootstrap confidence interval $(\mathrm{Cl})$ using the function PERCENTILE (range, $\mathrm{k}$ ), where $\mathrm{k}$ is the percentile value between 0 and 1 (see Figure B5). If the $\mathrm{Cl}$ for the product "does not include a 0 value, this result means that the mediated effects at different levels of the moderator are significantly different from $0 . " 12$ In our example, the CoMe effects at low, medium, and high levels of the moderator are significant because both extremes of the $\mathrm{Cl}$ are positive. Therefore, we find some evidence to support the hypothesis of CoMe Model A; that is, the mediated effect of the consumer's PI on BI via PV reduces as SUGRO increases.

Calculate both the lower percentile (5\%) and the upper percentile (95\%).

- The details of the lower percentile for CoMe effect at low moderator level, CoMe effect at medium moderator level, and CoMe effect at high moderator level are: =PERCENTILE(P9:P10008, 0.05), =PERCENTILE(Q9:Q10008, 0.05), and =PERCENTILE(R9:R10008, 0.05)

- The details of the upper percentile for CoMe effect at low moderator level, CoMe effect at medium moderator level, and CoMe effect at high moderator level are: =PERCENTILE(P9:P10008, 0.95), =PERCENTILE(Q9:Q10008, 0.95), and =PERCENTILE(R9:R10008, 0.95)

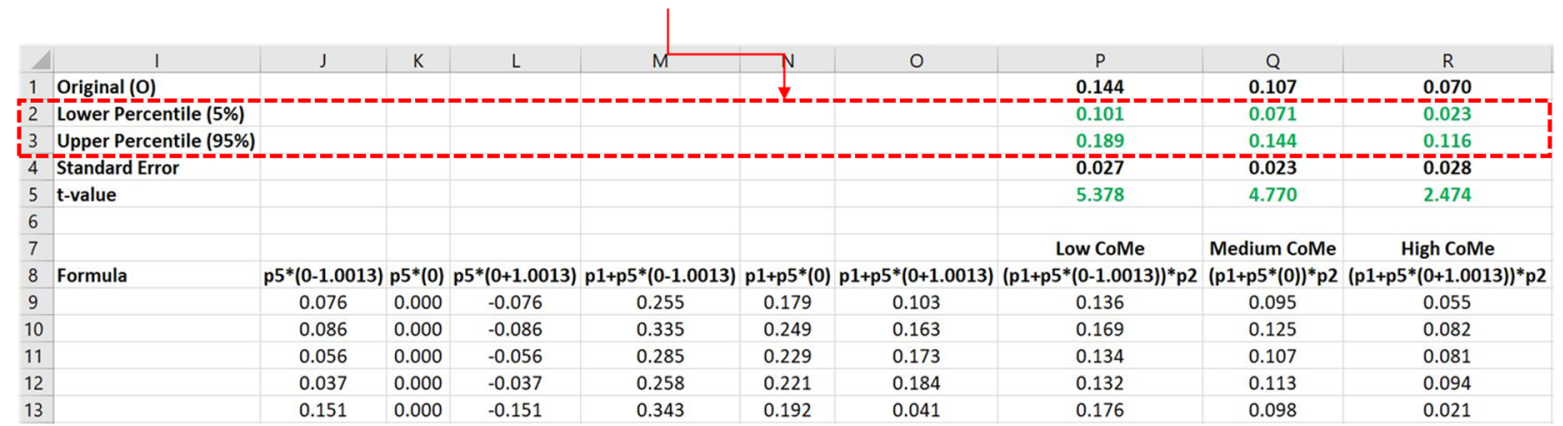

Figure B5. Step 4 Illustrative Example for Use of Continuous Moderator (CoMe Model A)

\footnotetext{
${ }^{1}$ Researchers could also in addition produce the t-value by first calculating the standard error (=STDEV(number 1 , number 2 , ...)). Next, use the original values $(\mathrm{O})$ divided by the standard error to obtain the t-value.

2 If researchers are interested in creating a graphical plot of the mediated effects at different levels of the moderator, they need to continue generating the standard error using the following formula: =STDEV(P9:P10008), =STDEV(Q9:Q10008), and $=S T D E V(R 9: R 10008)$ (see the result in Figure B5).
} 
5) Finally, the researcher can create a graphical plot of the mediated effects at different levels of the moderator by using the proposed Excel template (see https://github.com/cortrudolph/Conditional Indirect Effects or http://www.md2c.nl/). Once the researcher has inserted this result (see Figure B6), he or she can easily produce the graphical plot (see Figure 10 in the main body of this paper).

2) Second, insert the standard deviation value of the moderator (i.e. $-1.0013,0.0000,1.0013$ ). Subsequently,

1) First, insert the moderator name in the blue boxes (i.e., SUGRO) insert the values of both the original and standard error of the CoMe effect at low moderator level, CoMe effect at medium moderator level, and CoMe effect at high moderator level (see Figure C5 for these values)

A

\section{Graph the Conditional Mediated Effect}

Directions: Enter corresponding output information in the blue boxes:

Note 1: Calculated values appear in green boxes.

Note 2: Do not modify grey boxes.

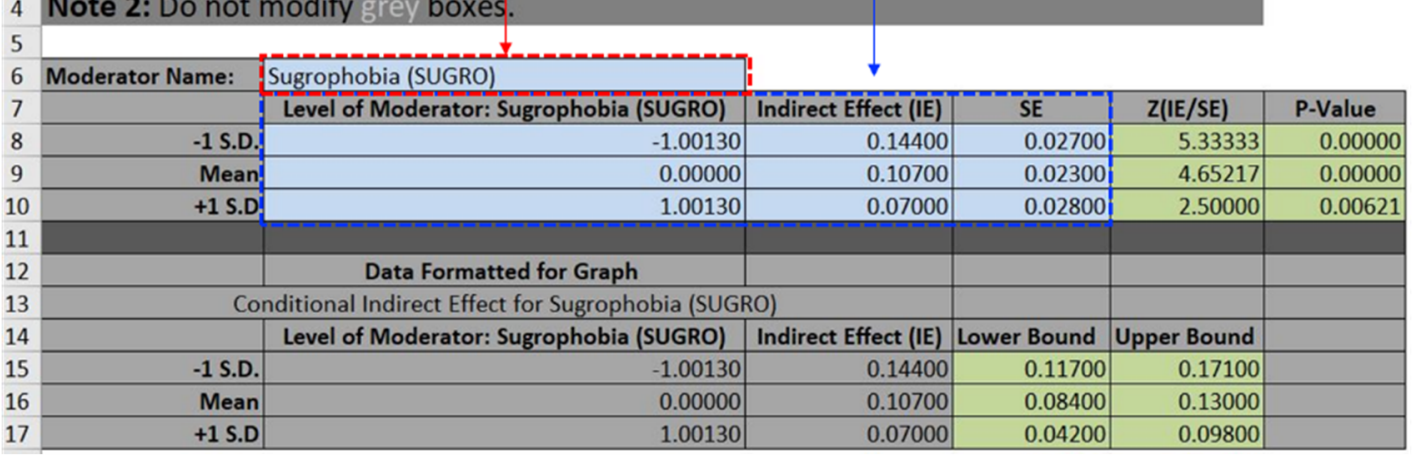

Figure B6. Step 5 Illustrative Example for Use of Continuous Moderator (CoMe Model A). 


\section{Appendix B(ii). Step-by-Step Procedure for Conditional Mediation Analysis of CoMe Model A When Moderator Is Converted from Continuous to Categorical}

To obtain the index of " $p_{2} \cdot p_{5}$ ", this result can be directly obtained from any type of PLS-SEM software even when continuous data has been transformed to categorical data via median split. Specifically, the researcher needs to estimate the path coefficients using the bootstrapping output of the specific mediated effect. After the convergence of the bootstrapping result, the researcher needs to examine the significance of "PI'SUGRO $->P V->B l$ " from the specific mediated effect output (see Figure B7, Panel A and Panel B).

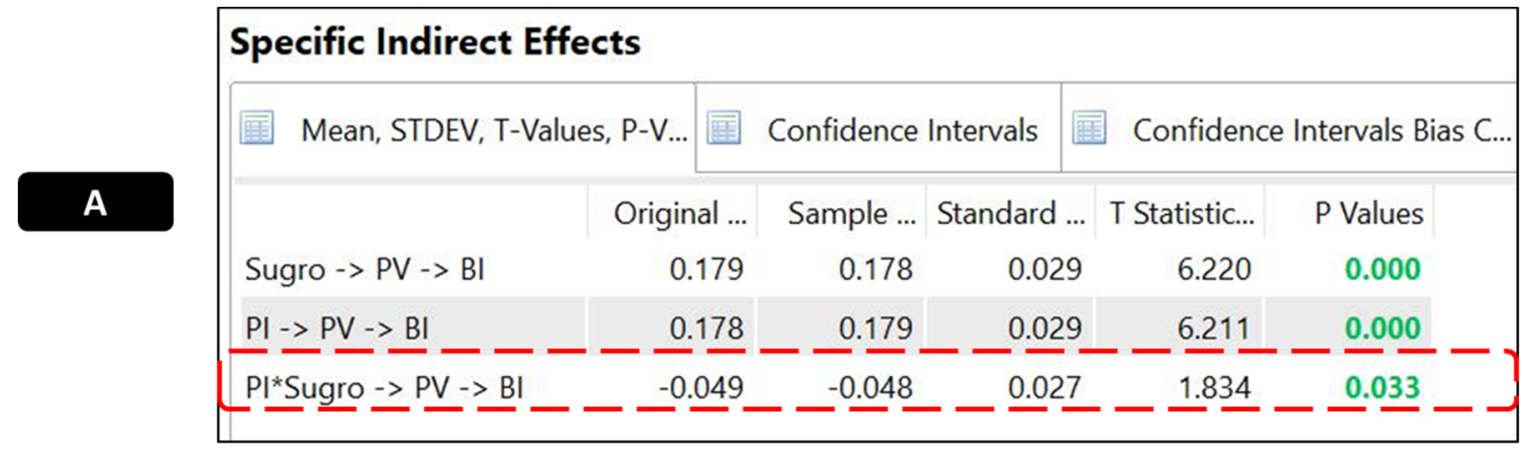

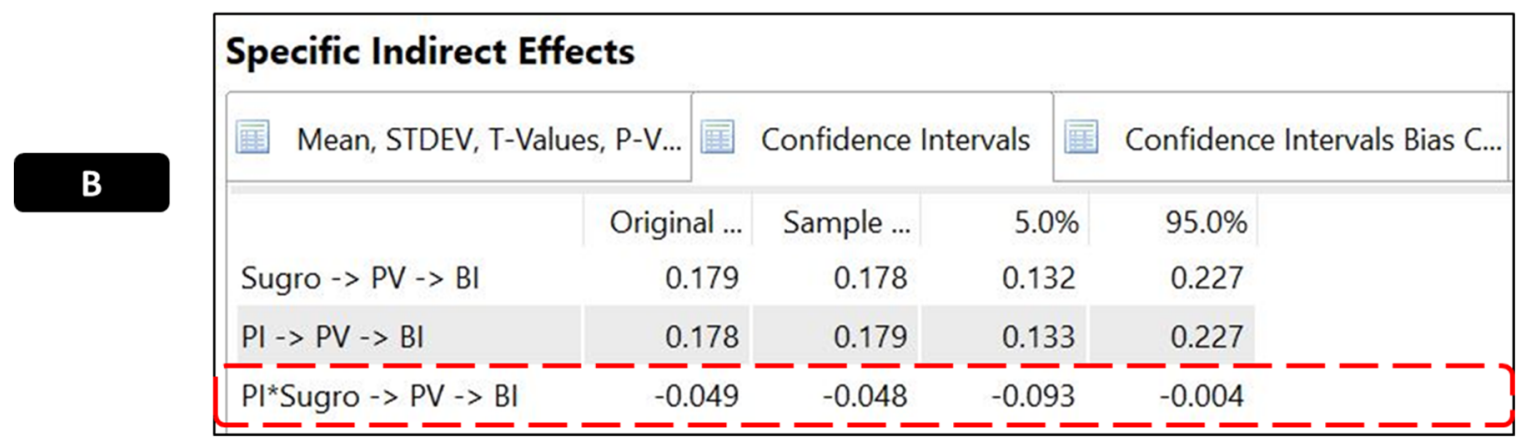

Figure B7. (Panel A) The p-value result for the index of " $p_{2} \cdot p_{5}$ " and (Panel B) the percentile-corrected result for the index of " $p_{2} \cdot p_{5}$ "

Next, we examine the mediated effects at different levels of the moderator. To formally test this effect for CoMe Model A, a researcher must be aware that there is no CoMe effects at low, medium, and high levels of the moderator as specified in the main body of the paper (see equations 6 to 8); rather, it requires them to insert the value of low SUGRO as 0 and high SUGRO as 1 into the formula of CoMe effects of Model A. Subsequently, using a spreadsheet application (i.e., MS Excel), we suggest carrying out the following steps:

1) Take the 10,000 path coefficients from all direct effects created by the bootstrap sampling and copy and paste them into a spreadsheet (see Figure B8). 


\begin{tabular}{|c|c|c|c|c|c|c|}
\hline$\Delta$ & A & B & C & D & $E$ & $\mathrm{~F}$ \\
\hline 1 & Original (O) & & & & & \\
\hline 2 & Percentile Lower (5\%) & & & & & \\
\hline 3 & Percentile Upper (95\%) & & & & & \\
\hline 4 & Standard Error & & & & & \\
\hline 5 & t-value & & & & & \\
\hline 6 & & & & & & \\
\hline 7 & & p1 & p2 & p3 & p4 & p5 \\
\hline 8 & & PI $->$ PV & PV $->$ BI & $\mathrm{PI}->\mathrm{BI}$ & SUGRO -> PV & PI*SUGRO -> PV \\
\hline 9 & Sample 0 & 0.347 & 0.505 & 0.255 & 0.344 & -0.197 \\
\hline 10 & Sample 1 & 0.304 & 0.547 & 0.194 & 0.412 & -0.058 \\
\hline 11 & Sample 2 & 0.489 & 0.440 & 0.311 & 0.293 & -0.069 \\
\hline 12 & Sample 3 & 0.365 & 0.462 & 0.320 & 0.370 & -0.024 \\
\hline 13 & Sample 4 & 0.409 & 0.487 & 0.320 & 0.343 & -0.127 \\
\hline 14 & Sample 5 & 0.409 & 0.481 & 0.327 & 0.323 & -0.068 \\
\hline
\end{tabular}

Figure B8. Step 1 Illustrative Example for Use of Categorical Moderator (CoMe Model A)

2) Based on columns $B$ to $F$, create a new column for the CoMe effect at low moderator level (or Low Sugro) and CoMe effect at high moderator level (or High Sugro) using the equations suggested in Figure B9.

Create each of these columns using the equations for the CoMe effects concerning Model A. Drawing on these equations enables obtaining the subsample results of CoMe effect at low moderator level (or Low Sugro) and CoMe effect at high moderator level (or High Sugro) when inserting the value of 0 and 1 respectively.

\begin{tabular}{|c|c|c|c|c|c|c|c|}
\hline$\triangle$ & $\mathrm{H}$ & 1 & $J$ & K & L & M & $\mathrm{N}$ \\
\hline 1 & Original (0) & & & & & & \\
\hline 2 & Lower Percentile (5\%) & & & & & & \\
\hline 3 & Upper Percentile (95\%) & & & & & & \\
\hline 4 & Standard Error & & & & & & \\
\hline 5 & t-value & & & & & & \\
\hline 6 & & -ー-ー-ー- & $-\pi-n-1$ & 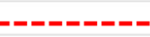 & 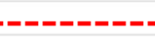 & 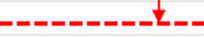 & Iーーーーーーーーーーー \\
\hline 7 & 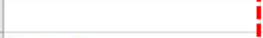 & & & & & LOW SUGRO (0) & HIGH SUGRO (1) \\
\hline 8 & Formula & p5* $(0-0)$ & $\mathrm{p} 5 *(0+1)$ & $p 1+p 5 *(0-0)$ & $p 1+p 5 *(0+1)$ & $\left(p 1+p 5^{*}(0-0)\right)^{*} p 2$ & $(p 1+p 5 *(0+1)) * p 2$ \\
\hline 9 & 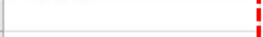 & 0.000 & -0.197 & 0.347 & 0.150 & 0.175 & 0.076 \\
\hline 10 & 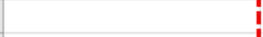 & 0.000 & -0.058 & 0.304 & 0.246 & 0.166 & 0.135 \\
\hline 11 & 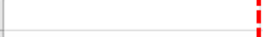 & 0.000 & -0.069 & 0.489 & 0.420 & 0.215 & 0.185 \\
\hline 12 & & 0.000 & -0.024 & 0.365 & 0.341 & 0.169 & 0.158 \\
\hline 13 & & 0.000 & -0.127 & 0.409 & _ 0.282 & - $\underline{0.199}$ & 0.137 \\
\hline
\end{tabular}

Figure B9. Step 2 Illustrative Example for Use of Categorical Moderator (CoMe Model A)

3) Calculate the original values $(\mathrm{O})$ provided by the PLS-SEM path coefficients for each subsample (see Figure B10). The results show that the path coefficient effects for CoMe effects of High Sugro are weaker than for Low Sugro. 
Calculate the original values (0) provided by PLS for path coefficients.

The details are: =AVERAGE(M9:M10008) and =AVERAGE(N9:N10008)

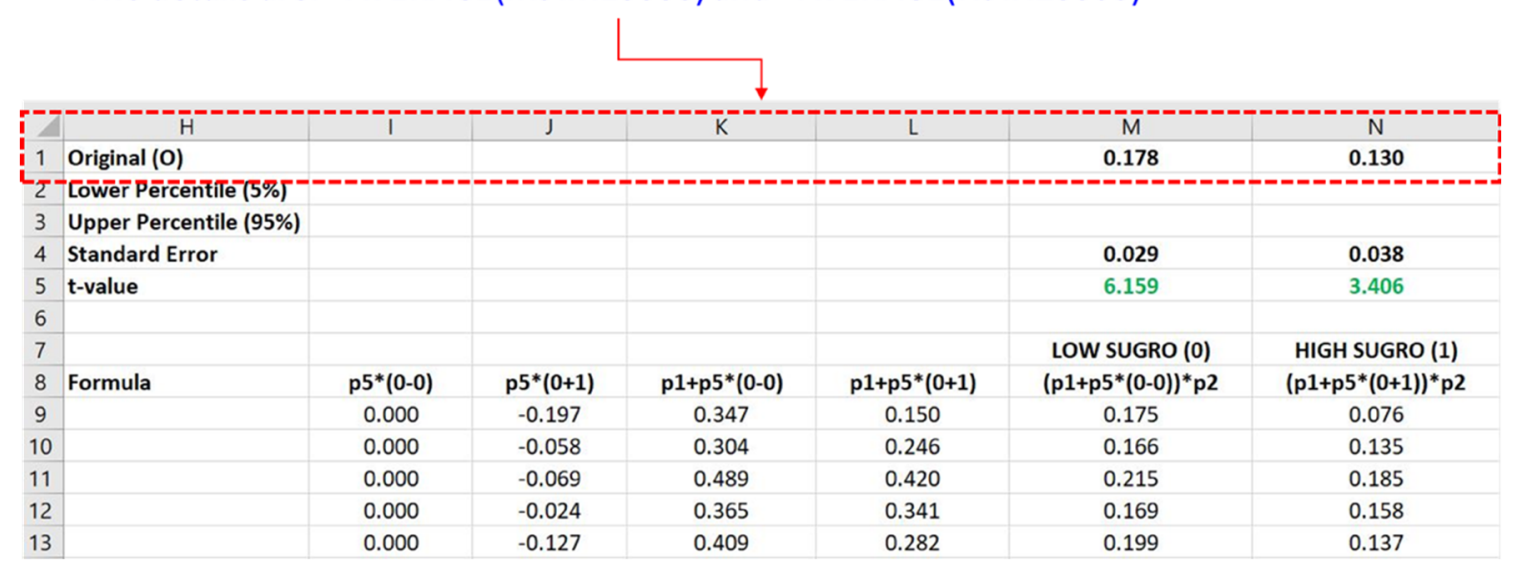

Figure B10. Step 3 Illustrative Example for Use of Categorical Moderator (CoMe Model A)

4) Estimate the percentile bootstrap confidence interval $(\mathrm{Cl})$ using the function PERCENTILE (range, $k$ ), where $\mathrm{k}$ is the percentile value between 0 and 1 (see Figure B11). If the $\mathrm{Cl}$ for the product "does not include a 0 value, this result means that the mediated effects at different levels of the moderator are significantly different from 0." 3 In our example, the CoMe effects for Low Sugro and High Sugro are significant because both the extremes of the $\mathrm{Cl}$ are positive. Therefore, we find some evidence to support the hypothesis of Model A; that is, the mediated effect of the consumer's PI on BI via PV reduces as SUGRO increases.

Calculate both the lower percentile (5\%) and the upper percentile (95\%).

- The details of the lower percentile for CoMe effect at low moderator level (or LOW SUGRO) and CoMe effect at high moderator level (or HIGH SUGRO) are: $=$ PERCENTILE(M9:M10008, 0.05) and =PERCENTILE(N9:N10008, 0.05)

- The details of the upper percentile for CoMe effect at low moderator level (or LOW SUGRO) and CoMe effect at high moderator level (or HIGH SUGRO) are: $=$ PERCENTILE(M9:M10008, 0.95) and =PERCENTILE(N9:N10008, 0.95)

\begin{tabular}{|c|c|c|c|c|c|c|c|}
\hline$\Delta$ & $\mathrm{H}$ & I & $\mathrm{J}$ & $K$ & L & M & $\mathrm{N}$ \\
\hline 1 & Original (0) & & & & & 0.178 & 0.130 \\
\hline 2 & Lower Percentile (5\%) & & & & & 0.132 & 0.071 \\
\hline 3 & Upper Percentile (95\%) & & & & & 0.227 & 0.196 \\
\hline 4 & Standard Error & & & & & 0.029 & 0.038 \\
\hline 5 & t-value & & & & & 6.159 & 3.406 \\
\hline \multicolumn{8}{|c|}{6} \\
\hline 7 & & & & & & LOW SUGRO (0) & HIGH SUGRO (1) \\
\hline 8 & Formula & $\mathrm{p} 5 *(0-0)$ & $\mathrm{p} 5 *(0+1)$ & $\mathrm{p} 1+\mathrm{p} 5 *(0-0)$ & $p 1+p 5 *(0+1)$ & $(p 1+p 5 *(0-0))^{*} p 2$ & $(\mathrm{p} 1+\mathrm{p} 5 *(0+1)) * \mathrm{p} 2$ \\
\hline 9 & & 0.000 & -0.197 & 0.347 & 0.150 & 0.175 & 0.076 \\
\hline 10 & & 0.000 & -0.058 & 0.304 & 0.246 & 0.166 & 0.135 \\
\hline 11 & & 0.000 & -0.069 & 0.489 & 0.420 & 0.215 & 0.185 \\
\hline 12 & & 0.000 & -0.024 & 0.365 & 0.341 & 0.169 & 0.158 \\
\hline 13 & & 0.000 & -0.127 & 0.409 & 0.282 & 0.199 & 0.137 \\
\hline
\end{tabular}

Figure B11. Step 4 Illustrative Example for Use of Categorical Moderator (CoMe Model A)

5) Finally, the researcher can create a graphical plot of the mediated effects at different levels of the moderator via an Excel spreadsheet (see Figure 10 in the main body of the paper).

${ }^{3}$ To calculate the standard error and t-value, researchers can consider the comments in Footnote 1 and Footnote 2 in this appendix. 


\section{Appendix C(i): Step-by-Step Procedure for Conditional Mediation Analysis (CoMe Model B) Using a Continuous Moderator}

Given that the index of " $p_{1} \cdot p_{5}$ " cannot be directly obtained from any type of PLS-SEM software (e.g., SmartPLS), we need to examine the results of the path coefficients using the output of the bootstrapping sample. Consequently, once we run the estimations, we next perform a bootstrapping procedure with 10,000 subsamples. Then, using a spreadsheet and multiplying the bootstrapping outputs (i.e., $p_{1} \cdot p_{5}$ ), we calculate the percentile bootstrap $\mathrm{Cl}$ for the index of " $p_{1} \cdot p_{5}$ ". Thus, using a spreadsheet application (i.e., MS Excel), we suggest carrying out the following steps:

1) Take the 10,000 path coefficients from all direct effects created by the bootstrap procedure and copy and paste them into a spreadsheet (Figure C1).

\begin{tabular}{|c|c|c|c|c|c|c|}
\hline$\Delta$ & A & B & C & D & $\mathrm{E}$ & $\mathrm{F}$ \\
\hline 1 & Original (0) & & & & & \\
\hline 2 & Percentile Lower (5\%) & & & & & \\
\hline 3 & Percentile Upper (95\%) & & & & & \\
\hline 4 & Standard Error & & & & & \\
\hline 5 & t-value & & & & & \\
\hline 6 & & & & & & \\
\hline 7 & & p1 & p2 & p3 & p4 & p5 \\
\hline 8 & & $\mathrm{PI} \rightarrow>\mathrm{PV}$ & $\mathrm{PV}->\mathrm{BI}$ & $\mathrm{PI}->\mathrm{BI}$ & SUGRO -> BI & $\mathrm{PV} *$ Sugro $->\mathrm{BI}$ \\
\hline 9 & Sample 0 & 0.543 & 0.341 & 0.208 & 0.291 & -0.046 \\
\hline 10 & Sample 1 & 0.55 & 0.309 & 0.14 & 0.377 & -0.036 \\
\hline 11 & Sample 2 & 0.52 & 0.277 & 0.217 & 0.338 & -0.041 \\
\hline 12 & Sample 3 & 0.548 & 0.325 & 0.259 & 0.21 & -0.055 \\
\hline 13 & Sample 4 & 0.517 & 0.438 & 0.114 & 0.261 & -0.068 \\
\hline
\end{tabular}

Figure C1. Step 1 Illustrative Example for Use of Continuous Moderator (CoMe Model B)

2) Create a new column for the index of " $p_{1} \cdot p_{5}$ " under assessment, and calculate the product of " $p_{1} \cdot p_{5}$ " (see Figure C2).

\begin{tabular}{|c|c|c|c|c|c|c|c|}
\hline$\Delta$ & A & B & C & D & $\mathrm{E}$ & $\mathrm{F}$ & G \\
\hline 1 & Original (0) & & & & & & \\
\hline 2 & Percentile Lower (5\%) & \multicolumn{6}{|c|}{ Create a new column "p1 * p5", and calculate } \\
\hline 3 & Percentile Upper (95\%) & \multicolumn{6}{|c|}{ the product of the paths that form the index } \\
\hline 4 & Standard Error & & & & \multicolumn{3}{|c|}{ under assessment } \\
\hline 5 & t-value & & & & & & \\
\hline 6 & & & & & & & \\
\hline 7 & & p1 & p2 & p3 & p4 & p5 & Index \\
\hline 8 & & $\mathrm{PI}->\mathrm{PV}$ & $\mathrm{PV}->\mathrm{BI}$ & $\mathrm{PI}->\mathrm{BI}$ & SUGRO -> BI & $\mathrm{PV}^{*}$ Sugro $->\mathrm{BI}$ & p1*p5 \\
\hline 9 & Sample 0 & 0.543 & 0.341 & 0.208 & 0.291 & -0.046 & -0.02498 \\
\hline 10 & Sample 1 & 0.55 & 0.309 & 0.14 & 0.377 & -0.036 & -0.0198 \\
\hline 11 & Sample 2 & 0.52 & 0.277 & 0.217 & 0.338 & -0.041 & -0.02132 \\
\hline 12 & Sample 3 & 0.548 & 0.325 & 0.259 & 0.21 & -0.055 & -0.03014 \\
\hline 13 & Sample 4 & 0.517 & 0.438 & 0.114 & 0.261 & -0.068 & -0.03516 \\
\hline
\end{tabular}

Figure C2. Step 2 Illustrative Example for Use of Continuous Moderator (CoMe Model B) 
3) Calculate the original values $(O)$ to obtain the index value of " $p_{1} \cdot p_{5}$ " (see Figure $C 3$ ).

Calculate the original values $(0)$ to obtain the index value of " $p 1$ * $p 5$ ".

The detail is: =AVERAGE(G9:G10008)

\begin{tabular}{|c|c|c|c|c|c|c|c|}
\hline$\Delta$ & A & B & C & D & $E$ & $\mathrm{~F}$ & ---_6_--- \\
\hline 1 & Original (0) & & & & & & -0.021 \\
\hline 2 & Percentile Lower (5\%) & & & & & & \\
\hline 3 & Percentile Upper (95\%) & & & & & & \\
\hline 4 & Standard Error & & & & & & 0.014 \\
\hline 5 & t-value & & & & & & -1.518 \\
\hline \multicolumn{8}{|l|}{6} \\
\hline 7 & & p1 & p2 & p3 & p4 & p5 & Index \\
\hline 8 & & $\mathrm{PI} \rightarrow \mathrm{PV}$ & $\mathrm{PV} \rightarrow \mathrm{BI}$ & $\mathrm{PI} \rightarrow \mathrm{BI}$ & SUGRO -> BI & PV*Sugro $\rightarrow \mathrm{BI}$ & p1*p5 \\
\hline 9 & Sample 0 & 0.543 & 0.341 & 0.208 & 0.291 & -0.046 & -0.02498 \\
\hline 10 & Sample 1 & 0.55 & 0.309 & 0.14 & 0.377 & -0.036 & -0.0198 \\
\hline 11 & Sample 2 & 0.52 & 0.277 & 0.217 & 0.338 & -0.041 & -0.02132 \\
\hline 12 & Sample 3 & 0.548 & 0.325 & 0.259 & 0.21 & -0.055 & -0.03014 \\
\hline 13 & Sample 4 & 0.517 & 0.438 & 0.114 & 0.261 & -0.068 & -0.03516 \\
\hline
\end{tabular}

Figure C3. Step 3 Illustrative Example for Use of Continuous Moderator (CoMe Model B)

4) Estimate the percentile bootstrap $\mathrm{Cl}$, particularly for column " $p_{1} \cdot p_{5}$ ", using the function PERCENTILE (range, $k$ ), where $\mathrm{k}$ is the percentile value between 0 and 1 . In our case, given that our hypothesized effect is postulated with direction, we use a one-sided test, and we estimate a $90 \% \mathrm{Cl}$ (Figure C4). If the $\mathrm{Cl}$ for the product of " $p_{1} \cdot p_{5}$ " does not include a 0 value, this result means that the CoMe index is significantly different from $0 .{ }^{4}$ In our example, the index " $p_{1} \cdot p_{5}$ " is insignificant because both extremes of the $\mathrm{Cl}$ have mixed negative and positive sign; thus, there is change in sign between them. Therefore, we do not find evidence to support the hypothesis of Model B; that is, the mediated effect of a consumer's PI on BI via PV does not appear to decrease as SUGRO increases.

4 To calculate the standard error and t-value, researchers can consider the comments in Footnote 1 and Footnote 2 in this appendix. 
Lower Percentile (5\%) for "p1 * p5" =PERCENTILE(G6:G10005; 0,05)

Upper Percentile (95\%) for "p1 * p5" =PERCENTILE(G6:G10005; 0,95)

\begin{tabular}{|c|c|c|c|c|c|c|c|}
\hline$\Delta$ & A & B & C & D & $\mathrm{E}$ & $\mathrm{F}$ & G \\
\hline 1 & Original (0) & & & & & & -0.021 \\
\hline 2 & Percentile Lower (5\%) & & & & & & -0.044 \\
\hline 3 & Percentile Upper (95\%) & & & & & & L_o.002 \\
\hline 4 & Standard Error & & & & & & 0.014 \\
\hline 5 & t-value & & & & & & -1.518 \\
\hline \multicolumn{8}{|l|}{6} \\
\hline 7 & & p1 & p2 & p3 & p4 & p5 & Index \\
\hline 8 & & $\mathrm{PI}>\mathrm{PV}$ & $\mathrm{PV} \rightarrow \mathrm{BI}$ & $\mathrm{PI} \rightarrow \mathrm{BI}$ & SUGRO $->\mathrm{BI}$ & PV*Sugro $->\mathrm{BI}$ & p1*p5 \\
\hline 9 & Sample 0 & 0.543 & 0.341 & 0.208 & 0.291 & -0.046 & -0.02498 \\
\hline 10 & Sample 1 & 0.55 & 0.309 & 0.14 & 0.377 & -0.036 & -0.0198 \\
\hline 11 & Sample 2 & 0.52 & 0.277 & 0.217 & 0.338 & -0.041 & -0.02132 \\
\hline 12 & Sample 3 & 0.548 & 0.325 & 0.259 & 0.21 & -0.055 & -0.03014 \\
\hline 13 & Sample 4 & 0.517 & 0.438 & 0.114 & 0.261 & -0.068 & -0.03516 \\
\hline
\end{tabular}

Figure C4. Step 4 Illustrative Example for Us of Continuous Moderator (CoMe Model B)

Next, we will continue to examine the mediated effects at different levels of the moderator. ${ }^{5}$ To formally test this effect for Model B, we initially assess the standard deviation of the moderator (SUGRO). The standard deviation for Sugro is 1.0011. Subsequently, we continue using MS Excel to execute the following steps.

5) Create a new column for the CoMe effects at low, medium, and high levels of the moderator using the illustrated formula suggested in Figure C5.

Create these columns using equations 9 to 11 in

Table 1 (see main body of paper). These equations

will enable obtaining subsample results for CoMe

effect at low moderator level, CoMe effect at

medium moderator level, and CoMe effect at high

moderator level.

\begin{tabular}{|c|c|c|c|c|c|c|c|c|c|c|}
\hline$\Delta$ & I & J & $\mathrm{K}$ & L & M & $\mathrm{N}$ & 0 & P & Q & $\mathbf{R}$ \\
\hline 1 & Original (0) & & & & & & & & & \\
\hline 2 & Lower Percentile (5\%) & & & & & & & & & \\
\hline 3 & Upper Percentile (95\%) & & & & & & & & & \\
\hline 4 & Standard Error & & & & & & & & & \\
\hline 5 & t-value & & & & & & & & & \\
\hline 6 & & - & - & $\pi$ & & & $t_{-}$ & -ーーーー & -ーーーー- & ーーーーーーーーー \\
\hline 7 & & & & & & & & Low CoMe & Medium CoMe & High CoMe \\
\hline 8 & Formula & $\mathrm{p} 5 *(0-1.0011)$ & $\mathrm{p} 5^{*}(0)$ & $\mathrm{p} 5{ }^{*}(0+1.0011)$ & $\mathrm{p} 2+\mathrm{p5} 5^{*}(0-1.0011)$ & $p 2+p 5^{*}(0)$ & $p 2+p 5^{*}(0+1.0011)$ & $\left(p 2+p 5^{*}(0-1.0011)\right)^{*} p 1$ & $\left(p 2+p 5^{*}(0)\right)^{*} p 1$ & $\left(p 2+p 5^{*}(0+1.0011)\right)^{*} p 1$ \\
\hline 9 & & 0.0460506 & 0 & -0.0460506 & 0.3870506 & 0.341 & 0.2949494 & 0.210168476 & 0.185163 & 0.160157524 \\
\hline 10 & & 0.0360396 & 0 & -0.0360396 & 0.3450396 & 0.309 & 0.2729604 & 0.18977178 & 0.16995 & 0.15012822 \\
\hline 11 & & 0.0410451 & 0 & -0.0410451 & 0.3180451 & 0.277 & 0.2359549 & 0.165383452 & 0.14404 & 0.122696548 \\
\hline 12 & & 0.0550605 & 0 & -0.0550605 & 0.3800605 & 0.325 & 0.2699395 & 0.208273154 & 0.1781 & 0.147926846 \\
\hline 13 & & 0.0680748 & 0 & -0.0680748 & 0.5060748 & 0.438 & 0.3699252 & 0.261640672 & 0.226446 & 0.191251328 \\
\hline
\end{tabular}

Figure C5. Step 5 Illustrative Example for Us of Continuous Moderator (CoMe Model B)

6) Calculate the original values $(O)$ provided by the PLS-SEM path coefficients for each subsample (see Figure C6). The results show that the path coefficient effects of the CoMe effect become weaker when the Sugro effect is one standard deviation above the mean.

\footnotetext{
${ }^{5}$ If the index of conditional mediation is nonsignifcant, researchers should stop assessing the different level of CoMe effects; rather, they should analyze moderation and mediation separately (see Figure 1 in main body of this paper). In our case, we continue the empirical illustration merely to show the steps on how to obtain the CoMe effects for different levels of the moderator.
} 
Calculate the original values $(\mathrm{O})$ provided by PLS for path coefficients.

The details are: $=$ AVERAGE(P9:P10008), =AVERAGE(Q9:Q10008), and

$=$ AVERAGE(R9:R10008)

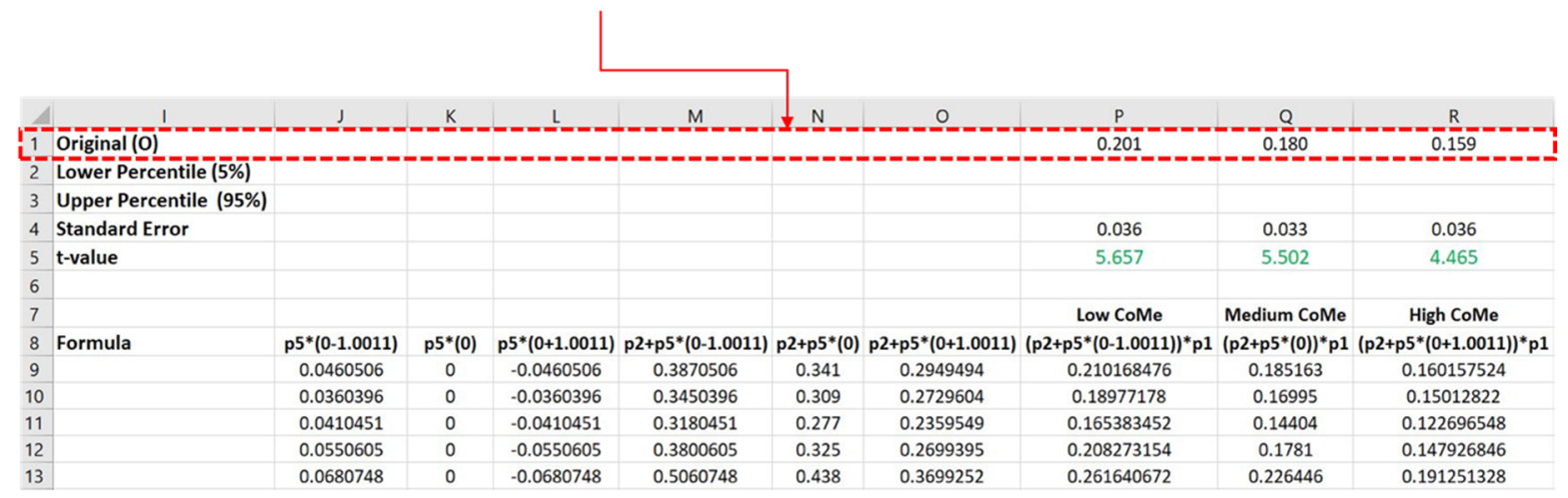

Figure C6. Step 6 Illustrative Example for Use of Continuous Moderator (CoMe Model B)

7) Estimate the percentile bootstrap $\mathrm{Cl}$ using the function PERCENTILE (range, $\mathrm{k}$ ), where $\mathrm{k}$ is the percentile value between 0 and 1 (see Figure $\mathrm{C} 7$ ). If the $\mathrm{Cl}$ for the product "does not include a 0 value, this result means that the mediated effects at different levels of the moderator are significantly different from $0 .{ }^{\prime 6}$ In our example, the CoMe effects at low, medium, and high levels of the moderator are significant because both extremes of the $\mathrm{Cl}$ are positive. However, we are unable to support the hypothesis of Model $\mathrm{B}$; that is, the mediated effect of PI on BI via PV is weaker due to the negative impact of SUGRO. The reason is that the index of conditional mediation is nonsignificant (see Figure C4). Thus, it is preferable to analyze and report moderation and mediation separately. Notably, researchers should not proceed with creating the graphical plot of the mediated effects at different levels of the moderator.

Calculate both the lower percentile (5\%) and the upper percentile (95\%).

- The details of the lower percentile for CoMe effect at low moderator level, CoMe effect at medium moderator level, and CoMe effect at high moderator level are:

$=$ PERCENTILE(P9:P10008, 0.05), =PERCENTILE(Q9:Q10008, 0.05), and

$=$ PERCENTILE(R9:R10008, 0.05)

- The details of the upper percentile for CoMe effect at low moderator level, CoMe effect at medium moderator level, and CoMe effect at high moderator level are: =PERCENTILE(P9:P10008, 0.95), =PERCENTILE(Q9:Q10008, 0.95), and $=$ PERCENTILE(R9:R10008, 0.95)

\begin{tabular}{|c|c|c|c|c|c|c|c|c|c|c|}
\hline$\Delta$ & 1 & J & $\mathrm{k}$ & L & M & $\mathrm{N}$ & 0 & P & Q & $\mathrm{R}$ \\
\hline \multicolumn{2}{|c|}{ 1_OriginalloL ___-_-_ } & -ーーーーーー- & --n & -ーー-ー-ーー & -ーーーーーーーー & $-\frac{1}{2}---$ & --ーーーーー-ー- & --n-0.201 - - - & -0.180 & - - 0.159 \\
\hline 2 & Lower Percentile (5\%) & & & & & & & 0.144 & 0.128 & 0.103 \\
\hline 3 & Upper Percentile (95\%) & & & & & & & 0.261 & 0.236 & 0.220 \\
\hline $4^{-}$ & 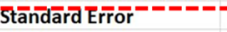 & & & & & & & $0 . \overline{036}$ & $=-\overline{0.033}=$ & $=-0.036-=-$ \\
\hline 5 & t-value & & & & & & & 5.657 & 5.502 & 4.465 \\
\hline \multicolumn{11}{|c|}{6} \\
\hline 7 & & & & & & & & Low CoMe & Medium CoMe & High CoMe \\
\hline 8 & Formula & $\mathrm{p} 5^{*}(0-1.0011)$ & $p 5^{*}(0)$ & $p 5^{*}(0+1.0011)$ & $p 2+p 5 *(0-1.0011)$ & $p 2+p 5^{*}(0)$ & $p 2+p 5 *(0+1.0011)$ & $(p 2+p 5 *(0-1.0011)) * p 1$ & $(p 2+p 5 *(0))^{*} p 1$ & $(p 2+p 5 *(0+1.0011))^{*} p 1$ \\
\hline 9 & & 0.0460506 & 0 & -0.0460506 & 0.3870506 & 0.341 & 0.2949494 & 0.210168476 & 0.185163 & 0.160157524 \\
\hline 10 & & 0.0360396 & 0 & -0.0360396 & 0.3450396 & 0.309 & 0.2729604 & 0.18977178 & 0.16995 & 0.15012822 \\
\hline 11 & & 0.0410451 & 0 & -0.0410451 & 0.3180451 & 0.277 & 0.2359549 & 0.165383452 & 0.14404 & 0.122696548 \\
\hline 12 & & 0.0550605 & 0 & -0.0550605 & 0.3800605 & 0.325 & 0.2699395 & 0.208273154 & 0.1781 & 0.147926846 \\
\hline 13 & & 0.0680748 & 0 & -0.0680748 & 0.5060748 & 0.438 & 0.3699252 & 0.261640672 & 0.226446 & 0.191251328 \\
\hline
\end{tabular}

Figure C7. Step 7 Illustrative example for use of continuous moderator (CoMe Model B)

${ }^{6}$ To calculate the standard error and t-value, researchers can consider the comments in Footnote 1 and Footnote 2 in this appendix. 


\section{Appendix C(ii): Step-by-Step Procedure for Conditional Mediation Analysis (CoMe Model B) When Moderator Is Converted from Continuous to Categorical}

Given that the index of " $p_{1} \cdot p_{5}$ " cannot be directly obtained from any type of PLS-SEM software, we need to examine the results of the path coefficients using the output of the bootstrapping sample, even when continuous data has been transformed to categorical data via median split. Consequently, once we estimate the model, we next perform a bootstrapping procedure with 10,000 subsamples. Then, using a spreadsheet and multiplying the bootstrapping outputs (i.e., $p_{1} \cdot p_{5}$ ), we calculate the percentile bootstrap $\mathrm{Cl}$ for the index of " $p_{1} \cdot p_{5}$ ". Thus, using an Excel spreadsheet, we suggest carrying out the following steps:

1) Take the 10,000 path coefficients from all direct effects created by the bootstrap procedure and copy and paste them into a spreadsheet (Figure C8).

\begin{tabular}{|c|c|c|c|c|c|c|c|}
\hline$\angle$ & A & B & C & D & E & $\mathrm{F}$ & G \\
\hline 1 & Original (0) & & & & & & \\
\hline 2 & Percentile Lower (5\%) & & & & & & \\
\hline 3 & Percentile Upper (95\%) & & & & & & \\
\hline 4 & Standard Error & & & & & & \\
\hline 5 & t-value & & & & & & \\
\hline \multicolumn{8}{|l|}{6} \\
\hline 7 & & p1 & p2 & p3 & p4 & p5 & Index \\
\hline 8 & & $\mathrm{PI} \rightarrow \mathrm{PV}$ & $\mathrm{PV} \rightarrow \mathrm{BI}$ & $\mathrm{PI} \rightarrow \mathrm{BI}$ & Sugro $->B$ & PV*Sugro $\rightarrow \mathrm{BI}$ & $\mathrm{p} 1 * \mathrm{p} 5$ \\
\hline 9 & Sample 0 & 0.543 & 0.412 & 0.232 & 0.115 & -0.085 & -0.046155 \\
\hline 10 & Sample 1 & 0.522 & 0.312 & 0.248 & 0.229 & -0.181 & -0.094482 \\
\hline 11 & Sample 2 & 0.45 & 0.455 & 0.166 & 0.137 & -0.239 & -0.10755 \\
\hline 12 & Sample 3 & 0.554 & 0.391 & 0.26 & 0.207 & -0.185 & -0.10249 \\
\hline 13 & Sample 4 & 0.488 & 0.47 & 0.183 & 0.173 & -0.212 & -0.103456 \\
\hline 14 & Sample 5 & 0.559 & 0.313 & 0.352 & 0.198 & -0.091 & -0.050869 \\
\hline
\end{tabular}

Figure C8. Step 1 Illustrative Example for Use of Categorical Moderator (CoMe Model B)

2) Create a new column for the index of " $p_{1} \cdot p_{5}$ " under assessment, and calculate the product of " $p_{1} \cdot p_{5}$ " (see Figure C9). 


\begin{tabular}{|c|c|c|c|c|c|c|c|}
\hline$\Delta$ & A & B & C & D & $E$ & $\mathrm{~F}$ & G \\
\hline 1 & Original (0) & & & & & & \\
\hline 2 & Percentile Lower (5\%) & \multirow{2}{*}{\multicolumn{6}{|c|}{ Create a new column "p1 * p5", and calculate }} \\
\hline 3 & Percentile Upper (95\%) & & & & & & \\
\hline 4 & Standard Error & & & & \multirow{2}{*}{\multicolumn{3}{|c|}{ under assessment }} \\
\hline 5 & t-value & & & & & & \\
\hline 6 & & & & & & & \\
\hline 7 & & p1 & p2 & p3 & p4 & p5 & Index \\
\hline 8 & & $\mathrm{PI} \rightarrow>\mathrm{PV}$ & $\mathrm{PV} \rightarrow \mathrm{BI}$ & $\mathrm{PI} \rightarrow \mathrm{BI}$ & \multicolumn{2}{|c|}{ Sugro $->$ B PV*Sugro $->\mathrm{BI}$} & $\mathrm{p} 1 * \mathrm{p} 5$ \\
\hline 9 & Sample 0 & 0.543 & 0.412 & 0.232 & 0.115 & -0.085 & -0.046155 \\
\hline 10 & Sample 1 & 0.522 & 0.312 & 0.248 & 0.229 & -0.181 & -0.094482 \\
\hline 11 & Sample 2 & 0.45 & 0.455 & 0.166 & 0.137 & -0.239 & -0.10755 \\
\hline 12 & Sample 3 & 0.554 & 0.391 & 0.26 & 0.207 & -0.185 & -0.10249 \\
\hline 13 & Sample 4 & 0.488 & 0.47 & 0.183 & 0.173 & -0.212 & -0.103456 \\
\hline 14 & Sample 5 & 0.559 & 0.313 & 0.352 & 0.198 & -0.091 & -0.050869 \\
\hline
\end{tabular}

Figure C9. Step 2 Illustrative Example for Use of Categorical Moderator (CoMe Model B)

3) Calculate the original values $(\mathrm{O})$ to obtain the index value of " $p_{1} \cdot p_{5}$ " (see Figure $\mathrm{C} 10$ ).

Calculate the original values $(0)$ to obtain the index value of "p1 * p5".

The detail is: =AVERAGE(G9:G10008)

\begin{tabular}{|c|c|c|c|c|c|c|c|}
\hline$\Delta$ & A & B & C & D & $\mathrm{E}$ & $\mathrm{F}$ & 5 \\
\hline 1 & Original (O) & & & & & & -0.078 \\
\hline 2 & Percentile Lower (5\%) & & & & & & \\
\hline 3 & Percentile Upper (95\%) & & & & & & \\
\hline 4 & Standard Error & & & & & & 0.024 \\
\hline 5 & t-value & & & & & & -3.294 \\
\hline \multicolumn{8}{|l|}{6} \\
\hline 7 & & p1 & p2 & p3 & p4 & p5 & Index \\
\hline 8 & & $\mathrm{PI} \rightarrow \mathrm{PV}$ & $\mathrm{PV} \rightarrow \mathrm{BI}$ & $\mathrm{PI} \rightarrow \mathrm{BI}$ & Sugro $->B$ & PV*Sugro -> BI & p1*p5 \\
\hline 9 & Sample 0 & 0.543 & 0.412 & 0.232 & 0.115 & -0.085 & -0.046155 \\
\hline 10 & Sample 1 & 0.522 & 0.312 & 0.248 & 0.229 & -0.181 & -0.094482 \\
\hline 11 & Sample 2 & 0.45 & 0.455 & 0.166 & 0.137 & -0.239 & -0.10755 \\
\hline 12 & Sample 3 & 0.554 & 0.391 & 0.26 & 0.207 & -0.185 & -0.10249 \\
\hline 13 & Sample 4 & 0.488 & 0.47 & 0.183 & 0.173 & -0.212 & -0.103456 \\
\hline
\end{tabular}

Figure C10. Step 3 Illustrative Example for Use of Categorical Moderator (CoMe Model B)

4) Estimate the percentile bootstrap $\mathrm{Cl}$, particularly for column " $p_{1} \cdot p_{5}$ ", using the function PERCENTILE (range, $k$ ), where $k$ is the percentile value between 0 and 1 . In our case, given that our hypothesized effect is postulated with direction, we use a one-sided test, and we estimate a $90 \% \mathrm{Cl}$ (Figure C11). If the Cl for 
the product of " $p_{1} \cdot p_{5}$ " does not include a 0 value, this result means that the conditional mediating effect is significantly different from $0 .{ }^{7}$ In our example, the index " $p_{1} \cdot p_{5}$ " is significant because both extremes of the $\mathrm{Cl}$ have negative sign, thus, there is change in sign between them. Therefore, we do find evidence to support the hypothesis of Model B; that is, the mediated effect of a consumer's PI on BI via PV reduces as SUGRO increases.

Lower Percentile (5\%) for "p1 * p5" =PERCENTILE(G9:G10008; 0,05)

Upper Percentile (95\%) for " $\mathrm{p} 1$ * p5" =PERCENTILE(G9:G10008; 0,95)

\begin{tabular}{|c|c|c|c|c|c|c|c|}
\hline$\Delta$ & A & B & C & D & $E$ & $\mathrm{~F}$ & G \\
\hline 1 & Original (O) & & & & & & -0.078 \\
\hline 2 & Percentile Lower (5\%) & & & & & & -0.117 \\
\hline 3 & Percentile Upper (95\%) & & & & & & -0.040 \\
\hline 4 & Standard Error & & & & & & 0.024 \\
\hline 5 & t-value & & & & & & -3.294 \\
\hline \multicolumn{8}{|l|}{6} \\
\hline 7 & & p1 & p2 & p3 & p4 & p5 & Index \\
\hline 8 & & $\mathrm{PI} \rightarrow \mathrm{PV}$ & $\mathrm{PV} \rightarrow \mathrm{BI}$ & $\mathrm{PI} \rightarrow \mathrm{BI}$ & Sugro $\rightarrow B$ & PV*Sugro $->\mathrm{BI}$ & p1*p5 \\
\hline 9 & Sample 0 & 0.543 & 0.412 & 0.232 & 0.115 & -0.085 & -0.046155 \\
\hline 10 & Sample 1 & 0.522 & 0.312 & 0.248 & 0.229 & -0.181 & -0.094482 \\
\hline 11 & Sample 2 & 0.45 & 0.455 & 0.166 & 0.137 & -0.239 & -0.10755 \\
\hline 12 & Sample 3 & 0.554 & 0.391 & 0.26 & 0.207 & -0.185 & -0.10249 \\
\hline 13 & Sample 4 & 0.488 & 0.47 & 0.183 & 0.173 & -0.212 & -0.103456 \\
\hline
\end{tabular}

Figure C11. Step 4 Illustrative Example for Use of Categorical Moderator (CoMe Model B)

5) Create a new column for values concerning CoMe effects at low (or Low Sugro) and CoMe effects at high (or High Sugro) levels of moderator using the illustrated formula suggested in Figure C12.

\footnotetext{
7 To calculate the standard error and t-value, researchers can consider the comments in Footnote 1 and Footnote 2 in this appendix.
} 
Create values for each column using Model B's equation of the CoMe effects. This will enable obtaining subsample results for CoMe effect at low moderator level (or Low Sugro) and CoMe effect at high moderator level (or High Sugro) when inserting the value of 0 and 1 respectively.

\begin{tabular}{|c|c|c|c|c|c|c|c|}
\hline 4 & 1 & $J$ & K & $\mathrm{L}$ & M & $\mathrm{N}$ & $\mathrm{O}$ \\
\hline 1 & Original (O) & & & & & & \\
\hline 2 & Lower Percentile (5\%) & & & & & & \\
\hline 3 & Upper Percentile (95\%) & & & & & & \\
\hline 4 & Standard Error & & & & & & \\
\hline 5 & t-value & & & & & & \\
\hline 6 & & & & & & & \\
\hline 7 & & & & & & Low SUGRO & High SUGRO \\
\hline 8 & Formula & p5*(0) & p5*(1) & $p 2+p 5 *(0)$ & $p 2+p 5 *(1)$ & $(p 2+p 5 *(0)) * p 1$ & $(p 2+p 5 *(0+1)) * p 1$ \\
\hline 9 & & 0 & -0.085 & 0.412 & 0.327 & 0.223716 & 0.177561 \\
\hline 10 & & 0 & -0.181 & 0.312 & 0.131 & 0.162864 & 0.068382 \\
\hline 11 & & 0 & -0.239 & 0.455 & 0.216 & 0.20475 & 0.0972 \\
\hline 12 & & 0 & -0.185 & 0.391 & 0.206 & 0.216614 & 0.114124 \\
\hline 13 & & 0 & -0.212 & 0.47 & 0.258 & 0.22936 & 0.125904 \\
\hline
\end{tabular}

Figure C12. Step 5 Illustrative example for use of categorical moderator (CoMe Model B)

6) Calculate the original values $(O)$ provided by the PLS-SEM path coefficients for each subsample (see Figure C13). The results show that the path coefficient effects for CoMe effects of high Sugro become weaker than for low Sugro.

Calculate the original values $(0)$ provided by PLS for path coefficients.

The details are: =AVERAGE(N9:N10008) and =AVERAGE(09:010008)

\begin{tabular}{|c|c|c|c|c|c|c|c|}
\hline-1 & --_- & ــــ & -_- & _t__L__- & $\underline{M}$ & -_N & _-_으... \\
\hline 1 & Original (0) & & & & & 0.212 & 0.134 \\
\hline 2 & Lower Percentile $(5 \%)$ & & & & & & \\
\hline 3 & Upper Percentile (95\%) & & & & & & \\
\hline 4 & Standard Error & & & & & 0.033 & 0.038 \\
\hline 5 & t-value & & & & & 6.504 & 3.559 \\
\hline \multicolumn{8}{|l|}{6} \\
\hline 7 & & & & & & Low SUGRO & High SUGRO \\
\hline 8 & Formula & $\mathrm{p} 5 *(0)$ & p5*(1) & $p 2+p 5^{*}(0)$ & $p 2+p 5 *(1)$ & $(p 2+p 5 *(0))^{*} p 1$ & $(p 2+p 5 *(0+1)) * p 1$ \\
\hline 9 & & 0 & -0.085 & 0.412 & 0.327 & 0.223716 & 0.177561 \\
\hline 10 & & 0 & -0.181 & 0.312 & 0.131 & 0.162864 & 0.068382 \\
\hline 11 & & 0 & -0.239 & 0.455 & 0.216 & 0.20475 & 0.0972 \\
\hline 12 & & 0 & -0.185 & 0.391 & 0.206 & 0.216614 & 0.114124 \\
\hline 13 & & 0 & -0.212 & 0.47 & 0.258 & 0.22936 & 0.125904 \\
\hline
\end{tabular}

Figure C13. Step 6 Illustrative Example for Use of Categorical Moderator (CoMe Model B) 
7) Estimate the percentile bootstrap confidence interval $(\mathrm{Cl})$ using the function PERCENTILE (range, $\mathrm{k}$ ), where $\mathrm{k}$ is the percentile value between 0 and 1 (see Figure $\mathrm{C14}$ ). If the $\mathrm{Cl}$ for the product "does not include a 0 value, this result means that the mediated effects at different levels of the moderator are significantly different from 0 ." In our example, the CoMe effects for low Sugro and high Sugro are significant because both extremes of the $\mathrm{Cl}$ are positive. Therefore, we find some evidence to support the hypothesis of Model B; that is, the mediated effect of the consumer's PI on BI via PV reduces as SUGRO increases.

Calculate both the lower percentile (5\%) and the upper percentile (95\%).

- The details of the lower percentile for CoMe effect at low moderator level (or Low Sugro) and CoMe effect at high moderator level (or High Sugro) are: =PERCENTILE(N9:N10008, 0.05) and =PERCENTILE(09:010008, 0.05)

- The details of the upper percentile for CoMe effect at low moderator level (or Low Sugro) and CoMe effect at high moderator level (or High Sugro) are: =PERCENTILE(N9:N10008, 0.95) and =PERCENTILE(O9:010008, 0.95)

\begin{tabular}{|c|c|c|c|c|c|c|c|}
\hline$\Delta$ & 1 & J & $\mathrm{K}$ & $\square$ & M & $\mathrm{N}$ & 0 \\
\hline 1 & Original $(0)$ & & & & & 0.212 & 0.134 \\
\hline$\overline{2}$ & Lower Percentile (5\%) & & & & & $0 . \overline{159}$ & $0.073^{-}$ \\
\hline 3 & _Upper_Percentile_(95\%) & & & & & -_o.2믄_-_ & | \\
\hline 4 & Standard Error & & & & & 0.033 & 0.038 \\
\hline 5 & t-value & & & & & 6.504 & 3.559 \\
\hline 6 & & & & & & & \\
\hline 7 & & & & & & Low SUGRO & High SUGRO \\
\hline 8 & Formula & p5*(0) & $\mathrm{p} 5 *(1)$ & $p 2+p 5 *(0)$ & $\mathrm{p} 2+\mathrm{p} 5 *(1)$ & $\left(p 2+p 5^{*}(0)\right)^{*} p 1$ & $\left(p 2+p 5^{*}(0+1)\right)^{*} p 1$ \\
\hline 9 & & 0 & -0.085 & 0.412 & 0.327 & 0.223716 & 0.177561 \\
\hline 10 & & 0 & -0.181 & 0.312 & 0.131 & 0.162864 & 0.068382 \\
\hline 11 & & 0 & -0.239 & 0.455 & 0.216 & 0.20475 & 0.0972 \\
\hline 12 & & 0 & -0.185 & 0.391 & 0.206 & 0.216614 & 0.114124 \\
\hline 13 & & 0 & -0.212 & 0.47 & 0.258 & 0.22936 & 0.125904 \\
\hline
\end{tabular}

Figure C14. Step 7 Illustrative Example for Use of Categorical Moderator (CoMe Model B)

8) Finally, the researcher can create a graphical plot of the mediated effects at different levels of the moderator via an Excel spreadsheet (see Figure 12 in the main body of the paper). 


\section{Appendix D(i). Step-by-Step Procedure for Conditional Mediation Analysis (CoMe Model C) for continuous moderator}

Given that the index of " $p_{2} \cdot p_{5}$ " can be obtained except for " $p_{1} \cdot p_{7}$ ", we decided to examine the results of the path coefficients using the output of the bootstrapping sample. Consequently, once we estimate the model, we next perform a bootstrapping procedure with 10,000 subsamples. Then, using a spreadsheet and multiplying the bootstrapping outputs, we calculate the percentile bootstrap $\mathrm{Cl}$ for the index of " $p_{2} \cdot p_{5}$ " and " $p_{1} \cdot p_{7}$ ". Subsequently, we estimate the index of CoMe Model $C$ by estimating the sum of both index of " $p_{2} \cdot p_{5}$ " and " $p_{1} \cdot p_{7}$ ". Thus, using an excel spreadsheet, we suggest carrying out the following steps:

1) Take the 10,000 path coefficients from all direct effects created by the bootstrap procedure and copy and paste them into a spreadsheet (Figure D1).

\begin{tabular}{|c|c|c|c|c|c|c|c|c|}
\hline 4 & A & B & c & D & E & $\mathrm{F}$ & G & $\mathrm{H}$ \\
\hline 1 & & & & & & & & \\
\hline 2 & Original (0) & & & & & & & \\
\hline 3 & Lower Percentile (5\%) & & & & & & & \\
\hline 4 & Upper Percentile (95\%) & & & & & & & \\
\hline 5 & Standard Error & & & & & & & \\
\hline 6 & t-value & & & & & & & \\
\hline 7 & & p1 & p2 & p3 & p4 & p5 & p6 & p7 \\
\hline 8 & & $\mathrm{PI} \rightarrow \mathrm{PV}$ & $P V \rightarrow B I$ & $\mathrm{PI}->\mathrm{BI}$ & SUGRO -> PV & PI*SUGRO $>$ PV & SUGRO $->\mathrm{BI}$ & PV*SUGRO $->\mathrm{BI}$ \\
\hline 9 & Sample 0 & 0.168 & 0.305 & 0.207 & 0.506 & -0.092 & 0.301 & -0.02 \\
\hline 10 & Sample 1 & 0.263 & 0.349 & 0.23 & 0.44 & -0.046 & 0.246 & -0.029 \\
\hline 11 & Sample 2 & 0.251 & 0.388 & 0.246 & 0.491 & -0.092 & 0.202 & -0.015 \\
\hline 12 & Sample 3 & 0.229 & 0.406 & 0.197 & 0.481 & -0.062 & 0.107 & -0.081 \\
\hline 13 & Sample 4 & 0.209 & 0.268 & 0.215 & 0.514 & -0.064 & 0.307 & -0.079 \\
\hline
\end{tabular}

Figure D1. Step 1 Illustrative Example for Use of Continuous Moderator (CoMe Model C)

2) Create a column for both the index of " $p_{2} \cdot p_{5}$ ", " $p_{1} \cdot p_{7}$ ", and " $p_{2} \cdot p_{5}+p_{1} \cdot p_{7}$ " as well as calculate the product of the paths that form the index under assessment (see Figure D2).

Create a new column "p2 * p5" "p1* p7", and

"p2 *p5 + p1*p7" as well as calculate the

product of the paths that form the index under

assessment

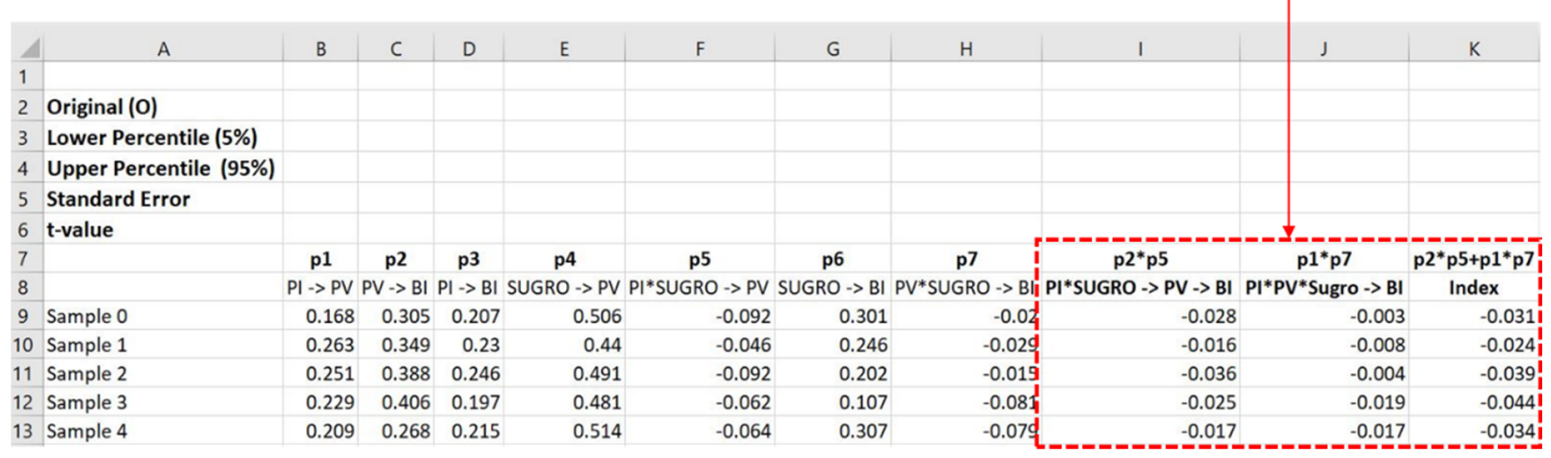

Figure D2. Step 2 Illustrative Example for Use of Continuous Moderator (CoMe Model C)

3) Calculate the original values $(\mathrm{O})$ to obtain the index value of " $p_{2} \cdot p_{5}$ ", " $p_{1} \cdot p_{7}$ ", and " $p_{2} \cdot p_{5}+p_{1} \cdot p_{7}$ " (see Figure D3). 
Calculate the original values $(0)$ to obtain the index value of "p2 * $\mathrm{p} 5$ ", "p1 * p7", and "p2* p5 + p1 * p7".

The detail are: =AVERAGE(19:I10008), =AVERAGE(J9:J10008), and $=$ AVERAGE(K9:K10008)

\begin{tabular}{|c|c|c|c|c|c|c|c|c|c|c|c|}
\hline 4 & $A$ & B & C & D & E & $\mathrm{F}$ & G & H & 1 & J & K \\
\hline \multicolumn{12}{|l|}{1} \\
\hline 2 & Original $(0)$ & & & & & & & & -0.026 & -0.009 & -0.035 \\
\hline 3 & Lower Percentile (5\%) & & & & & & & & & & \\
\hline 4 & Upper Percentile (95\%) & & & & & & & & & & \\
\hline 5 & Standard Error & & & & & & & & 0.012 & 0.006 & 0.013 \\
\hline 6 & $t$-value & & & & & & & & -2.153 & -1.397 & -2.658 \\
\hline 7 & & p1 & p2 & p3 & p4 & p5 & p6 & p7 & $\mathrm{p} 2{ }^{*} \mathrm{p} 5$ & p1*p7 & $p 2 * p 5+p 1^{*} p 7$ \\
\hline 8 & & $\mathrm{PI}->\mathrm{PV}$ & $\mathrm{PV}->\mathrm{BI}$ & $\mathrm{PI} \rightarrow \mathrm{BI}$ & SUGRO $>$ PV & PI*SUGRO -> PV & SUGRO $->\mathrm{BI}$ & PV*SUGRO ->BI & PI*SUGRO $->$ PV $>>B I$ & PI*PV*Sugro -> BI & Index \\
\hline 9 & Sample 0 & 0.168 & 0.305 & 0.207 & 0.506 & -0.092 & 0.301 & -0.02 & -0.028 & -0.003 & -0.031 \\
\hline 10 & Sample 1 & 0.263 & 0.349 & 0.23 & 0.44 & -0.046 & 0.246 & -0.029 & -0.016 & -0.008 & -0.024 \\
\hline 11 & Sample 2 & 0.251 & 0.388 & 0.246 & 0.491 & -0.092 & 0.202 & -0.015 & -0.036 & -0.004 & -0.039 \\
\hline 12 & Sample 3 & 0.229 & 0.406 & 0.197 & 0.481 & -0.062 & 0.107 & -0.081 & -0.025 & -0.019 & -0.044 \\
\hline 13 & Sample 4 & 0.209 & 0.268 & 0.215 & 0.514 & -0.064 & 0.307 & -0.079 & -0.017 & -0.017 & -0.034 \\
\hline
\end{tabular}

Figure D3. Step 3 Illustrative Example for Use of Continuous Moderator (CoMe Model C)

4) Estimate the percentile bootstrap $\mathrm{Cl}$, particularly for columns " $p_{2} \cdot p_{5}+p_{1} \cdot p_{7}$ ", using the function PERCENTILE (range, $k$ ), where $k$ is the percentile value between 0 and 1 . In our case, given that our hypothesis postulates a direction, we use a one-sided test, and we estimate a $90 \% \mathrm{Cl}$ (Figure D4). If the $\mathrm{Cl}$ for the product of " $p_{2} \cdot p_{5}+p_{1} \cdot p_{7}$ " does not include a 0 value, this result means that the conditional mediating effect is significantly different from $0 .{ }^{8}$ In our example, the index " $p_{2} \cdot p_{5}+p_{1} \cdot p_{7}$ " is significant because both extremes of the $\mathrm{Cl}$ have negative sign, thus, there is a change in sign between them. Therefore, we do find evidence to support the hypothesis of CoMe Model C; that is, the mediated effect of a consumer's PI on BI via PV reduces as SUGRO increases, even though " $p_{1} \cdot p_{7}$ " is insignificant.

Lower Percentile (5\%) for " $\mathrm{p} 2$ * p5" =PERCENTILE(I9:I10008; 0,05)

Upper Percentile (95\%) for "p2 * p5" =PERCENTILE(I9:I10008; 0,95)

Lower Percentile (5\%) for "p1 * p7" =PERCENTILE(J9:J10008; 0,05)

Upper Percentile (95\%) for "p1 * p7" =PERCENTILE(J9:J10008; 0,95)

Lower Percentile (5\%) for "p2 * p5 + p1 * p7" =PERCENTILE(K9:K10008; 0,05)

Upper Percentile (95\%) for "p1* $p 5+p 1 * p 7$ " =PERCENTILE(K9:K10008; 0,95)

\begin{tabular}{|c|c|c|c|c|c|c|c|c|c|c|c|}
\hline$\Delta$ & A & B & c & D & E & $\mathrm{F}$ & G & H & I & J & $\mathrm{K}$ \\
\hline \multicolumn{12}{|l|}{1} \\
\hline 2 & Original (0) & & & & & & & & $r----0.026---n$ & $---1-0,009=--1$ & $--0.035=-$ \\
\hline 3 & Lower Percentile (5\%) & & & & & & & & -0.046 & -0.020 & -0.057 \\
\hline 4 & Upper Percentile (95\%) & & & & & & & & -0.007 & 0.001 & | \\
\hline 5 & Standard Error & & & & & & & & 0.012 & 0.006 & 0.013 \\
\hline 6 & t-value & & & & & & & & -2.153 & -1.397 & -2.658 \\
\hline 7 & & p1 & p2 & p3 & p4 & p5 & p6 & p7 & p2*p5 & $p 1 * p 7$ & $p 2 * p 5+p 1 * p 7$ \\
\hline 8 & & $\mathrm{PI} \rightarrow \mathrm{PV}$ & $\mathrm{PV} \rightarrow \mathrm{BI}$ & $\mathrm{PI} \rightarrow \mathrm{BI}$ & SUGRO $>$ PV & PI*SUGRO -> PV & SUGRO $\rightarrow \mathrm{BI}$ & PV*SUGRO $>$ BI & PI*SUGRO -> PV $>$ BI & PI*PV*Sugro $\rightarrow$ BI & Index \\
\hline 9 & Sample 0 & 0.168 & 0.305 & 0.207 & 0.506 & -0.092 & 0.301 & -0.02 & -0.028 & -0.003 & -0.031 \\
\hline 10 & Sample 1 & 0.263 & 0.349 & 0.23 & 0.44 & -0.046 & 0.246 & -0.029 & -0.016 & -0.008 & -0.024 \\
\hline 11 & Sample 2 & 0.251 & 0.388 & 0.246 & 0.491 & -0.092 & 0.202 & -0.015 & -0.036 & -0.004 & -0.039 \\
\hline 12 & Sample 3 & 0.229 & 0.406 & 0.197 & 0.481 & -0.062 & 0.107 & -0.081 & -0.025 & -0.019 & -0.044 \\
\hline 13 & Sample 4 & 0.209 & 0.268 & 0.215 & 0.514 & -0.064 & 0.307 & -0.079 & -0.017 & -0.017 & -0.034 \\
\hline
\end{tabular}

Figure D4. Step 4 Illustrative example for use of continuous moderator (CoMe Model C)

8 To calculate the standard error and t-value, researchers can consider Footnote 1 and Footnote 2 in this appendix. 
Next, we will continue to examine the mediated effects at different levels of the moderator. To formally test this effect for CoMe Model C, we initially assess the standard deviation of the moderator (SUGRO). The standard deviation for Sugro is 1.0012. Subsequently, we continue using MS Excel to execute the following steps.

5) Create a new column for the CoMe effects at low (or low Sugro), medium (or medium Sugro), and high (or high Sugro) levels of the moderator using the formula suggested in Figure D5 to Figure D7.

Create values for columns using Equation 12

in Table 1 (see main body in paper). This

enables obtaining the subsample results for CoMe effect at low moderator level (or Low Sugro).

\begin{tabular}{|c|c|c|c|c|}
\hline$\Delta$ & M & $\mathrm{N}$ & 0 & $\mathrm{P}$ \\
\hline 1 & & & & Low Sugro \\
\hline 2 & Original (0) & & & \\
\hline 3 & Lower Percentile (5\%) & & & \\
\hline 4 & Upper Percentile (95\%) & & & \\
\hline 5 & Standard Error & & & \\
\hline 6 & t-value & & & \\
\hline 7 & & 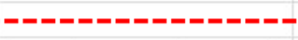 & 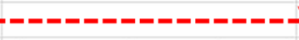 & 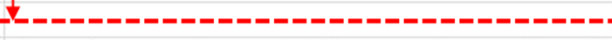 \\
\hline 8 & Formula & $(p 1+(p 5 \cdot S D$ of -1.0012$))$ & $((p 7 \cdot S D$ of -1.0012$)+p 2)$ & {$\left[(p 1+(p 5 \cdot S D \text { of }-1.0012))^{*}((p 7 \cdot S D\right.$ of -1.0012$\left.)+p 2)\right]$} \\
\hline 9 & & 0.260 & 0.325 & 0.085 \\
\hline 10 & & 0.309 & 0.378 & 0.117 \\
\hline 11 & & 0.343 & 0.403 & 0.138 \\
\hline 12 & & 0.291 & 0.487 & 0.142 \\
\hline 13 & & 0.273 & 0.347 & 0.095 \\
\hline
\end{tabular}

Figure D5. Step 5 Illustrative Example for Use of Continuous Moderator (CoMe Model C)

Create values for columns using Equation 13 in Table 1 (see main body in paper). This enables obtaining the subsample results for CoMe effect at medium moderator level (or Medium Sugro).

\begin{tabular}{|c|c|c|c|}
\hline$\triangle$ & Q & $\mathrm{R}$ & $S$ \\
\hline 1 & & & Medium Sugro \\
\hline 2 & & & \\
\hline 3 & & & \\
\hline 4 & & & \\
\hline 5 & & & \\
\hline 6 & & & \\
\hline 7 & & & Iا \\
\hline 8 & $(p 1+(p 5 \cdot S D$ of 0$))$ & $((p 7 \cdot S D$ of 0$)+p 2)$ & {$\left[(p 1+(p 5 \cdot S D \text { of } 0))^{*}((p 7 \cdot S D\right.$ of 0$\left.)+p 2)\right]$} \\
\hline 9 & 0.168 & 0.305 & 0.051 \\
\hline 10 & 0.263 & 0.349 & 0.092 \\
\hline 11 & 0.251 & 0.388 & 0.097 \\
\hline 12 & 0.229 & 0.406 & 0.093 \\
\hline 13 & 0.209 & 0.268 & 0.056 \\
\hline
\end{tabular}

Figure D6. Step 6 Illustrative Example for Use of Continuous Moderator (CoMe Model C) 
Create values for columns using Equation 14

in Table 1 (see main body in paper). This

enables obtaining the subsample results for

CoMe effect at high moderator level (or

High Sugro).

\begin{tabular}{|c|c|c|c|}
\hline$\triangle$ & $\mathrm{T}$ & u & V \\
\hline 1 & & & High Sugro \\
\hline 2 & & & \\
\hline 3 & & & \\
\hline 4 & & & \\
\hline 5 & & & \\
\hline 6 & & & \\
\hline 7 & - & 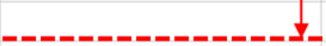 & 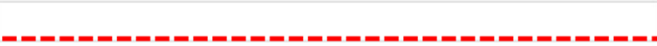 \\
\hline 8 & $(p 1+(p 5 \cdot S D$ of 1.0012$))$ & $((p 7 \cdot S D$ of 1.0012$)+p 2)$ & {$\left[(p 1+(p 5 \cdot S D \text { of } 1.0012))^{*}((p 7 \cdot S D\right.$ of 1.0012$\left.)+p 2)\right]$} \\
\hline 9 & 0.076 & 0.285 & 0.022 \\
\hline 10 & 0.217 & 0.320 & 0.069 \\
\hline 11 & 0.159 & 0.373 & 0.059 \\
\hline 12 & 0.167 & 0.325 & 0.054 \\
\hline 13 & 0.145 & 0.189 & 0.027 \\
\hline
\end{tabular}

Figure D7. Step 7 Illustrative Example for Use of Continuous Moderator (CoMe Model C)

6) Calculate the original values $(\mathrm{O})$ provided by the PLS-SEM path coefficients of each subsample (see Figure D8 to D10). The result shows that the path coefficient effects of the CoMe effects become weaker when the Sugro effect is one standard deviation above the mean. Subsequently, estimate the percentile bootstrap confidence interval $(\mathrm{Cl})$ using the function PERCENTILE (range, $\mathrm{k}$ ), where $\mathrm{k}$ is the percentile value between 0 and 1 (see Figure D8 to D10). If the $\mathrm{Cl}$ for the product "does not include a 0 value, this result means that the mediated effects at different levels of the moderator are significantly different from 0 ." In our example, the CoMe effects at low, medium, and high levels of the moderator are significant because both extremes of the $\mathrm{Cl}$ are positive. Therefore, we find some evidence to support the hypothesis of CoMe Model C; that is, the mediated effect of the consumer's PI on BI via PV reduces as SUGRO increases. 
Calculate the original value $(\mathrm{O})$ provided by PLS for path coefficients for CoMe effect at low moderator level (or Low Sugro). The detail is:

$=A V E R A G E(P 9: P 10008)$

\begin{tabular}{|c|c|c|c|c|}
\hline$\Delta$ & M & $\mathrm{N}$ & 0 & $\stackrel{p}{P}$ \\
\hline 1 & & & & Low \$ugro \\
\hline 2 & Original (O) & & 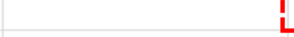 & 0.112 \\
\hline 3 & Lower Percentile (5\%) & & 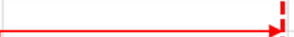 & 0.073 \\
\hline 4 & Upper Percentile (95\%) & & & 0.157 \\
\hline 5 & Standard Error & & & 0.026 \\
\hline 6 & t-value & & & 4.378 \\
\hline \multicolumn{5}{|l|}{7} \\
\hline 8 & Formula & $(\mathrm{p} 1+(\mathrm{p} 5 \cdot \mathrm{SD}$ of -1.0012$))$ & $((p 7 \cdot S D$ of -1.0012$)+p 2)$ & {$\left[(p 1+(p 5 \cdot S D \text { of }-1.0012))^{*}((p 7 \cdot S D\right.$ of -1.0012$\left.)+p 2)\right]$} \\
\hline 9 & & 0.260 & 0.325 & 0.085 \\
\hline 10 & & 0.309 & 0.378 & 0.117 \\
\hline 11 & & 0.343 & 0.403 & 0.138 \\
\hline 12 & & 0.291 & 0.487 & 0.142 \\
\hline 13 & & 0.273 & 0.347 & 0.095 \\
\hline
\end{tabular}

Calculate both the lower percentile (5\%) and the upper percentile (95\%).

- The details of the lower percentile for CoMe effect at low moderator level (or Low Sugro) is : $=$ PERCENTILE(P9:P10008, 0.05)

- The details of the upper percentile for CoMe effect at low moderator level (or Low Sugro) is: $=$ PERCENTILE(P9:P10008, 0.95)

Figure D8: Step 8 Illustrative Example for Use of Continuous Moderator (CoMe Model C) 
Calculate the original value $(\mathrm{O})$ provided by PLS for path coefficients for CoMe effect at medium moderator level (or Medium Sugro). The detail is:

$=$ AVERAGE(S9:S10008)

\begin{tabular}{|c|c|c|c|}
\hline$\triangle$ & Q & $\mathrm{R}$ & $\mathrm{s}$ \\
\hline 1 & & & Medium Sugro \\
\hline 2 & & & 0.075 \\
\hline 3 & & & 0.047 \\
\hline 4 & & & 0.107 \\
\hline 5 & & & 0.019 \\
\hline 6 & & & 4.035 \\
\hline \multicolumn{4}{|l|}{7} \\
\hline 8 & $(p 1+(p 5 \cdot S D$ of 0$))$ & $((p 7 \cdot S D$ of 0$)+p 2)$ & {$[(p 1+(p 5 \cdot S D$ of 0$)) *((p 7 \cdot S D$ of 0$)+p 2)]$} \\
\hline 9 & 0.168 & 0.305 & 0.051 \\
\hline 10 & 0.263 & 0.349 & 0.092 \\
\hline 11 & 0.251 & 0.388 & 0.097 \\
\hline 12 & 0.229 & 0.406 & 0.093 \\
\hline 13 & 0.209 & 0.268 & 0.056 \\
\hline
\end{tabular}

Calculate both the lower percentile (5\%) and the upper percentile (95\%).

- The details of the lower percentile for CoMe effect at medium moderator level (or Medium Sugro) is : =PERCENTILE(S9:S10008, 0.05)

- The details of the upper percentile for CoMe effect at medium moderator level (or Medium Sugro) is: =PERCENTILE(S9:S10008, 0.95)

\section{Figure D9: Step 9 Illustrative Example for Use of Continuous Moderator (CoMe Model C)}

Calculate the original value (O) provided by PLS for path coefficients for CoMe effect at high moderator level (or High Sugro). The detail is:

=AVERAGE(V9:V10008)

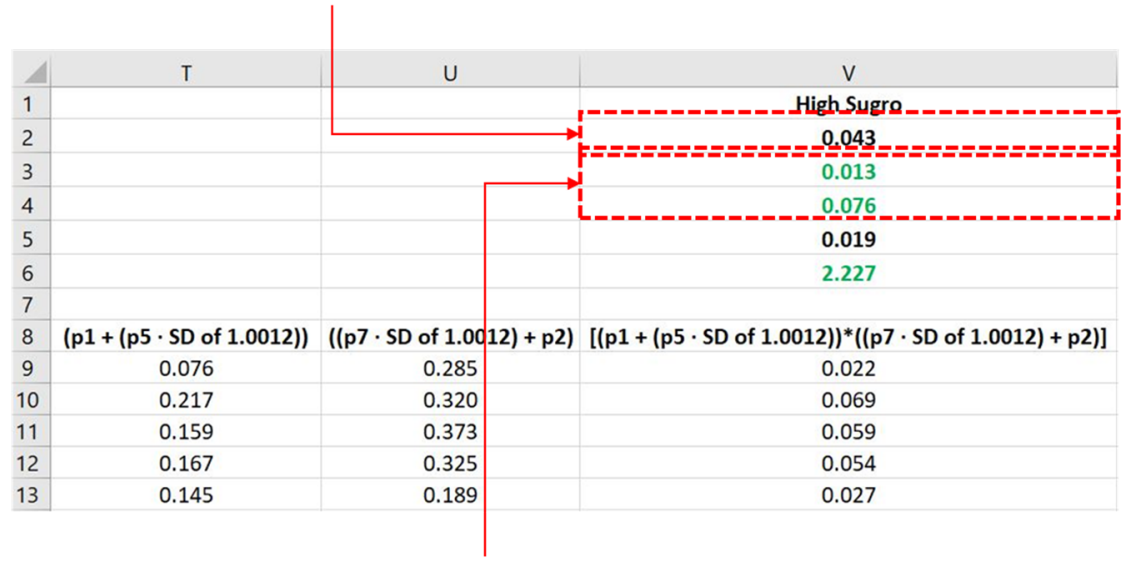

Calculate both the lower percentile (5\%) and the upper percentile (95\%).

- The details of the lower percentile for CoMe effect at high moderator level (or High Sugro) is : =PERCENTILE(V9:V10008, 0.05)

- The details of the upper percentile for CoMe effect at high moderator level (or High Sugro) is: =PERCENTILE(V9:V10008, 0.95)

Figure D10. Step 10 Illustrative Example for Use of Continuous Moderator (CoMe Model C) 
7) Finally, the researcher can create a graphical plot of the mediated effects at different levels of the moderator by inserting the estimated values in Figure D8 to D10 to an Excel spreadsheet, similar to the earlier suggestion in Figure B6 (see Figure 14 in the main body of paper).

\section{Appendix D(ii). Step-by-Step Procedure for Conditional Mediation Analysis (CoMe Model C) When Moderator Is Converted from Continuous to Categorical}

Given that the index of " $p_{2} \cdot p_{5}$ " can be obtained except for " $p_{1} \cdot p_{7}$ ", we decided to examine the results of the path coefficients using the output of the bootstrapping sample. Consequently, once we estimate the model, we next perform a bootstrapping procedure with 10,000 subsamples. Then, using a spreadsheet and multiplying the bootstrapping outputs, we calculate the percentile bootstrap $\mathrm{Cl}$ for the index of " $p_{2} \cdot p_{5}$ " and " $p_{1} \cdot p_{7}$ ". Subsequently, we estimate the index of CoMe Model $C$ by estimating the sum of both index of " $p_{2} \cdot p_{5}$ " and " $p_{1} \cdot p_{7}$ ". Thus, using an Excel spreadsheet, we suggest carrying out the following steps:

1) Take the 10,000 path coefficients from all direct effects created by the bootstrap procedure and copy and paste them into a spreadsheet (Figure D11).

\begin{tabular}{|c|c|c|c|c|c|c|c|c|}
\hline$\Delta$ & A & B & C & D & $\mathrm{E}$ & $\mathrm{F}$ & G & $\mathrm{H}$ \\
\hline 1 & Original (0) & & & & & & & \\
\hline 2 & Lower Percentile (5\%) & & & & & & & \\
\hline 3 & Upper Percentile (95\%) & & & & & & & \\
\hline 4 & Standard Error & & & & & & & \\
\hline 5 & t-value & & & & & & & \\
\hline 6 & & & & & & & & \\
\hline 7 & & p1 & p2 & p3 & p4 & p5 & p6 & p7 \\
\hline 8 & & $\mathrm{PI}>\mathrm{PV}$ & PV $\rightarrow>B I$ & $\mathrm{PI} \rightarrow \mathrm{BI}$ & Sugro $->$ PV & PI*Sugro $\rightarrow$ PV & Sugro -> BI & PV*Sugro -> BI \\
\hline 9 & Sample 0 & 0.425 & 0.356 & 0.316 & 0.297 & -0.094 & 0.157 & -0.139 \\
\hline 10 & Sample 1 & 0.358 & 0.448 & 0.276 & 0.388 & -0.229 & 0.137 & -0.117 \\
\hline 11 & Sample 2 & 0.323 & 0.4 & 0.177 & 0.337 & -0.127 & 0.219 & -0.125 \\
\hline 12 & Sample 3 & 0.377 & 0.43 & 0.283 & 0.391 & -0.119 & 0.124 & -0.1 \\
\hline 13 & Sample 4 & 0.335 & 0.45 & 0.257 & 0.372 & -0.094 & 0.118 & -0.1 \\
\hline
\end{tabular}

Figure D11. Step 1 Illustrative Example for Use of Categorical Moderator (CoMe Model C)

2) Create a new column for both the index of " $p_{2} \cdot p_{5}$ ", " $p_{1} \cdot p_{7}$ ", and " $p_{2} \cdot p_{5}+p_{1} \cdot p_{7}$ " as well as calculate the product of the paths that form the index under assessment (see Figure D12). 
Calculate the original values (0) to obtain the index value of "p2 * $\mathrm{p} 5$ ", "p1 * p7", and "p2* p5 + p1* p7".

The detail are: =AVERAGE(19:I10008), =AVERAGE(J9:J10008), and $=$ AVERAGE(K9:K10008)

\begin{tabular}{|c|c|c|c|c|c|c|c|c|c|c|c|}
\hline 4 & A & B & c & D & E & $\mathrm{F}$ & G & H & 1 & 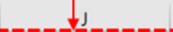 & $\underline{K}$ \\
\hline 1 & Original (0) & & & & & & & & -0.040 & -0.053 & -0.093 \\
\hline 2 & Lower Percentile (5\%) & & & & & & & & & & \\
\hline 3 & Upper Percentile (95\%) & & & & & & & & & & \\
\hline 5 & t-value & & & & & & & & -1.761 & -3.069 & -3.443 \\
\hline 6 & & & & & & & & & & & \\
\hline 7 & & p1 & p2 & p3 & p4 & p5 & p6 & p7 & p2*p5 & $\mathrm{p} 1^{*} \mathrm{p} 7$ & $p 2^{*} p 5+p 1^{*} p 7$ \\
\hline 10 & Sample 1 & 0.358 & 0.448 & 0.276 & 0.388 & -0.229 & 0.137 & -0.117 & -0.102 & -0.042 & -0.144 \\
\hline 11 & Sample 2 & 0.323 & 0.4 & 0.177 & 0.337 & -0.127 & 0.219 & -0.125 & -0.051 & -0.040 & -0.091 \\
\hline 12 & Sample 3 & 0.377 & 0.43 & 0.283 & 0.391 & -0.119 & 0.124 & -0.1 & -0.051 & -0.038 & -0.089 \\
\hline 13 & Sample 4 & 0.335 & 0.45 & 0.257 & 0.372 & -0.094 & 0.118 & -0.1 & -0.042 & -0.034 & -0.076 \\
\hline
\end{tabular}

Figure D12. Step 2 Illustrative Example for Use of Categorical Moderator (CoMe Model C)

3) Estimate the percentile bootstrap $\mathrm{Cl}$, particularly for columns " $p_{2} \cdot p_{5}+p_{1} \cdot p_{7}$ ", using the function PERCENTILE (range, $k$ ), where $k$ is the percentile value between 0 and 1 . In our case, given that our hypothesis postulates a direction, we use a one-sided test, and we estimate a $90 \% \mathrm{Cl}$ (Figure D13). If the $\mathrm{Cl}$ for the product of " $p_{2} \cdot p_{5}+p_{1} \cdot p_{7}$ " does not include a 0 value, this result means that the conditional mediating effect is significantly different from $0 .{ }^{9}$ In our example, the index " $p_{2} \cdot p_{5}+p_{1} \cdot p_{7}$ " is significant because both extremes of the $\mathrm{Cl}$ have negative sign. Therefore, we do find evidence to support the hypothesis of CoMe Model $\mathrm{C}$; that is, the mediated effect of a consumer's $\mathrm{PI}$ on $\mathrm{BI}$ via $\mathrm{PV}$ reduces as SUGRO increases.

Lower Percentile (5\%) for "p2 * p5" =PERCENTILE(I9:I10008;0,05)

Upper Percentile (95\%) for "p2 * p5" =PERCENTILE(I9:I10009; 0,95)

Lower Percentile (5\%) for "p1 * p7" =PERCENTILE(J9:J10008; 0,05)

Upper Percentile (95\%) for "p1 * p7" =PERCENTILE(J9:J10008; 0,95)

Lower Percentile (5\%) for “p2 * p5 + p1 * p7" =PERCENTILE(K9:K10008; 0,05)

Upper Percentile (95\%) for “p1* p5 + p1 * p7" =PERCENTILE(K9:K10008; 0,95)

\begin{tabular}{|c|c|c|c|c|c|c|c|c|c|c|c|}
\hline 4 & A & B & C & D & $E$ & $F$ & G & $\mathrm{H}$ & 1 & 1 & K \\
\hline 1 & Original (0) & & & & & & & & $=-0.040$ & $----\frac{6}{6} 053$ & -0.093 \\
\hline 2 & Lower Percentile (5\%) & & & & & & & & -0.078 & -0.084 & -0.137 \\
\hline 3 & Upper Percentile (95\%) & & & & & & & & -0.004 & -0.026 & -0.048 \\
\hline 4 & Standard Error & & & & & & & & $0 . \overline{02} \overline{3}$ & 0.017 & $0 . \overline{027}$ \\
\hline 5 & t-value & & & & & & & & -1.761 & -3.069 & -3.443 \\
\hline 6 & & & & & & & & & & & \\
\hline 7 & & p1 & p2 & p3 & p4 & p5 & p6 & p7 & $\mathrm{p} 2 * \mathrm{p} 5$ & $\mathrm{p} 1^{*} \mathrm{p} 7$ & $p 2 * p 5+p 1 * p 7$ \\
\hline 8 & & $\mathrm{PI} \rightarrow \mathrm{PV}$ & $\mathrm{PV} \rightarrow \mathrm{BI}$ & $\mathrm{PI} \rightarrow \mathrm{BI}$ & I Sugro -> PV & PI*Sugro -> PV & Sugro -> BI & PV*Sugro $->$ BI & PI*Sugro $\rightarrow$ PV $\rightarrow$ BI & PI*PV*Sugro -> BI & Index \\
\hline 9 & Sample 0 & 0.425 & 0.356 & 0.316 & 0.297 & -0.094 & 0.157 & -0.139 & -0.033 & -0.059 & -0.092 \\
\hline 10 & Sample 1 & 0.358 & 0.448 & 0.276 & 0.388 & -0.229 & 0.137 & -0.117 & -0.102 & -0.042 & -0.144 \\
\hline 11 & Sample 2 & 0.323 & 0.4 & 0.177 & 0.337 & -0.127 & 0.219 & -0.125 & -0.051 & -0.040 & -0.091 \\
\hline 12 & Sample 3 & 0.377 & 0.43 & 0.283 & 0.391 & -0.119 & 0.124 & -0.1 & -0.051 & -0.038 & -0.089 \\
\hline 13 & Sample 4 & 0.335 & 0.45 & 0.257 & 0.372 & -0.094 & 0.118 & -0.1 & -0.042 & -0.034 & -0.076 \\
\hline
\end{tabular}

Figure D13. Step 3 Illustrative Example for Use of Categorical Moderator (CoMe Model C)

9 To calculate the standard error and t-value, researchers can consider comments in Footnote 1 and Footnote 2 in this appendix. 
4) Create a new column for CoMe effects at low (or Low Sugro) and CoMe effects at high (or High Sugro) levels of moderator using the illustrated formula suggested in Figure D14.

Create values for these columns using Model C's

equation of the CoMe effects. This enables obtaining subsample results for CoMe effect at low moderator level (or Low Sugro) and CoMe effect at high moderator level (or High Sugro) by inserting the value of 0 and 1 respectively.

\begin{tabular}{|c|c|c|c|c|c|c|c|}
\hline 4 & M & $\mathrm{N}$ & 0 & $\mathrm{P}$ & Q & $\mathrm{R}$ & $s$ \\
\hline 1 & Original (0) & & & & & & \\
\hline 2 & Lower Percentile (5\%) & & & & & & \\
\hline 3 & Upper Percentile (95\%) & & & & & & \\
\hline 4 & Standard Error & & & & & & \\
\hline 5 & t-value & & & & & & \\
\hline 6 & & rーーーーーーーーー & 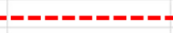 & 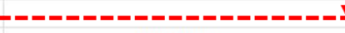 & 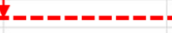 & 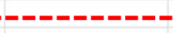 & 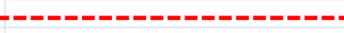 \\
\hline 7 & & & & Low Sugro & & & High Sugro \\
\hline 8 & Formula & $(p 1+(p 5 \cdot S D$ of 0$))$ & $((p 7 \cdot S D$ of 0$)+p 2)$ & {$\left[(p 1+(p 5 \cdot S D \text { of } 0))^{*}((p 7 \cdot S D\right.$ of 0$\left.)+p 2)\right]$} & $(p 1+(p 5 \cdot S D$ of 1$))$ & $((p 7 \cdot S D$ of 1$)+p 2)$ & {$\left[(p 1+(p 5 \cdot S D \text { of } 1))^{*}((p 7 \cdot S D\right.$ of 1$\left.)+p 2)\right]$} \\
\hline 9 & & 0.425 & $5 \quad 0.356$ & 0.151 & 0.331 & 0.217 & 0.072 \\
\hline 10 & & 0.358 & 0.448 & 0.160 & 0.129 & 0.331 & 0.043 \\
\hline 11 & & 0.323 & 0.400 & 0.129 & 0.196 & 0.275 & 0.054 \\
\hline 12 & & 0.377 & 0.430 & 0.162 & 0.258 & 0.330 & 0.085 \\
\hline 13 & & 0.335 & -0.450 & 0.151 & 0.241 & - 0.350 & 0.084 \\
\hline
\end{tabular}

Figure D14. Step 4 Illustrative Example for Use of Categorical Moderator (CoMe Model C)

5) Calculate the original values $(O)$ provided by the PLS-SEM path coefficients for each subsample (see Figure D15). The results show that the path coefficient effects of the CoMe effects become weaker for high Sugro than for low Sugro. Subsequently, estimate the percentile bootstrap confidence interval $(\mathrm{Cl})$ using the function PERCENTILE (range, $k$ ), where $k$ is the percentile value between 0 and 1 (see Figure D15). If the $\mathrm{Cl}$ for the product "does not include a 0 value, this result means that the mediated effects at different levels of the moderator are significantly different from 0 ." In our example, the CoMe effects for low Sugro and high Sugro are significant because both extremes of the $\mathrm{Cl}$ are positive. Therefore, we find some evidence to support the hypothesis of Model C; that is, the mediated effect of the consumer's PI on BI via PV reduces as SUGRO increases. 
Calculate the original value (O) provided by PLS for path coefficients for CoMe effect at low moderator level (or Low Sugro) and CoMe effect at high moderator level (or High Sugro). The detail are:

$=A V E R A G E(P 9: P 10008)$ and = AVERAGE(S9:S10008)

\begin{tabular}{|c|c|c|c|c|c|c|c|}
\hline$\Delta$ & M & $\mathrm{N}$ & 0 & P. & Q & $\underline{R}_{-}$ & -1 \\
\hline 1 & Original (0) & & 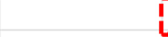 & 0.146 & & & 0.067 \\
\hline 2 & Lower Percentile (5\%) & & & 0.106 & & & 0.031 \\
\hline 3 & Upper Percentile (95\%) & & 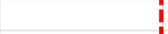 & 0.189 & & & 0.111 \\
\hline 4 & Standard Error & & & $-0.026=--=---0$ & $=-\pi$ & --- & 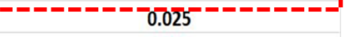 \\
\hline 5 & t-value & & & 5.658 & & & 2.699 \\
\hline \multicolumn{8}{|l|}{6} \\
\hline 7 & & & & Low Sugro & & & High Sugro \\
\hline 8 & Formula & $(p 1+(p 5 \cdot S D$ of 0$))$ & $((p 7 \cdot S D$ of 0$)+p 2)$ & {$\left[(p 1+(p 5 \cdot S D \text { of } 0))^{*}((p 7 \cdot S D\right.$ of 0$\left.)+p 2)\right]$} & $(p 1+(p 5 \cdot S D$ of 1$))$ & $((p 7 \cdot S D$ of 1$)+p 2)$ & {$\left[(p 1+(p 5 \cdot S D \text { of } 1))^{*}((p 7 \cdot S D\right.$ of 1$\left.)+p 2)\right]$} \\
\hline 9 & & 0.425 & 0.356 & 0.151 & 0.331 & 0.217 & 0.072 \\
\hline 10 & & 0.358 & 0.448 & 0.160 & 0.129 & 0.331 & 0.043 \\
\hline 11 & & 0.323 & 0.400 & 0.129 & 0.196 & 0.275 & 0.054 \\
\hline 12 & & 0.377 & 0.430 & 0.162 & 0.258 & 0.330 & 0.085 \\
\hline 13 & & 0.335 & 0.450 & 0.151 & 0.241 & 0.350 & 0.084 \\
\hline
\end{tabular}

Calculate both the lower percentile (5\%) and the upper percentile (95\%).

- The details of the lower percentile for CoMe effect at low moderator level (or Low Sugro) and CoMe effect at high moderator level (or High Sugro) are: =PERCENTILE(P9:P10008, 0.05) and =PERCENTILE(S9:S10008, 0.05)

- The details of the upper percentile for CoMe effect at low moderator level (or Low Sugro) and CoMe effect at high moderator level (or High Sugro) are: =PERCENTILE(P9:P10008, 0.95) and =PERCENTILE(S9:S10008, 0.95)

\section{Figure D15. Step 5 Illustrative Example for Use of Categorical Moderator (CoMe Model C)}

6) Finally, the researcher can create a graphical plot of the mediated effects at different levels of the moderator via an Excel spreadsheet (see Figure 14 in the main body of paper). 


\section{Appendix E. Result of Measurement Invariance Test using MICOM}

\begin{tabular}{|c|c|c|c|c|c|c|c|c|c|}
\hline \multirow[b]{2}{*}{$\underset{t}{\text { Construc }}$} & \multirow[b]{2}{*}{$\begin{array}{c}\text { Configural } \\
\text { Invariance } \\
\text { Establishe } \\
\text { d }\end{array}$} & \multicolumn{2}{|c|}{$\begin{array}{l}\text { Compositional } \\
\text { Invariance }\end{array}$} & \multirow[b]{2}{*}{$\begin{array}{c}\text { Partial } \\
\text { Measure } \\
\text { ment } \\
\text { Invarianc } \\
e \\
\text { Establis } \\
\text { hed }\end{array}$} & \multicolumn{2}{|c|}{ Equal Mean } & \multicolumn{2}{|c|}{ Equal Variance } & \multirow[b]{2}{*}{$\begin{array}{c}\text { Full } \\
\text { measureme } \\
\text { nt } \\
\text { invariance } \\
\text { established }\end{array}$} \\
\hline & & $c=1$ & $\begin{array}{c}\text { Confidence } \\
\text { Interval } \\
\text { (Cls) }-95 \%\end{array}$ & & $\begin{array}{l}\text { Difference } \\
\quad s\end{array}$ & $\begin{array}{c}\text { Confidence } \\
\text { Interval } \\
\text { (Cls) }-95 \%\end{array}$ & $\begin{array}{l}\text { Difference } \\
\quad s\end{array}$ & $\begin{array}{c}\text { Confidence } \\
\text { Interval } \\
\text { (Cls) }-95 \%\end{array}$ & \\
\hline $\mathrm{BI}$ & Yes & $\begin{array}{c}0.99 \\
4\end{array}$ & $\begin{array}{l}{[0.974} \\
1.000]\end{array}$ & Yes & 0.831 & $\begin{array}{c}-0.176 ; \\
0.156]\end{array}$ & 0.551 & $\begin{array}{c}{[-0.304} \\
0.299]\end{array}$ & No \\
\hline PV & Yes & $\begin{array}{c}1.00 \\
0\end{array}$ & $\begin{array}{l}{[0.999} \\
1.000]\end{array}$ & Yes & 0.863 & $\begin{array}{c}{[-0.164 ;} \\
0.163]\end{array}$ & 0.491 & $\begin{array}{c}{[-0.289} \\
0.285]\end{array}$ & No \\
\hline PI & Yes & $\begin{array}{c}0.99 \\
8\end{array}$ & $\begin{array}{r}{[0.990} \\
1.000]\end{array}$ & Yes & 0.671 & $\begin{array}{c}{[-0.161} \\
0.161]\end{array}$ & 0.592 & $\begin{array}{c}{[-0.277} \\
0.281]\end{array}$ & No \\
\hline
\end{tabular}

Note: $\mathrm{BI}=$ Behavioural Intention, $\mathrm{PV}=$ Perceived Value; and $\mathrm{PI}=$ Price Image

Based on Appendix $\mathrm{E}$, the results show that all composite constructs have significant differences in terms of the composite mean values and variances ratio because the results fall outside the upper and lower bounds of $95 \%$ confidence interval. Partial measurement invariance is thus established for the female and male groups (see last column in above table). It can be surmised that the different model estimations for the female and male groups are not distinct in terms of content or meaning of the constructs. 